Aus der Abteilung Allgemeinmedizin

(Prof. Dr. med. M. M. Kochen, MPH, FRCGP)

im Zentrum Innere Medizin

der Medizinischen Fakultät der Universität Göttingen

\title{
Der Übergang zwischen Selbstmedikation und ärztlicher Pharmakotherapie
}

Befragung von Patienten und ihren Hausärzten

\author{
INAUGURAL - DISSERTATION \\ zur Erlangung des Doktorgrades \\ der Medizinischen Fakultät \\ der Georg-August-Universität zu Göttingen \\ vorgelegt von \\ Ines Sajogo \\ aus \\ Haltern
}

Göttingen 2004 
D e k a n: $\quad$ Prof. Dr. med. M. Droese

I. Berichterstatter: Priv.-Doz. Dr. disc. pol. W. Himmel

II. Berichterstatterlin: Prof. Dr. Nicole von Steinbüchel

III. Berichterstatter/in:

Tag der mündlichen Prüfung: 14. Juni 2005 
1 Einleitung 1

2 Stand der Forschung 2

2.1 Definitionen 2

2.2 Anwendbarkeit rezeptfreier Arzneimittel 3

2.3 Geschichte der Selbstmedikation 4

2.4 Epidemiologie und Ökonomie 5

$\begin{array}{ll}2.4 .2 & \text { Internationaler Vergleich }\end{array}$

$\begin{array}{lll}2.4 .3 & \text { Indikationsbereiche } & 7\end{array}$

2.5 Gründe für die Selbstmedikation 9

2.6 Zufriedenheit mit und Wirksamkeit von Selbstmedikation 11

2.7 Gefahren der Selbstmedikation 11

$\begin{array}{ll}2.7 .1 \text { Nebenwirkungen } & 13\end{array}$

$\begin{array}{lll}2.7 .2 & \text { Interaktionen } & 16\end{array}$

$\begin{array}{lll}2.7 .3 & \text { Missbrauch } & 17\end{array}$

$\begin{array}{lll}2.8 & \text { Selbstmedikation und Hausarzt-Patient-Beziehung } & 17\end{array}$

3 Fragestellung und Hypothesen $\quad 19$

4 Methoden $\quad 21$

4.1 Auswahl der Arztpraxen $\quad 21$

$\begin{array}{lll}4.2 & \text { Patienten-Fragebogen } & 21\end{array}$

4.3 Arzt-Fragebogen $\quad 22$

$\begin{array}{lll}4.4 & \text { Pilotstudie } & 22\end{array}$

4.5 Auswahl der Patienten und Ablauf der Befragung 23

4.6 Datenanalyse 24

4.7 Medikamenten-Interaktionen 25

4.8 Datenschutz und Ethik 25

5 Ergebnisse $\quad 26$

$\begin{array}{lll}5.1 & \text { Patienten } & 26\end{array}$

5.2 Einnahmeverhalten der Patienten 28 
5.2.1 Verschriebene Medikamente 30

$\begin{array}{lll}\text { 5.2.2 Selbstmedikation } & 31\end{array}$

5.2.3 Zusammenhang zwischen der Anzahl an Dauermedikamenten und der Bereitschaft zur Einnahme von OTC-Präparaten

5.2.4 Einnahme von Selbstmedikation und Einnahme anderer Medikamente

5.2.5 Einnahme von Selbstmedikation, das Krankheitsbild und die Häufigkeit der Arztbesuche

5.3 Kommunikation mit dem Hausarzt über Selbstmedikation

5.4 Gründe und Themen der Unterhaltung über Selbstmedikation

5.5 Empfehlung zur Selbstmedikation durch den Hausarzt aus Patientensicht

5.6 Mögliche Interaktionen zwischen Selbstmedikation und verschriebener Medikation

5.7 Ärzte

5.8 Einschätzung der Selbstmedikation durch Hausärzte

5.9 Wichtigkeit der Kenntnis über das Selbstmedikationsverhalten der Patienten

5.10 Die Bereitschaft der Patienten, von ihrer Selbstmedikation zu berichten - aus Sicht der Hausärzte

5.11 Einstellung des Hausarztes zur Selbstmedikation und Kommunikation mit den Patienten

5.12 Empfehlung von Selbstmedikation durch den Hausarzt - aus Sicht der Hausärzte

\section{Diskussion}

6.1 Diskussion der Methode

6.2 Patienten-Stichprobe

6.3 Ärzte-Stichprobe 58

6.4 Einnahmeverhalten der Patienten

6.4.1 Verschriebene Medikamente 58

6.4.2 Selbstmedikation 
6.5 Anzahl Dauermedikamente und Bereitschaft zur Einnahme von OTC-

$\begin{array}{ll}\text { Präparaten } & 60\end{array}$

6.6 Selbstmedikation und Einnahme anderer Medikamente 61

6.7 Einnahme von Selbstmedikation, das Krankheitsbild und die Häufigkeit der Arztbesuche

6.8 Gespräch über Selbstmedikation mit dem Hausarzt

6.9 Empfehlung zur Selbstmedikation durch den Hausarzt

6.10 Interaktionen zwischen Selbstmedikation und verschriebener Medikation

6.11 Hausärztliche Beurteilung der Selbstmedikation

6.12 Einstellung des Hausarztes zur Selbstmedikation und Kommunikation mit den Patienten

7 Schlussfolgerungen

8 Zusammenfassung 73

9 Literatur 75

10 Anhang 85

10.1 Patienten-Fragebogen 85

10.2 Arzt-Fragebogen 91

10.3 Gesamtauszählung Patienten, N (\%) 94

10.4 Aushang 98

10.5 Informed consent 99

10.6 Arzneimittelgesetz 100

10.7 Aus der Rezeptpflicht entlassene Medikamente 101

10.8 Altersverteilung der teilnehmenden Patienten 


\section{Einleitung}

Patientenautonomie ist zu einem Leitbild moderner Medizin geworden. Selbstmedikation könnte ein wichtiges Element für eine Stärkung der Patientenautonomie sein (Bradley und Bond 1995; Forschungsgruppe Wahlen Online 2004; Himmel et al. 2003; Morris et al. 2001). Gleichzeitig gilt Kommunikation als ein Schlüsselelement in der Arzt-Patient-Beziehung. Damit stehen Ärzte, vor allem Hausärzte, vor einem Widerspruch oder einer schwierigen Frage: Fühlen sich Patienten durch die Frage des Hausarztes nach Selbstmedikation in ihren Autonomiebestrebungen beeinträchtigt oder wünschen sie das Gespräch bzw. eine Beratung über Selbstmedikation? Dies ist ein Thema der vorliegenden Arbeit.

Ein möglicher Beratungswunsch zur Selbstmedikation signalisiert bereits eine begriffliche Unschärfe: Selbstmedikation ist nicht unbedingt Einnahme von Medikamenten

ohne Beratung oder Wissen des Arztes. Umgekehrt werden verordnete Medikamente nicht unbedingt exakt nach „ärztlicher Anweisung“ eingenommen: nicht selten passen Patienten die Einnahme und Dosierung ihrer Medikamente eigenen Kriterien an, manchmal mit, manchmal ohne Wissen des verordnenden Arztes (Barat et al. 2000; Barat et al. 2001; Ceaser und Wurtz 2000). Im Sinne einer Sozialpharmakologie ist es daher wünschenswert, die Grauzone zwischen verordneter Medikation und autonomer Selbstmedikation genauer aufzuklären. Hieraus könnten sich Hinweise auf die Gefahr möglicher Arzneimittelinteraktionen ergeben - ein zweites Thema der Arbeit.

Auch zu den Einstellungen von Ärzten zur Selbstmedikation (ihrer Patienten) ist so gut wie nichts bekannt (Himmel 2000). In einem explorativen Ansatz werden - als dritter Aspekt der Arbeit - Einstellungen (,attitudes“) von Hausärzten zur Selbstmedikation und ggf. „kritische Ereignisse“ im Zusammenhang mit Selbstmedikation erfragt. 


\section{Stand der Forschung}

Neben einigen aktuellen Daten zur Häufigkeit von Selbstmedikation werden in diesem Kapitel insbesondere Gründe und potenzielle Gefahren der Selbstmedikation beleuchtet. Die Selbstmedikation im Rahmen der hausärztlichen Versorgung verdient dabei besondere Beachtung. Eingeleitet wird das Kapitel mit einigen wichtigen Definitionen.

\subsection{Definitionen}

\section{Selbstmedikation}

„Unter Selbstmedikation versteht man die eigenverantwortliche Anwendung von rezeptfreien Arzneimitteln (...) ohne vorausgegangene ärztliche Verordnung oder erneute Verordnung zur Vorbeugung oder Behandlung von leichten Erkrankungen und Befindlichkeitsstörungen."

(Walluf-Blume 1997, S. 30)

\section{OTC}

"Over the counter“ („über den Tresen“) lautet die international gebräuchliche Bezeichnung für rezeptfreie Mittel. Diese umfassen sowohl Arzneimittel der Selbstmedikation als auch von einem Arzt verordnete, aber nicht-verschreibungspflichtige Arzneimittel.

\section{Arzneimittel}

Arzneimittel sind zu diagnostischen, therapeutischen und prophylaktischen Zwecken verwendete, aus natürlichen Grundstoffen oder synthetisch hergestellte und gegebenenfalls speziell zubereitete Wirksubstanzen sowie chirurgisches Nahtmaterial, Desinfektionsmittel, Diagnostika und verschiedene Hilfsmittel (...). Herstellung und Umfang sind geregelt im Arzneimittelgesetz und in der Apothekenbetriebsordnung. (...) (Pschyrembel 2002, S. 135) 


\section{Freiverkäufliche Arzneimittel}

Diese sind "durch die §§ 44, 45 AMG von der Apothekenpflicht ausgenommen. Sie dürfen in sonstigen Einzelhandelsgeschäften abgegeben werden. Allerdings muss (...) eine Person mit Sachkunde anwesend sein, um bei Bedarf Kunden beraten zu können."

(Walluf-Blume 1998, S. 31)

\section{Switch}

"Switch wird die „Entlassung“ eines Wirkstoffes aus der Verschreibungspflicht genannt. Eine solche Entscheidung bereitet ein Sachverständigenausschuss beim Bundesgesundheitsministerium (BMG) vor. Der Beschluss der Sachverständigen ist eine Empfehlung an das BMG. Diese wird durch Rechtsverordnung in geltendes Recht umgesetzt" (Stieve 1996, S. 22). Nach § 49 Abs.4, Nr.3 AMG kann ein Medikament aus der Verschreibungspflicht entlassen werden, wenn die Voraussetzungen des § 48 Abs. 2, Nr.1 AMG nicht erfüllt sind (Anhang 11.6, S. 100).

Diese Voraussetzungen lauten:

1. Das Medikament enthält Substanzen, die den Konsumenten trotz bestimmungsgemäßen Gebrauchs gefährden können;

2. das Medikament hat ein großes Potenzial, nicht bestimmungsgemäß eingenommen zu werden.

Das Nichtvorliegen dieser Voraussetzungen wird durch die o.g. Sachverständigen geprüft.

\subsection{Anwendbarkeit rezeptfreier Arzneimittel}

Mit rezeptfreien Arzneimitteln sollten nur solche Leiden selbst behandelt werden, die den Erkrankten gut bekannt sind und deren Verlauf sie voraussehen können. Dabei variiert der für die Behandlung angemessene Zeitraum. Er sollte jedoch drei bis sieben Tage nicht überschreiten (AESGP 1997). 
Nicht angemessen ist Selbstmedikation bei folgenden Umständen:

- andauernde Symptomatik

- Verschlechterung des Zustandes

- starke Schmerzen

- Einstufung von Symptomen als schwerwiegend

- erfolglose Anwendung eines oder mehrerer Arzneimittel

- Vermutung von Arzneimittel-Interaktionen

- gleichzeitige psychiatrische Probleme (Angst, Depression, Übererregbarkeit).

Besondere Vorsicht ist bei Risikogruppen (Schwangere, Kleinkinder) geboten. Selbstmedikation gibt lediglich die Möglichkeit, Symptome und Beschwerden zu verhindern oder zu behandeln. Nur der Arzt kann eine medizinische Diagnose stellen (AESGP 1997).

\subsection{Geschichte der Selbstmedikation}

Als älteste Form der Heilmittelanwendung fand die Selbstmedikation bereits vor der Herausbildung medizinischer Berufe Anwendung. Wichtige Etappen in der Geschichte der Selbstmedikation beschreibt u.a. Friedrich (1990).

Im 12. / 13. Jahrhundert vollzog sich in Europa die Trennung zwischen ärztlicher und pharmazeutischer Tätigkeit. Anfänglich wurden unterschiedliche Waren angeboten, bis im 16. Jahrhundert die Verlagerung in Richtung eines vorrangigen Arzneimittelhandels erfolgte. Schon in diesen frühen Zeiten war es Apothekern nicht gestattet, alle Medikamente ohne Verschreibung durch einen Arzt zu vertreiben. Abgabebeschränkungen betrafen vor allem stark wirksame Arzneimittel. Es blieb jedoch immer ein gewisses Repertoire an Mitteln, die der Selbstmedikation dienen konnten.

Im 16. Jahrhundert kam es zum ersten Mal zu Kompetenzstreitigkeiten zwischen Ärzten und Apothekern um die Verschreibungspflicht von Arzneimitteln. Im Sinne eines Kompromisses wurde daraufhin differenziert zwischen verschreibungspflichtigen Arzneimitteln und Mitteln zur Selbstmedikation. Apotheker bekamen das Recht, leicht wirkende Mittel zu verkaufen, wie beispielsweise Prophylaktika, Laxanzien und Expektoranzien. Den enormen Aufschwung der Selbstmedikation im 16. Jahrhundert 
spiegelt das Erscheinen sogenannter Volksbücher wider. Sie boten eine Vielzahl von Vorschlägen zur medikamentösen Therapie der verschiedensten Krankheiten, die sich an billigen und leicht zu beschaffenden Arzneimitteln orientierten. Ebenfalls enthielten sie Anleitungen zur Eigenherstellung von Medikamenten.

Zur Entwicklung einer pharmazeutischen Industrie in Deutschland kam es am Übergang des 18. zum 19. Jahrhundert. Hiermit erlebte auch die Herstellung von Geheimmitteln zur Selbstmedikation einen erheblichen Aufschwung. Neu war das Auftreten sogenannter "wilder Apotheken“. Diese waren nicht von der Behörde genehmigte Apotheken, die einen selbstständigen Handel mit Arzneimitteln und Drogen betrieben. Aufgrund geringerer Unkosten konnten sie häufig die Preise der Apotheken unterbieten und nahmen auf diese Weise einen wichtigen Platz in der Selbstmedikation ein. Schließlich beteiligten sich auch die Drogerien am Verkauf eines ausgewählten Sortiments an Arzneimitteln (Friedrich 1990).

Fazit: Trotz schrittweise verlaufenden Verschärfungen der Medizinalgesetze blieb immer ein beachtlicher, wenn auch abnehmender Teil an Arzneimitteln für die Selbstmedikation erhalten. In den letzten Jahren ist eine Umkehr dieses Trends zu beobachten (s. unten).

\subsection{Epidemiologie und Ökonomie}

Die Selbstmedikation hat sich in den letzten Jahren - von zeitweiligen Schwankungen abgesehen - deutlich erhöht. Seit 1978 sind die Umsätze von 1,56 Mrd. Euro (nur Westdeutschland) bis 1998 auf 4,55 Mrd. Euro (Gesamtdeutschland; 3,89 Mrd. Euro nur Westdeutschland) gestiegen. 1999 fiel der Umsatz auf 4,2 Mrd. Euro. Seitdem stagniert der Umsatz (Tabelle 1). 1999 hatte der Selbstmedikationsmarkt einen Anteil von über 40\% am Gesamtabsatz der Arzneimittelpackungen (BAH 2000). Im Jahr 2000 gaben Patienten 8,2 Mrd. DM für Selbstmedikation aus gegenüber 5,8 Mrd. DM, die für Verordnungen von OTC-Präparaten ausgegeben wurden (BPI 2001). In den letzten zwei Jahren ist eine Stagnation bzw. 2003 sogar ein Rückgang der Selbstmedikation zu beobachten. 
Tabelle 1: $\quad$ Umsatz nicht verschriebener Pharmaka (Endverbraucherpreise)

\begin{tabular}{cc}
\multicolumn{2}{c}{ in Apotheken und außerhalb; ausgewählte Jahre } \\
\hline Jahr & in Mrd. Euro \\
\hline 1978 & 1,56 (West) \\
1984 & 2,23 (West) \\
1996 & 4,37 \\
1997 & 4,60 (West: 3,94) \\
1998 & 4,55 (West: 3,89) \\
1999 & 4,20 \\
2000 & 4,20 \\
2001 & 4,26 \\
2002 & 4,20 \\
\hline
\end{tabular}

(nach BAH 1997-2003, nach Winkelmann 1988, S. 80)

Während das rezeptfreie Marktsegment im Apothekenmarktumsatz von 2000 auf 2001 nur noch um 1,9\% wuchs (BPI 2002), fiel es 2002 um 1,86\% auf 3.456,6 Mio. Euro (BPI 2003).

\subsubsection{Internationaler Vergleich}

Im europäischen Vergleich liegt Deutschland mit Abstand an der Spitze des Selbstmedikationsmarktes vor Großbritannien. Am Ende der Liste liegt Slowenien, wobei hier sogar ein Rückgang der Selbstmedikation zu verzeichnen ist. Ebenfalls zurückgegangen ist der Selbstmedikationsmarkt in Frankreich und Polen. In Finnland stagniert der Selbstmedikationsmarkt. Beachtlich ist die Entwicklung der Zahlen in Kroatien und der Tschechischen Republik mit einer deutlichen Zunahme des Marktes (Tabelle 2). 
Tabelle 2: Europäischer Selbstmedikations-Markt 2001 bis 2003

\begin{tabular}{lccc}
\hline Land & $\begin{array}{c}\mathbf{2 0 0 1} \\
\text { (in Mio. Euro) }\end{array}$ & $\begin{array}{c}\mathbf{2 0 0 2} \\
\text { (in Mio. Euro) }\end{array}$ & $\begin{array}{c}\mathbf{2 0 0 3} \\
\text { (in Mio. Euro) }\end{array}$ \\
\hline Deutschland & 4269,0 & 4205,0 & 4257,0 \\
Großbritannien & 2774,4 & 2801,2 & 2850,9 \\
Frankreich & 1662,0 & 1549,0 & 1572,0 \\
Italien & 1293,0 & 1351,0 & 1510,0 \\
Spanien & 868,0 & 920,0 & 1073,0 \\
Slowakei & 803,5 & 838,8 & 984,5 \\
Polen & 771,0 & 681,0 & 675,0 \\
Schweiz & 653,1 & 655,1 & 629,1 \\
Niederlande & 510,1 & 519,8 & 530,2 \\
Belgien & 471,1 & 486,5 & 506,8 \\
Österreich & 244,5 & 253,0 & 273,6 \\
Tschechische Republik & 229,0 & 250,0 & 291,3 \\
Griechenland & 208,3 & 225,9 & 250,0 \\
Portugal & 204,1 & 234,7 & 251,3 \\
Schweden & 204,0 & 218,0 & 225,0 \\
Ungarn & 174,2 & 214,0 & 237,8 \\
Finnland & 171,0 & 169,0 & 175,0 \\
Irland & 170,0 & 197,3 & 215,3 \\
Dänemark & 117,6 & 125,3 & 131,1 \\
Rumänien & 101,2 & 110,5 & 105,3 \\
Norwegen & 86,7 & 99,1 & 96,3 \\
Kroatien & 42,7 & 58,5 & 66,7 \\
Slowenien & 28,3 & 27,8 & \\
\hline
\end{tabular}

(nach AESGP 2004)

\subsubsection{Indikationsbereiche}

Den höchsten Absatz im Selbstmedikationsmarkt haben mit einer Vorreiterposition Arzneimittel für Husten und Erkältung. Es folgen Mittel für die Verdauung und den Magen sowie Schmerzmittel. Vergleichsweise selten und mit abnehmender Tendenz werden Präparate für Beruhigung und Schlaf ohne Rezept erworben (Tabelle 3). 
Tabelle 3: Umsatzstärkste Indikationsbereiche der Selbstmedikation in Apotheken,

Drogerie- und Verbrauchermärkten in Deutschland 1998, 2000 - 2003

(Endverbraucherpreise)

\begin{tabular}{lccccc}
\hline Indikationsbereich & $\begin{array}{c}\mathbf{1 9 9 8} \\
\text { (in Mio. Euro) }\end{array}$ & $\begin{array}{c}\mathbf{2 0 0 0} \\
\text { (in Mio. Euro) }\end{array}$ & $\begin{array}{c}\mathbf{2 0 0 1} \\
\text { (in Mio. Euro) }\end{array}$ & $\begin{array}{c}\mathbf{2 0 0 2} \\
\text { (in Mio. Euro) }\end{array}$ & $\begin{array}{c}\mathbf{2 0 0 3} \\
\text { (in Mio. Euro) }\end{array}$ \\
\hline Husten und Erkältung & 789 & 853 & 866 & 839 & 944 \\
Magen und Verdauung & 529 & 550 & 559 & 553 & 553 \\
Schmerz & 465 & 474 & 476 & 471 & 481 \\
Vitamine und Mineralstoffe & 428 & 394 & 399 & 363 & 344 \\
Haut und Wundheilung & 316 & 316 & 335 & 333 & 340 \\
Tonika und Geriatrika & 299 & 277 & 260 & 274 & \\
Herz und Kreislauf & 282 & 316 & 338 & 334 & \\
Rheuma- und Muskelschmerz & 280 & 305 & 330 & 353 & \\
Beruhigung und Schlaf & 254 & 239 & 221 & 199 \\
Andere & 908 & 460 & 483 & 486 \\
\hline Gesamt & 4.549 & 4.184 & 4.267 & 4.205 & \\
\hline
\end{tabular}

(nach BAH 1999, 2001, 2002, 2003; nach AESGP 2004)

In den Jahren 1998 und 2000 gab es leichte Differenzen zwischen alten und neuen Bundesländern. In Deutschland-Ost wurden im Vergleich mehr Schmerzmittel erworben. Sie bildeten den zweitstärksten Umsatzbereich vor den Verdauungsmitteln. Auch der Umsatz an Tonika und Geriatrika sowie Herz- und Kreislaufmitteln lag vergleichsweise höher. Für 2001 wurden die Daten nicht mehr getrennt betrachtet. Auffallend ist die Zunahme der Ausgaben für Herz- und Kreislaufmittel um 52 Mio. Euro und die Abnahme der Umsätze für Vitamine und Mineralstoffe um 84 Mio. Euro, sowie im Bereich Tonika und Geriatrika (25 Mio. Euro) und Beruhigung und Schlaf (55 Mio. Euro). Das größte Wachstum zeigten Ausgaben für Husten- und Erkältungsmittel. Hier sprangen die Ausgaben seit 1998 um 77 Mio. Euro auf 866 Mio. Euro in 2001. 2002 erfahren alle Indikationsbereiche Einbußen. Ausgaben für Beruhigungsund Schlafmedikamente sinken mit 8\% gegenüber dem Vorjahr am stärksten. Lediglich Ausgaben für Tonika und Geriatrika steigen wieder an. Zahlen für das Jahr 2003 
liegen nur unvollständig vor. Ausgaben für Vitamine und Mineralstoffen fallen weiter $a b$.

In Europa zeigt sich eine ähnliche Verteilung. Führend sind auch hier Medikamente für „cough and cold“ (2424,95 Mio. Euro). Es folgen „analgesics“ (2966,3 Mio. Euro) und „digestives“ mit 2391,2 Mio. Euro. „Vitamins and minerals“ und „skintreatment“ liegen dahinter mit 2091,2 und 1808,5 Mio. Euro (AESGP 2002).

\subsection{Gründe für die Selbstmedikation}

Einer der möglichen Gründe für Selbstmedikation sind die in den achtziger Jahren eingeführten und seitdem immer wieder erhöhten Zuzahlungen für Arzneimittel. Eine Emnid-Umfrage, die 1998 vom Bundesverband für Pharmazeutische Industrie (BPI) vorgestellt wurde ergab, dass $41 \%$ aller Patienten erhöhte Zuzahlungen zum Anlass nahmen, zukünftig seltener zum Arzt zu gehen. 49\%, also fast die Hälfte, wollten Rezepte, die der Arzt verschreibt, nicht mehr einlösen und 29\% der Patienten gaben an, künftig noch stärker zur Selbstmedikation zu greifen (EMNID-Institut 1998).

Einen weiteren wichtigen Grund bietet das „neue Gesundheits-Bewusstsein“ der Bevölkerung. Einer Bevölkerungsumfrage zur Selbstmedikation zufolge, unternehmen 96\% der Befragten vorbeugend etwas zur Gesundheitserhaltung, vor allem durch weniger Zigaretten und Alkohol, gesündere Ernährung und mehr Bewegung. Arzneimittel zur Vorbeugung nahmen laut eigenen Angaben 53\% im Westen und 55\% im Osten Deutschlands. Befragt wurden 2.562 Personen in den Jahren 1990 und 1994 (I+G Infratest und GfK Gesundheits- und Pharmamarkt-Forschung o. J.). Eine kürzlich abgeschlossene Online-Befragung von 5.182 Personen ergab, dass viele Befragte sich gerne selber und ohne Arzt behandeln wollen. Auch Vorbeugung war für $62 \%$ der Befragten ein Grund für Selbstmedikation. 50\% nannten als Grund, dass rezeptfreie Medikamente nicht mehr von den Kassen übernommen würden (Forschungsgruppe Wahlen Online 2004).

Andere Gründe sind eher praktischer Natur. So nehmen viele Patienten gerne das Angebot an, auf eigene Initiative zur Apotheke zu gehen und auf diese Weise die Zeit und die Kosten des Arztbesuches einzusparen. Dies war Ergebnis eines Gespräches 
von Experten und Patienten zum Thema Selbstmedikation (Berg 1994). Auch Bradley und Bond (1995) führten diesen Punkt als Vorteil der Selbstmedikation an. Hassell et al. führten 1997 Interviews in Apotheken mit Fachpersonal und Kunden mit der Fragestellung, warum immer mehr Menschen bei Krankheit den Apotheker und nicht den Arzt als erste Anlaufstelle wählen. Aus den Kundeninterviews ging hervor, dass viele wegen geringer Gesundheitsprobleme (Erkältung, Husten, Halsschmerzen, Schmerzen) eher in die Apotheke als zum Hausarzt gingen. Als Gründe wurden genannt: Bequemlichkeit, Zeit- und Kostenersparnis, aber auch die Einstellung, den Hausarzt nicht wegen leichter Krankheiten zu belasten und Schwererkranken den Platz zu nehmen. Eine weitere Meinung war, dass der Apotheker als eine Art Filter agiere, der diejenigen zum Arzt schicke, die es nötig hätten, und den anderen zu einem wirksamen Medikament rate.

Weiterhin werden Gründe genannt, wie geringe Nebenwirkungen, eigene oder von Verwandten und Bekannten berichtete gute Erfahrungen, Steigerung der Leistungsfähigkeit oder einfach die Überzeugung „selbst am besten zu wissen, was einem hilft".

Diesen letztgenannten Aspekt unterstreicht das Ergebnis einer Studie des Pharmazeutischen Instituts der Universität Tübingen. Per Telefoninterview wurden medizinische Laien zu Arzneimitteln, deren Herstellung, Anwendungsgebiete und Wirkung befragt. Interviewt wurden Erwachsene zwischen 20 und 29 Jahren, sowie zwischen 60 und 69 Jahren in Aachen. Es konnte festgestellt werden, dass die befragten Personen gute Kenntnisse im Bereich Selbstmedikation hatten und auch sinnvolle Angaben zur sicheren Anwendung machen konnten (Anonym 1997). Auch die von Bradley und Bond (1995) zitierte Studie der Proprietary Association of Great Britain konnte zeigen, dass die Patienten im Großen und Ganzen verantwortungsvoll mit inrer Selbstmedikation umgingen. In $45 \%$ geringfügiger Leiden nahmen Patienten keine Medikation ein. Wurde mediziert, so geschah dies auf angebrachte Weise. In den meisten Fällen nahmen die Patienten nur ein Medikament zur gleichen Zeit ein.

Bessere Möglichkeiten für den Patienten mehr Eigenverantwortung für die eigene Gesundheit zu übernehmen (Bradley und Bond 1995) stellen ebenfalls positive Aspekte der Selbstmedikation dar. 


\subsection{Zufriedenheit mit und Wirksamkeit von Selbstmedikation}

Generell ist die Zufriedenheit in Deutschland mit Arzneimitteln zur Selbstmedikation groß. Nach einer Umfrage von 1994 äußerten 87\% im Westen und 82\% im Osten Deutschlands Zufriedenheit mit der Selbstmedikation (I+G Infratest und GfK Gesundheits- und Pharmamarkt-Forschung o.J.). Worauf diese Zufriedenheit bezogen ist, wurde nicht weiter charakterisiert. Studien zur subjektiv empfundenen Wirksamkeit der eingenommenen Mittel unterstützen die Ergebnisse. In der bereits erwähnten Studie der Universität Tübingen glaubten 97\% der Befragten an die Wirksamkeit des von ihnen eingenommenen Arzneimittels. In einer Umfrage des INRA-Instituts in Mölln von 1996 für die Zeitschrift Focus (Esser und Rehm 1996) gaben 82\% der Befragten eine Linderung ihrer Beschwerden an. Focus ließ 1000 Personen ab 14 Jahren in Deutschland befragen. Untersuchungen zur tatsächlichen Wirksamkeit, vor allem von Erkältungspräparaten, sind rar. Schroeder und Fahey (2002) veröffentlichten ein Review über randomisierte Placebo-kontrollierte Studien zur Wirksamkeit von OTC-Medikamenten gegen Erkältungskrankheiten. Nach intensiver Literaturrecherche erfüllten nur 15 Studien die Einschlusskriterien. In neun von fünfzehn Studien wurde kein Unterschied zwischen der Behandlung mit dem OTC-Präparat und dem Placebo festgestellt. Die Autoren warnen jedoch selber vor der Interpretation der Ergebnisse, da Methoden und Zielparameter sehr variierten und einen Vergleich so erschweren. Auch Smith und Feldman (1993) untersuchten Studien zur Wirksamkeit von OTC-Erkältungsmedikamenten im Zeitraum zwischen 1950 und 1991. Auch sie fanden zum Teil widersprüchliche Ergebnisse, kamen aber zu dem Schluss, dass viele Produkte Erleichterung verschaffen. Hierunter fielen auch Kombinationsprodukte.

\subsection{Gefahren der Selbstmedikation}

Trotz großer Beliebtheit bei den Konsumenten sehen Ärzte und Apotheker ein beträchtliches Gefahrenpotenzial in der Entwicklung der Selbstmedikation. Ihre Bedenken richten sich sowohl gegen freiverkäufliche als auch gegen apothekenpflichtige Arzneimittel. 
H. R. Vogel, Ehrenvorsitzender des BPI, befürchtet in Bezug auf die bereits erwähnte EMNID-Umfrage vom März 1998, dass viele Krankheiten durch das Auslassen oder Hinausschieben des Arztbesuches unbehandelt blieben bzw. zu spät behandelt würden. Die Krankheit könne sich dadurch verschlimmern oder in ein chronisches Stadium übergehen (Anonym 1998). Erwin et al. (1997) befassen sich mit den Bedenken von Hausärzten gegenüber Selbstmedikation in Bezug auf OTC-H2-Antagonisten: $24 \%$ der Befragten befürchteten einen Kontrollverlust über die Medikamenteneinnahme des Patienten; 45\% sehen eine Gefahr in durch OTC-Medikamente maskierten Symptomen oder versäumten Diagnosen. Fünf Jahre nachdem die $\mathrm{H}_{2}$ Rezeptorantagonisten Cimetidin und Ranitidin in Dänemark "geswitcht" wurden, untersuchte Andersen (1994) die Entwicklung um diese Medikamente. Besonders gefürchtet war hier das Nicht-Erkennen eines Magenkarzinoms durch Verschleierung der Frühsymptome. In den fünf Jahren nach der Reklassifikation wurden keine ernstzunehmenden Nebenwirkungen bekannt. Auch stieg die Anzahl an Krankenhauseinweisungen aufgrund von Komplikationen nicht an. Leider gibt Andersen keine Ergebnisse über die Veränderungen in Bezug auf das Magenkarzinom.

Dass ein Risiko nicht allein für OTC-Präparate besteht, konnte eine Studie von 1997 aufzeigen. Erwachsene zwischen 18 und 50 Jahren mit Asthma bronchiale wurden interviewt, um die Frequenz und das Risiko der Selbstbehandlung von Asthma zu erforschen. Erfragt wurde die Einnahme von pflanzlichen Mitteln, Kaffee und schwarzem Tee (zur Asthmatherapie) und OTC-Präparaten in den vergangenen 12 Monaten. Danach waren Kaffee und Tee stark mit einem steigenden Risiko der Notfallaufnahme und Hospitalisation assoziiert. Therapie mit pflanzlichen Mitteln stand mit einer erhöhten Anzahl von Krankenhausaufenthalten in Verbindung, während das Risiko der OTC-Anwender nicht erhöht war (Blanc et al. 1997).

Weiterhin berge Selbstmedikation nach Ansicht von Ärzten die Gefahr der Bagatellisierung ernsthafter Gesundheitsschäden (Baines und Whynes 1997). Bradley und Bond (1995) sehen vor allem ein Problem in Interaktionen, von denen besonders Ältere gefährdet seien. Darüber hinaus befürchten auch sie die Maskierung ernsthafter Krankheitssymptome, die Einnahme falscher Medikamente oder inkorrekte Dosierung. Gysling (1998, S. 66) gibt die möglichen Gefahren der Selbstmedikation wieder. Diese stimmen mit den Befürchtungen anderer Autoren überein: 
1. Die vermutete Diagnose ist falsch.

2. Die gewählte Medikation ist nicht die beste.

3. Die notwendige Behandlung wird verzögert.

4. Eine Symptombehandlung hält davon ab, schädliche Verhaltensweisen zu ändern.

5. Ein Konflikt mit einer anderen (verschriebenen) Arzneimitteltherapie ist möglich.

6. Auftreten unvermuteter Nebenwirkungen.

Bradley und Bond (1995) sowie Gysling (1998) warnen zudem vor einer Entwicklung, in der die Menschen an eine Pille gegen jedes Leiden glauben. Während Bradley und Bond vom Glauben an ein System mit der "pill for every ill“ (S. 554) sprechen, nennt es Gysling das „Konzept, mittels Selbstmedikations-Produkten [...] die Gesundheit wieder zu ,kaufen'“(S. 68).

\subsubsection{Nebenwirkungen}

Studien über unerwünschte Wirkungen bei Selbstmedikation befassen sich vor allem mit nicht steroidalen Antirheumatika (NSAR) und Analgetika, da der Schmerzmittelkonsum ausgesprochen hoch ist. In den letzten Jahren werden aber zusehends auch Daten zu anderen häufig verwendeten rezeptfreien Arzneimitteln erhoben:

\subsubsection{Nicht steroidale Antirheumatika}

Bereits 1960 wurden erste epidemiologische Studien zu OTC-Analgetika durchgeführt, nachdem es zu einer Epidemie an Nierenversagen in den westlichen Ländern gekommen war. Diese wurde schnell mit dem gleichzeitig rapiden Anstieg des OTCAnalgetika-Konsums in Verbindung gebracht, der damals um das Vierfache anstieg (Abbott und Fraser 1998). Alle Studien kamen zu dem Ergebnis, dass NSAR zumindest bei langfristiger Einnahme eine Reihe von Nebenwirkungen haben.

Die Inhibierung der Bildung von Prostaglandinen in der Magen- und Darmwand kann zu Entzündungen, Blutungen und Ulzerationen führen (Abbott und Fraser 1998). Auf diese Weise verursachte Leiden konnten in mehreren Studien auf Langzeittherapien mit den genannten Substanzen zurückgeführt werden. In der von Abbott und Fraser 1998 veröffentlichten Studie konnte gezeigt werden, dass die Langzeittherapie mit 
NSAR das Risiko einer gastrointestinalen Blutung um das 10- bis 30-fache erhöht. Die Effekte waren dosisabhängig, konnten aber schon bei Dosen zur Prophylaxe von kardiovaskulären Erkrankungen festgestellt werden. Zu anderen Ergebnissen kommt eine Studie von Doyle et al. (1999): Hier ergab die bestimmungsgemäße Einnahme eine gute Verträglichkeit. Durch NSAR verursachte renale Probleme sind Nierenversagen und die sogenannte Analgetika-Nephropathie (Abbott und Fraser 1998). Letztere beschreibt eine interstitielle Nephropathie infolge kontinuierlichen Missbrauchs peripher wirkender Analgetika.

Pommer et al. (1989) untersuchten das Auftreten der Analgetika-Nephropathie bei der Einnahme von Kombinationspräparaten. In die Studie aufgenommen wurden alle Patienten, die zwischen 1984 und 1986 in Westberlin chronische Dialyse betrieben. Das Risiko war deutlich erhöht bei Präparaten, die in einer Menge von 15 Dosierungen und mehr pro Monat für mindestens ein Jahr eingenommen wurden. Für Monopräparate galt dieser Zusammenhang nicht. Fox (1997) interpretiert die Ergebnisse anders. Er erkennt keinen Unterschied in der nephrotoxischen Wirkung zu den Monopräparaten. Weiterhin stellt er den genannten Ergebnissen Zahlen der European Dialysis and Transplantation Association gegenüber, die kein erhöhtes Nephropathie-Risiko zeigen.

Gründe für die Einnahme von Analgetika sind vor allem Kopfschmerz, Fiebererkrankungen und Rückenschmerzen. Frauen verwenden sie außerdem bei depressiven Stimmungen und prämenstruellen Beschwerden. Männer geben Schlafstörungen und verminderte physische Aktivität als weitere Gründe an (Abott und Fraser 1998). Weiter festzustellen war in allen Studien, dass der Schmerzmittelkonsum unter Frauen höher ist als unter Männern.

\subsubsection{Laxanzien}

Studien zu Wirkungen von Laxanzien konnten eine Reihe gastroenterologischer, kardialer, neurologischer, sowie renaler Nebenwirkungen aufzeigen. Bei regelmäßiger Einnahme könne es zum sogenannten Laxanziencolon kommen, was eine Funktionsstörung des Dickdarms beschreibt (Knopf et al. 1995). Weiter führe der Gebrauch zu Flatulenz, Übelkeit, Diarrhoe und Pruritus. Durch eine Hypermagnesiämie könne 
es zu Nierenfunktionsstörungen kommen, weiterhin zu einer Hypertonie. Chronische Einnahme führe zu zusätzlichen Elektrolytstoffwechselstörungen, insbesondere zu Hypokaliämien, die ihrerseits zu verstärkter Obstipation führten. So geriete der Patient in einen Teufelskreis, der zu ständig höheren Dosen Laxanzien führe (Wick et al. 1998).

Trotz dieser Studien sehen Hitzenberger und sein Expertenforum (1999) in der Therapie der Obstipation mit Laxanzien die beste Lösung. In Tierexperimenten zeigten Sennoside, Bisacodyl und Natriumpicosulfat unter chronischer Gabe keine spezifischen toxischen Effekte. Bei Sennosiden seien auch bei hohen Dosen keine Hypokaliämien aufgetreten. Anders läge der Fall bei Laxanzien-Abusern, die zusätzlich zu extremer Überdosierung (bis zum 100-fachen der Tagesdosis) häufig ihre Nahrungsaufnahme stark einschränkten oder Erbrechen induzierten. Hier könne es zu Hypokaliämien, sekundärem Hyperaldosteronismus und Gewöhnung, Herzrhythmusstörungen und Nierenversagen kommen.

\subsubsection{Phytopharmaka}

Studien über pflanzliche Arzneimittel und Heilkräuter konnten eine Reihe ernst zu nehmender Nebeneffekte aufzeigen.

Bei einigen dieser Produkte kam es zu hypertensiven und allergischen Reaktionen, die von kurzzeitigen Dermatitiden bis zum anaphylaktischen Schock reichten. Auch psychogene Wirkungen (Halluzinationen, Paranoia etc.) wurden beobachtet. Die in vielen Präparaten enthaltenen Flavonoide führten in manchen Fällen zu hämolytischen Anämien, weiterhin zu chronischen Diarrhoen, Colitiden und schweren Nephropathien. In Frankreich wurde Germander, ein Produkt zur Gewichtskontrolle, vom Markt genommen, nachdem in 30 Fällen ein akutes Nierenversagen nach der Einnahme auftrat. Pflanzliche Tees konnten mit akuter Hepatitis assoziiert werden. Ebenfalls wurden Präparate der chinesischen Medizin als lebertoxisch erkannt. Weiter wurden interstitielle renale Fibrosen und Nierenversagen beobachtet (Ernst 1998). Miller (1998) nennt Nebenwirkungen unter anderem bei Echinacea (hepatotoxische Effekte), Ingwer (Verlängerung der Blutungszeit durch Inhibierung der Thromboxan- 
Synthetase) und Ginkgo (gastrointestinale Beschwerden, Kopfschmerzen, spontane bilaterale subdurale Hämatome).

\subsubsection{Interaktionen}

Durch die ständig steigende Zahl an "geswitchten“ Medikamenten befassen sich immer mehr Studien mit der Problematik von Interaktionen zwischen OTCMedikamenten und verschriebenen Arzneimitteln. Sihvo et al. (2000) führten 1995 und 1996 computerassistierte Interviews zur Einnahme von OTC-Medikamenten in 5171 Haushalten (10477 Erwachsene ab 15 Jahren) durch. Mit Hilfe des Swedish Drug Compendium FASS erarbeiteten sie mögliche Interaktionen zwischen den eingenommenen OTC-Medikamenten und verschreibungspflichtigen Medikamenten. Sie fanden heraus, dass es in $4 \%$ potenziell zu Interaktionen zwischen OTC- und verschriebenen Medikamenten kommen könnte. Darunter waren vor allem die Kombinationen NSAR mit Betablockern oder Diuretika und ASS mit ACE-Hemmern. Der stärkste Prediktor für mögliche Interaktionen war die Anzahl an verschreibungspflichtigen Arzneimitteln.

Viele Studien widmen sich im Gegensatz zu Sihvo et al. speziellen MedikamentenKombinationen. So untersuchten Johne et al. 1999 in Berlin Interaktionen zwischen Extrakten des gegen Depressionen wirkenden Johanniskrauts und Digoxin. In ihrer einzelblinden, placebo-kontrollierten Parallelstudie fanden sie nach der kombinierten Einnahme von Digoxin und Extrakt eine signifikante Abnahme der Digoxinkonzentration im Plasma im Vergleich zur Placebo-Gruppe. Ruschitzka et al. (2000) beschreiben zwei Fälle von akuter Herz-Transplantat-Abstoßung nach der Einnahme von Johanniskraut. Beide Patienten wurden immunsuppressiv mit Ciclosporin, Azathioprin und Corticosteroiden behandelt. Nach Beginn der Einnahme fielen die Konzentrationen des Ciclosporins unter die therapeutische Grenze. In beiden Fällen konnte bioptisch eine akute Abstoßungsreaktion nachgewiesen werden. Nach Absetzen des Hypericum-Extraktes kehrten die Konzentrationen in den therapeutischen Bereich zurück. Es konnten keine anderen Ursachen für die Abstoßungsreaktion gefunden werden. Die zeitliche Relation zum Beginn der Johanniskrautbehandlung ließ auf einen Zusammenhang schließen. 
Miller gibt in ihrem Artikel von 1998 einen Überblick über bekannte und potenzielle Interaktionen von pflanzlichen Produkten. Echinacea wurde mit hepatotoxischen Effekten bei langer Einnahme in Verbindung gebracht. Es könne also vor allem mit anderen hepatotoxischen Präparaten (Amiodaron, Methotrexat, Ketoconacol) zu Interaktionen kommen. Mögliche Interaktionen sieht sie ebenfalls mit Corticosteroiden und Cyclosporinen in Form einer Aufhebung der immunsuppresiven Wirkung. Ginkgo, das vermehrt zur Behandlung der Demenz eingesetzt wird, könne nach Miller die Effektivität von Antikonvulsiva vermindern (Miller 1998).

\subsubsection{Missbrauch}

Ein Fünftel der 1,5 Millionen Medikamentenabhängigen in Deutschland soll, nach Glaeske, aus dem Bereich der Selbstmedikation stammen. Bei den Schmerzmitteln führen Acetaminophen, ASS und Ibuprofen die „TOP 10“ der US Abuse Warning Network Statistik an (Abbott und Fraser 1998).

OTC-Analgetika werden nicht allein gegen Schmerzen eingenommen sondern oft schon morgens, um die psychischen Belastungen und den Stress des Tages zu bewältigen (Abbott und Fraser 1998). Nach einer weitverbreiteten Meinung könnten NSARs nicht tödlich sein. Dies ergab eine Studie, in der 169 Highschool-Studenten in Florida per Fragebogen befragt wurden. 40,5\% unterschätzten die potenzielle Letalität von Acetaminophen. 17\% glaubten, Acetaminophen könne nicht tödlich sein (Myers et al. 1992).

\subsection{Selbstmedikation und Hausarzt-Patient-Beziehung}

Oben angesprochene Probleme betreffen nicht nur die Konsumenten rezeptfreier Arzneimittel, sondern in großem Maße auch den behandelnden Hausarzt. Aufgrund genannter Gefahren, ist es sehr wichtig, dass der Arzt von den OTC-Präparaten seines Patienten weiß. Medikation ohne ärztliche Verordnung heißt keineswegs, dass diese Art der Behandlung völlig am Hausarzt vorbei gehen darf. Um die Gefahren der Selbstmedikation zu minimieren ist es unbedingt nötig, Aufklärung zu betreiben (Kennedy 1996; Editorial Lancet 1994). Diese sollte vor allem vom Hausarzt angeboten werden. Für Blenkinsopp und Bradley (1996) ist "collaborative care" das essen- 
zielle Stichwort im Zusammenhang mit Selbstmedikation. Sie, wie auch andere Autoren (Editorial Lancet 1994), sehen Selbstmedikation als eine positive Entwicklung, wenn die Verantwortlichen im Gesundheitssystem (Ärzte, Apotheker, Schwestern und Pfleger) enger zusammenarbeiten. Auf diese Art und Weise könnten die Gefahren der Selbstmedikation minimiert werden.

Inwieweit Selbstmedikation Thema im Sprechzimmer des Hausarztes ist und ob Patienten dieses wünschen und gutheißen würden, soll Thema dieser Studie werden. Außerdem soll ein besonderes Augenmerk auf potenzielle Interaktionen zwischen verschriebenen und OTC-Medikamenten gerichtet werden. 


\section{Fragestellung und Hypothesen}

In dieser Studie sollte mit Hilfe einer Befragung von Hausärzten und Patienten untersucht werden, ob Selbstmedikation zu einem Thema in der Sprechstunde des Hausarztes geworden ist. Im Vordergrund stand dabei

(1) die Art der Selbstmedikation

(2) die Kommunikation mit dem Hausarzt über Selbstmedikation

(3) die Bewertung von Selbstmedikation durch Hausärzte

(4) die parallele Einnahme von verordneter Medikation und Selbstmedikation und daraus resultierenden möglichen Risiken.

Für die Durchführung der Untersuchung wurden folgende Hypothesen zugrunde gelegt:

1. Vor allem Frauen und Alleinlebende verwenden Selbstmedikation bevor sie den Hausarzt konsultieren (vgl. Figueiras et al. 2000).

2. (Kopf)schmerzen, Verdauungsprobleme und Erkältungskrankheiten behandeln Patienten - nach eigener Angabe - häufiger selbst als andere akute und chronische Krankheiten (vgl. BAH 1999).

3. Vor allem Patienten mit vielen verschriebenen Medikamenten verwenden Selbstmedikation.

4. Patienten, die selten den Hausarzt aufsuchen verwenden häufiger Selbstmedikation als solche, die regelmäßig zum Hausarzt gehen.

5. Mehr als die Hälfte aller Patienten hat noch nicht mit ihrem Hausarzt über Selbstmedikation gesprochen.

6. Vor allem Frauen, Ältere, Personen in Mehrpersonen-Haushalten und mit geringerer Schulbildung sprechen mit dem Hausarzt über Selbstmedikation. 
7. Über die Hälfte der Patienten wünschen das Gespräch mit dem Hausarzt über ihre Selbstmedikation (vgl. Bradley et al. 1998).

8. Gegenüber Hausärzten, die der Selbstmedikation negativ eingestellt sind, erwähnen weniger Patienten ihre Selbstmedikation.

9. Nach Patientenangaben geht das Gespräch über Selbstmedikation häufiger vom Patienten aus als vom Hausarzt.

10. Obwohl Hausärzte es für wichtig halten von der Selbstmedikation ihrer Patienten zu wissen, fragen - zumindest in der Wahrnehmung von Patienten - über die Hälfte der Hausärzte diese Medikamente nicht ab (vgl. Holden 1992).

11. Ärzte empfehlen Selbstmedikation häufiger bei Patienten zwischen 20 bis 35 Jahren und mit „Medikamenten-Erfahrung“. Dies spiegelt sich sowohl in den Aussagen der Ärzte, als auch der Patienten wider (vgl. Pradel et al. 1999). 


\section{Methoden}

Zum Thema Selbstmedikation sollten Hausärzte (Allgemeinärzte, Praktische Ärzte und hausärztlich tätige Internisten) und ihre Patienten persönlich befragt werden. Befragt wurden Erwachsene ab 18 Jahren. Die Daten wurden an jeweils einem Vorbzw. Nachmittag in den Praxen mittels persönlicher Befragung erhoben.

\subsection{Auswahl der Arztpraxen}

Als Studienort wurde die Grafschaft Bentheim in Niedersachsen gewählt. Alle niedergelassenen Allgemeinärzte und Praktischen Ärzte wurden mit Hilfe des Ärzteverzeichnisses angeschrieben bzw. angerufen und zur Teilnahme an der Studie eingeladen. Auf einem Antwortbogen, der an die Abteilung Allgemeinmedizin zurückgesandt werden sollte, konnten die Ärzte vermerken, ob sie an der Studie teilnehmen würden oder nicht.

Ärzte, die zwei Wochen nach Briefversand noch nicht geantwortet hatten, wurden noch einmal telefonisch zur Teilnahme eingeladen. Auf diese Weise konnte bereits bei diesem Telefonat ein Termin mit Ärzten, die einer Teilnahme zustimmten, festgelegt werden. Circa zwei Wochen vor dem jeweiligen Befragungstermin erfolgte ein Telefonat mit den Arzthelferinnen, in dem sie über den Ablauf der Studie informiert wurden und in dem geklärt wurde, ob ein separater Raum für die Patientenbefragung zur Verfügung stünde. Ebenfalls in diesem Gespräch wurde geklärt, wie lang die durchschnittliche Wartezeit der Patienten in dieser Praxis sei.

\subsection{Patienten-Fragebogen}

Ziel dieser Studie war es, ein möglichst komplexes Bild des Selbstmedikationsverhaltens von Hausarzt-Patienten - auch mit Bezug auf ihre verordnete Medikation - zu erhalten. Weil das Thema Selbstmedikation kein Tabuthema ist, wurden die Patientendaten mittels persönlicher Befragung erhoben. Um das Interview zu standardisieren, hatte der Fragebogen überwiegend "geschlossene" Fragen und Antwortvorgaben (Anhang 11.1, S. 85). Auch die Vorstellung der Studie bei den Patienten erfolgte mit einem standardisierten Text - mit situationsbedingten Varianten. 
Neben soziodemographischen Fragen, fokussierte der Patienten-Fragebogen auf folgende Bereiche:

- aktueller Konsultationsanlass

- Häufigkeit der Besuche beim Hausarzt

- chronische Erkrankungen

- Medikamenteneinnahme für aktuelle und chronische Leiden, Schmerzen, Erkältung, Verdauung und andere Beschwerden

- Gespräch mit dem Hausarzt über Selbstmedikation

- Gesundheitszustand.

\subsection{Arzt-Fragebogen}

Im Gegensatz zum Patienten-Fragebogen, wurde bei den Ärzten auf die "offene" Befragung mit Hilfe eines Leitfaden-Interviews zurückgegriffen (Anhang 11.2, S. 91). Bis auf wenige Ausnahmen konnten die Ärzte frei antworten. Neben praxisstatistischen Daten ging es um folgende Themen:

- Einstellung zur Selbstmedikation

- Wichtigkeit des Wissens um die Selbstmedikation der Patienten

- Häufigkeit und Art der Gespräche über Selbstmedikation mit Patienten

- ärztliche Empfehlungen zur Selbstmedikation.

\subsection{Pilotstudie}

Am Donnerstag, den 07.12.2000 fand eine Probeerhebung in einer Allgemeinarztpraxis im Raum Göttingen statt. Die Patientenbefragung erstreckte sich von 8:00 Uhr bis 11:45 Uhr. Im Anschluss folgte das Gespräch mit dem Hausarzt. Sämtliche Patienten (7 Frauen und 6 Männer), die um Teilnahme gebeten wurden, waren bereit, die Fragen zu beantworten.

Weil die meisten Patienten bereit waren, ggf. auch nach dem Arztbesuch auf die Befragung zu warten, konnten fast alle Patienten interviewt werden, die an diesem Morgen die Praxis aufsuchten. 
Der Ort des Gesprächs variierte, je nachdem welcher Raum gerade zur Verfügung stand. Oft konnte das (in diesem Fall leere) Wartezimmer bei geschlossener Tür zur Befragung genutzt werden; ansonsten diente der Bestrahlungsraum oder das Labor als Ort des Interviews.

\section{Allgemeine Probleme}

- Weil die Wartezeit oft weniger als 15 Minuten betrug, konnte der übliche Ablauf (Befragung während der Wartezeit) nicht ausgetestet werden.

- An diesem Morgen war „wenig Betrieb“ in der Praxis. Daher konnte nicht getestet werden, ob es auch möglich sein würde, dass vom Personal nur jeder 2. bzw. 3. Patient angesprochen und interviewt wird.

- Im Falle einer Überschneidung mit der Behandlung durch den Arzt, war es hier möglich, dass der Arzt auf den Interviewer gewartet hat. Dies würde in vielen Praxen nicht möglich sein.

Aufgrund der Probleme, die während der Pilotstudie auftraten, wurde das Studienprotokoll für den Ablauf und die Fragebogen präzisiert.

\subsection{Auswahl der Patienten und Ablauf der Befragung}

Am Erhebungstag wurden in der jeweiligen Praxis noch einmal die Arzthelferinnen über den Ablauf der Befragung informiert. Außerdem wurde bei Einverständnis des Arztes ein Informationszettel ausgehängt, in dem über die Befragung informiert wurde (Anhang 11.4, S. 98).

Die Patienten sollten während ihrer Wartezeit befragt werden, um eine höhere Compliance zu erreichen. Auf diese Art mussten sie keine zusätzliche Zeit in der Praxis verbringen. Um den Praxisablauf nicht zu stören, sollten nur Patienten befragt werden, die gerade die Praxis betreten hatten. Die Patienten wurden gleich nach der Anmeldung von den Arzthelferinnen über die Studie informiert. Bei Bereitschaft zur 
Teilnahme erhielten die Patienten ein Informationsblatt (informed consent), indem die Studie ausführlich erklärt wurde (Anhang 11.5, S. 100).

Das Gespräch sollte standardisiert beginnen:

1. Persönliche Vorstellung.

2. Erklärung der Studie.

3. Hinweise zur Anonymität und Datenschutz.

Das gesamte Gespräch mit dem Patienten sollte in einem separaten Raum geführt werden, um dem Patienten eine Privatsphäre zu bieten. War dies nicht möglich, sollte versucht werden, eine weitgehend abgeschirmte Situation zu gewährleisten. Wurde der Patient während der Befragung aufgerufen, sollte - bei Einverständnis des Patienten - nach der Konsultation das Interview fortgeführt werden. Dadurch ließ sich eine Störung des normalen Praxisablaufs vermeiden.

Am Ende der Sprechstunde war das Interview mit dem Arzt vorgesehen. Dieses Gespräch war auf ca. fünfzehn Minuten angelegt.

\subsection{Datenanalyse}

Die Medikamente wurden mit Hilfe des Anatomisch-Therapeutisch-Chemischen Klassifikationssystems der WHO (ATC-Code) verschlüsselt (Schwabe 1995). Die Konsultationsanlässe wurden mit der "International Classification of Primary Care" (ICPC) (WONCA International Classification Committee 2001) codiert. Auf diese Weise konnten Medikamente und Diagnosen in der internationalen Klassifikation in die Statistik mit eingehen.

Die Auswertung der Daten erfolgte mit dem Statistikprogramm SAS in der Version 8.02 für Windows. Es wurden zunächst univariate und bivariate Häufigkeitsauszählungen durchgeführt. Mögliche Einflüsse auf bestimmte Meinungen der befragten Patienten zur Selbstmedikation wurden in mehreren multiplen logistischen Regressionen überprüft (multivariat adjustiert). Als Effektgrößen wurden die Odds Ratios (ORs) mit 95\%-Konfidenzintervall (KI) berechnet (SAS Institute 1999). 


\subsection{Medikamenten-Interaktionen}

Um zu erfahren, ob es bei den befragten Patienten durch die Einnahme von Selbstmedikation zu potenziellen Interaktionen mit verschriebenen Medikamenten kommen könnte, wurde das Vorkommen bestimmter Medikamentenkombinationen anhand der Angaben der Patienten über verordnete Medikamente und den Kauf von OTCPräparaten untersucht. Vorbild war dabei die Studie von Sihvo et al. (2000). Die Betrachtung wurde auf folgende, möglicherweise häufige MedikamentenKombinationen beschränkt: (1) ASS und ACE-Hemmer sowie (2) Ibuprofen und Betablocker bzw. Diuretika. Weiterhin wurde überprüft, ob - im Fall des Auftretens einer o.g. Kombination - der Hausarzt darüber informiert war. Dies wurde angenommen wenn der Patient die Frage: "Haben Sie über dieses konkrete Medikament mit Ihrem Hausarzt gesprochen?“ bejahte.

\subsection{Datenschutz und Ethik}

Bei Bereitschaft zur Teilnahme an der Studie wurde den Patienten von den Arzthelferinnen ein Informationsbogen (Anhang 11.5, S. 100) überreicht. Vor der Befragung erläuterte die Doktorandin noch einmal, dass die Angaben während des Interviews völlig anonym behandelt würden und auch der Arzt oder das Praxispersonal keine Einsicht erhielten. Außerdem wurden eventuelle Fragen des Patienten zum Datenschutz beantwortet. Es wurden keine Namen, Geburtsorte oder -daten der Patienten und Ärzte auf dem Fragebogen dokumentiert. Die Eingabe in SAS erfolgte ebenfalls anonym. Praxen und Patienten wurden als Kennziffern eingegeben.

Die Studie wurde durch die Ethik-Kommission der Universität Göttingen genehmigt. 


\section{Ergebnisse}

\subsection{Patienten}

Von 346 Patienten, die zur Teilnahme eingeladen wurden, nahmen 280 Patienten in 20 Praxen am Interview teil, durchschnittlich 11 Patienten pro Praxis (Min.: 2, Max.: 18). 66 Patienten lehnten die Teilnahme ab (Teilnehmerquote: 80,9\%). Der häufigste Ablehnungsgrund war Zeitmangel $(n=23)$. In den meisten Fällen nannten die Patienten keinen Grund.

17 Befragte wurden aus den Berechnungen ausgeschlossen, da sie - abweichend von den Einschlusskriterien - entweder noch nicht das 18. Lebensjahr vollendet hatten oder der konsultierte Arzt nicht der Hausarzt war. Von den verbliebenen $263 \mathrm{~Pa}$ tienten waren $162(61,6 \%)$ Frauen. Das mittlere Alter der befragten Patienten lag bei 50,5 Jahren (Min.: 18, Max.: 87, Median: 50). Die Männer waren mit 54,3 Jahren im Mittel etwas älter als die Frauen $(48,1)$. In Tabelle 4 sind weitere Merkmale der Patienten aufgeführt.

Durchschnittlich kamen die interviewten Patienten - nach eigenen Angaben - ca. zehnmal im Jahr zu ihrem Hausarzt (Min.: 0, Max.: 350 ${ }^{1}$ ). Bei einem Minimum von 0 und einem Maximum von 30 Jahren waren die Befragten im Mittel seit 11,7 Jahren bei ihrem Hausarzt in Behandlung.

Der häufigste Konsultationsgrund waren Rückenbeschwerden (35 Patienten). Ein neues Rezept und Blutabnahmen waren ebenfalls häufig genannte Gründe (Tabelle 5). Drei Viertel (196 / 263) der befragten Patienten litten unter mindestens einer chronischen Erkrankung. Von diesen Patienten ließen 96\% ihre chronische Krankheit durch den Hausarzt, 4\% ausschließlich durch Fachärzte behandeln. Fast alle (169) dieser Patienten nahmen mindestens ein Medikament gegen ihre chronischen Beschwerden ein.

\footnotetext{
${ }^{1}$ Nur ein Patient gab an, den Hausarzt fast jeden Tag zur Kontrolle des Blutdruckes aufzusuchen.
} 
Tabelle 4: Patientenmerkmale*

\begin{tabular}{|c|c|c|}
\hline Geschlecht (263) & $\mathbf{n}$ & $\%$ \\
\hline Männlich & 101 & 38,4 \\
\hline Weiblich & 162 & 61,6 \\
\hline \multicolumn{3}{|l|}{ Alter (263) } \\
\hline $18-25$ & 24 & 9,1 \\
\hline $26-35$ & 36 & 13,6 \\
\hline $36-45$ & 53 & 20,1 \\
\hline $46-55$ & 39 & 14,8 \\
\hline $56-56$ & 46 & 17,4 \\
\hline Über 56 & 65 & 24,7 \\
\hline \multicolumn{3}{|l|}{ Schulabschluss (260) } \\
\hline Keinen & 9 & 3,4 \\
\hline Haupt- / Volksschule & 148 & 56,9 \\
\hline Realschule & 73 & 28,0 \\
\hline Fachhochschule & 11 & 4,2 \\
\hline Abitur & 19 & 7,3 \\
\hline \multicolumn{3}{|l|}{ Haushaltsgröße (263) } \\
\hline Allein im Haushalt & 39 & 14,8 \\
\hline Mehrpersonen-Haushalt & 224 & 85,1 \\
\hline \multicolumn{3}{|l|}{ Zuzahlung (261) } \\
\hline Nicht befreit & 171 & 65,5 \\
\hline davon chronisch krank & 116 & 67,8 \\
\hline Befreit & 84 & 32,1 \\
\hline davon chronisch krank & 72 & 85,7 \\
\hline Privat versichert & 6 & 2,3 \\
\hline davon chronisch krank & 6 & 100,0 \\
\hline
\end{tabular}

* In Klammern jeweils die Zahl (n) der insgesamt die Frage beantwortenden Patienten (^ $100 \%)$.

Der selbstbeurteilte Gesundheitszustand auf einer Skala von "ausgezeichnet" (= 1) bis "schlecht“ (= 5) war im Mittel "gut" $(3,2)$. Frauen beurteilten ihren Gesundheitszustand etwas schlechter als Männer (Abbildung 1). 
Tabelle 5: Häufigste Gründe für die Arztkonsultation*

\begin{tabular}{ll}
\hline Gründe für die Arztkonsultation & $\mathbf{n}$ \\
\hline Rückenschmerzen & 35 \\
Rezept & 28 \\
Blutabnahme & 22 \\
Andere nicht klassifizierte Anlässe & 22 \\
Akuter Infekt der oberen Luftwege & 20 \\
Untersuchungsergebnisse & 18 \\
Bluthochdruck & 16
\end{tabular}

* Die hier aufgeführten Gründe repräsentieren $61 \%$ aller genannten Konsultationsgründe.

\subsection{Einnahmeverhalten der Patienten}

Insgesamt 245 (93,2\%) der befragten Patienten gaben an, mindestens ein Medikament in den letzten 4 Wochen eingenommen zu haben. Bei 80,6\% der Patienten handelte es sich um vom Arzt verschriebene Medikamente.

104 Patienten (39,5\%) hatten bereits vor dem Arztbesuch ein Medikament gegen ihre aktuellen Beschwerden eingenommen, vor allem bei Beschwerden im Muskelund Skelettsystem (14\%), Respirationssystem (9\%) und ZNS (8\%). Medikamente gegen chronische Beschwerden nahmen 63,5\% der Befragten ein. Die Spanne reichte von einem (13,7\%) bis zu 7 (3,4\%) Medikamenten (Tabelle 6).

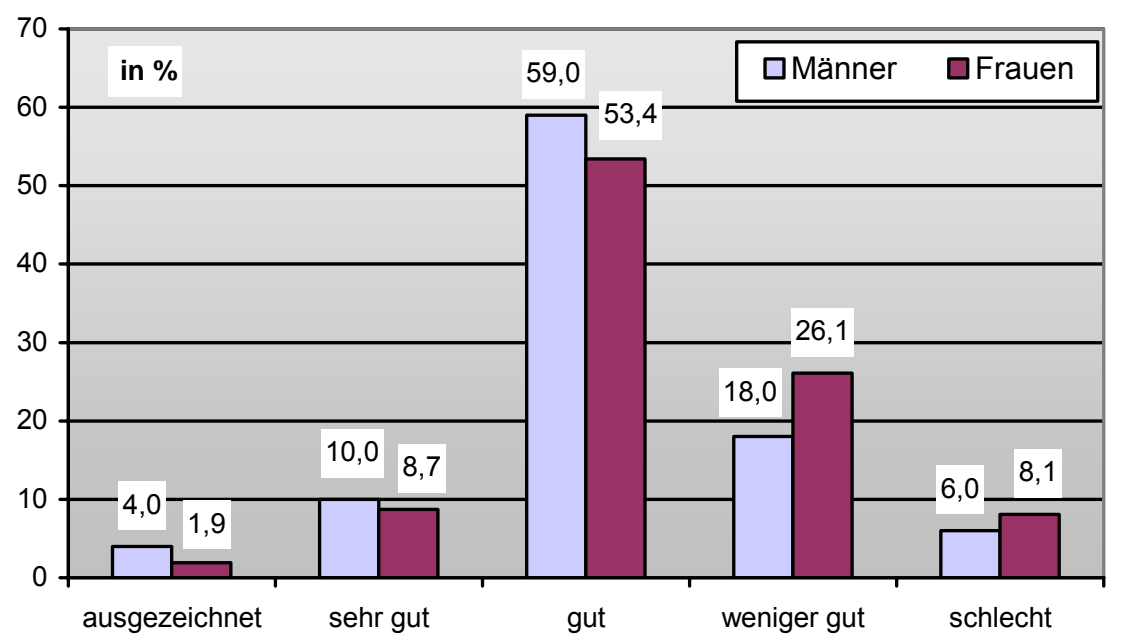

Abbildung 1: Selbsteingeschätzter Gesundheitszustand 
Tabelle 6: Aktuelle Einnahme von Medikamenten gegen chronische Beschwerden

\begin{tabular}{lcccccccc}
\hline \multirow{2}{*}{ Patienten } & $\mathbf{0}$ & $\mathbf{1}$ & $\mathbf{2}$ & $\mathbf{3}$ & $\mathbf{4}$ & $\mathbf{5}$ & $\mathbf{6}$ & $\mathbf{7}$ \\
\cline { 2 - 9 } & 96 & 36 & 49 & 29 & 20 & 13 & 11 & 9 \\
$\mathbf{N}$ & 36,5 & 13,7 & 18,6 & 11,0 & 7,6 & 4,9 & 4,2 & 3,4 \\
\hline
\end{tabular}

Bei diesen Medikamenten handelte es sich vor allem um Präparate für das HerzKreislauf-System (45,3\%), das Verdauungssystem $(19,8 \%)$ sowie systemische Hormonpräparate $(17,1 \%)$.

Medikamente gegen Schmerzen nahmen 214 Befragte $(81,4 \%)$ ein, fast $60 \%$ hatten dies auch in den letzten 4 Wochen vor der Befragung getan. Am häufigsten angewandt wurden nichtsteroidale Antiphlogistika (vor allem Salicylderivate, Pyrazolonderivate), Anilinderivate (vor allem Paracetamol) und topische Schmerzmittel. Schmerzmittelkombinationen nahmen 8,4\% der Befragten ein. Homöopathische Mittel wurden nicht genannt (Tabelle 7).

Tabelle 7: Verwendete Schmerz- und Erkältungsmittel (Wirkstoffe)

\begin{tabular}{lcc}
\hline Schmerzmittel & $\mathbf{n}$ & $\%$ \\
\hline Salicylderivate & 109 & 41,4 \\
Anilinderivate & 64 & 24,3 \\
Topische Schmerzmittel & 63 & 24,0 \\
NSAR & 32 & 12,2 \\
Kombination & 22 & 8,4 \\
Pyrazolonderivate & 11 & 4,2 \\
Opioide & 8 & 3,0 \\
Migränemittel & 4 & 1,5 \\
\hline Erkältungsmittel & $\mathbf{n}$ & $\%$ \\
\hline Erkältungspräparate* & 140 & 52,2 \\
Kombinationen & 98 & 37,3 \\
Expektoranzien / Mukolytika & 76 & 28,9 \\
Stomatologische Präparate & 56 & 21,3 \\
Rhinologika & 55 & 20,9 \\
Homöopathische / Pflanzliche Mittel & 35 & 13,3 \\
Analgetika & 20 & 7,6 \\
Antitussiva & 6 & 2,3 \\
\hline
\end{tabular}

\footnotetext{
* Im ATC-Code als „Husten- und Erkältungspräparate“ klassifiziert. Hierzu zählen auch pflanzliche und homöopathische Mittel.
} 
Der Anteil der Befragten, der Medikamente für Erkältungskrankheiten einnahm, war mit 68\% ( $n=179)$ ebenfalls hoch; in den letzten 4 Wochen hatten 37,6\% ein Medikament eingenommen. Tabelle 7 zeigt die häufigsten verwendeten Wirkstoffe. Wirkstoffkombinationen und auch homöopathische Mittel spielten hier eine größere Rolle. Verdauungsmedikamente nahmen - mit deutlichem Abstand - den dritten Platz ein. $13,3 \%$ der Befragten $(n=35)$ nahmen ein Medikament ein: vor allem Laxanzien (16 Patienten) sowie Antacida und Ulkustherapeutika (9 Patienten). In der Gruppe „andere Medikamente“ wurden Medikamente für das ZNS $(11,4 \%)$ und Präparate für die Haut $(6,8 \%)$ am häufigsten angegeben. Tabelle 8 fasst die Ergebnisse zusammen.

Tabelle 8: Einnahmeverhalten bei Schmerzen, Erkältung, Verdauungsproblemen und anderen Beschwerden

\begin{tabular}{lcc}
\hline & $\mathbf{n}$ & \% \\
\hline Medikament für Schmerzen & 214 & 81,4 \\
- Einnahme in letzten 4 Wochen vor der Befragung & 154 & 58,6 \\
& & \\
Medikament für Erkältungskrankheiten & 179 & 68,1 \\
- Einnahme in letzten 4 Wochen vor der Befragung & 99 & 37,6 \\
Medikament für Verdauungsprobleme & 35 & 13,3 \\
- Einnahme in letzten 4 Wochen vor der Befragung & 26 & 9,9 \\
& & 38,4 \\
Medikament für andere Beschwerden & 101 & 30,4 \\
\hline
\end{tabular}

\subsubsection{Verschriebene Medikamente}

Vor allem Patienten mit geringerem Schulabschluss (Abbildung 2), schlechter eingeschätztem Gesundheitszustand und von der Zuzahlung Befreite erhielten - nach eigenen Angaben - Medikamente auf Rezept verordnet. Auch mit dem Alter nahm die Einnahme von verschriebenen Medikamenten zu (Abbildung 3). Unterschiede zwischen Männern und Frauen sowie im Wohnstatus waren unerheblich (Tabelle 9). 


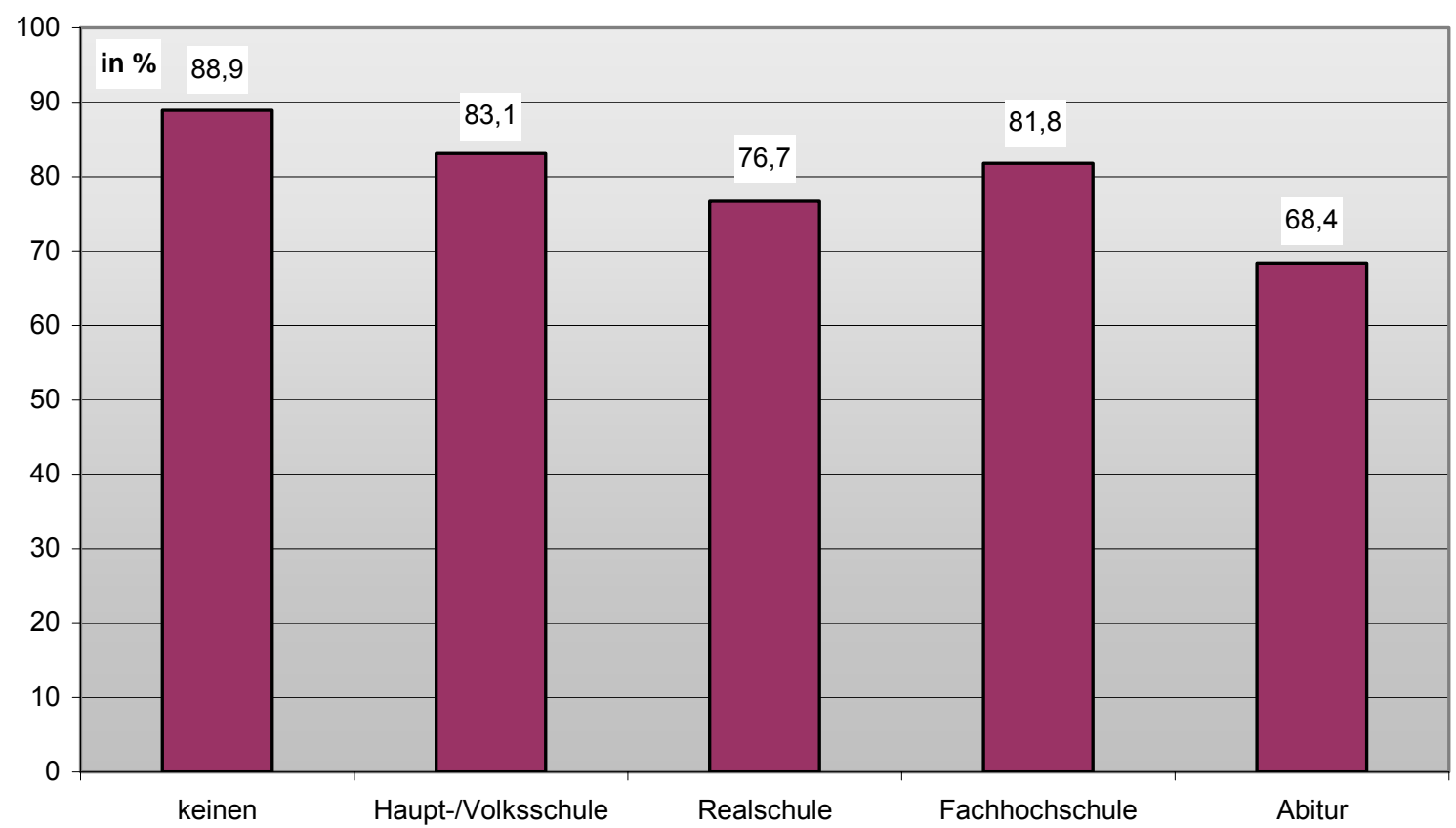

Abbildung 2: Verordnung von Medikamenten und Schulabschluss

\subsubsection{Selbstmedikation}

87,8\% (231 / 263) der Befragten nahmen Mittel ein, die sie ohne Rezept gekauft hatten. Viele von ihnen (189 / 231) hatten ein Medikament ohne Rezept in den 4 Wochen vor der Befragung eingenommen, vor allem bei Schmerzen und Erkältung. Mittel zur Selbstmedikation nutzten vergleichsweise häufiger Frauen und Alleinlebende (Abbildung 4). Patienten über 45 Jahre, chronisch Kranke und Privat-Versicherte nahmen seltener Medikamente ohne Rezept. Ebenfalls ist der Anteil Patienten, der Selbstmedikation betrieb, ohne Schulabschluss und mit Abitur kleiner als der bei Absolventen anderer Schulformen. Der selbsteingeschätzte Gesundheitszustand und die Einnahme von Dauermedikamenten schienen keinen bzw. keinen linearen Einfluss auf die Selbstmedikation gehabt zu haben (Tabelle 10).

Aufgeschlüsselt nach Indikationen (Verdauung, Schmerzen, Erkältung), nahmen etwas mehr ältere Patienten (über 55 Jahre) OTC-Verdauungsmittel (12,6\%; Durchschnitt aller Befragten, die diese Mittel eingenommen hatten: 8,8\%). 
Tabelle 9: Einnahme von verordneten Medikamenten*

\begin{tabular}{lc}
\hline Geschlecht (263) & Einnahme von verschriebenen Medikamenten \\
\hline Männer & $81,2 \%$ \\
Frauen & $80,3 \%$ \\
\hline Gesundheitszustand (261) & \\
\hline Ausgezeichnet & $57,1 \%$ \\
Sehr gut & $54,2 \%$ \\
Gut & $79,5 \%$ \\
Weniger gut & $90,8 \%$ \\
Schlecht & $100,0 \%$ \\
\hline Wohnstatus (263) & \\
\hline Mehrpersonen-Haushalt & $80,8 \%$ \\
Allein lebend & $79,5 \%$ \\
\hline Zuzahlung (261) & \\
\hline Nicht befreit & $73,7 \%$ \\
Befreit & $94,1 \%$ \\
Privat versichert & $83,3 \%$ \\
\hline
\end{tabular}

* In Klammern jeweils die Zahl ( $\mathrm{n})$ der insgesamt die Frage beantwortenden Patienten ( $\hat{=} \quad 100 \%)$.

Vergleichsweise viele Absolventen einer Fachhochschule nutzten ebenfalls OTCVerdauungsmittel (27,3\%; Durchschnitt aller Befragten: 8,9\%). Weiterhin gaben Patienten mit weniger gutem oder schlechtem Gesundheitszustand (Selbsteinschätzung) sowie chronisch Kranke zu einem größeren Anteil an, Medikamente gegen Verdauungsstörungen einzunehmen.

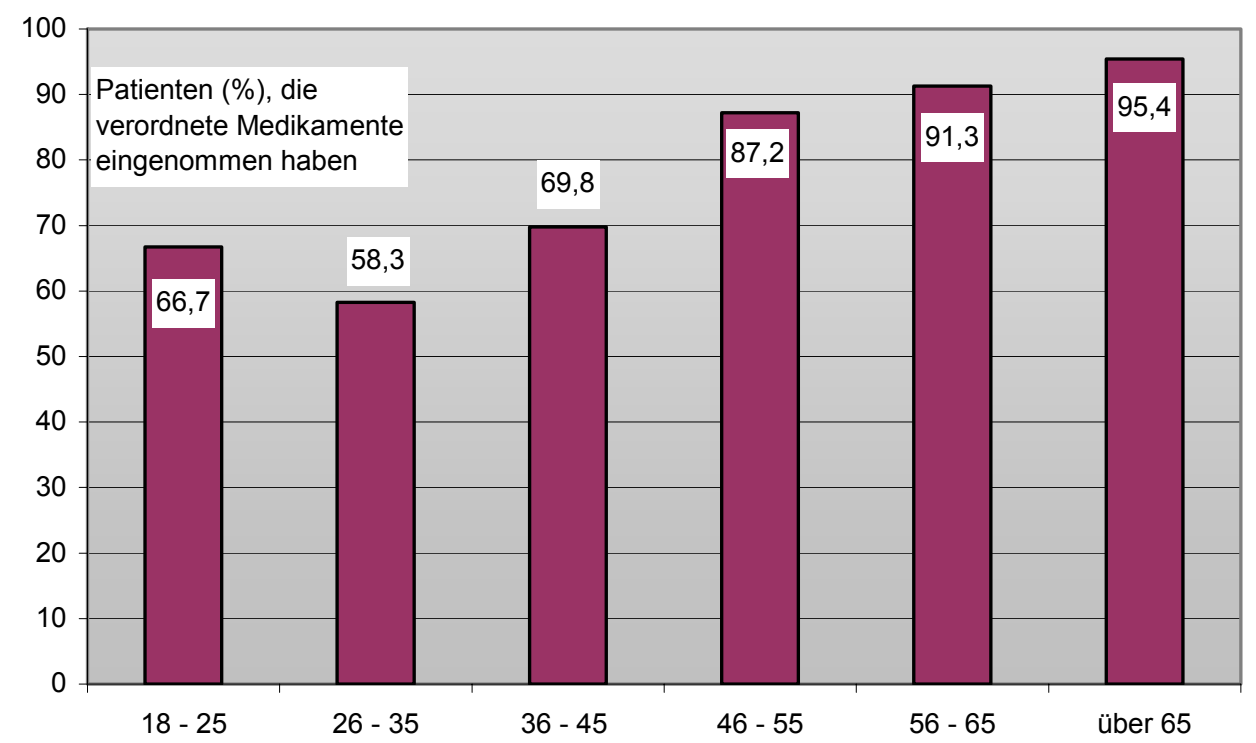

Abbildung 3: Verschriebene Medikamente und Alter 


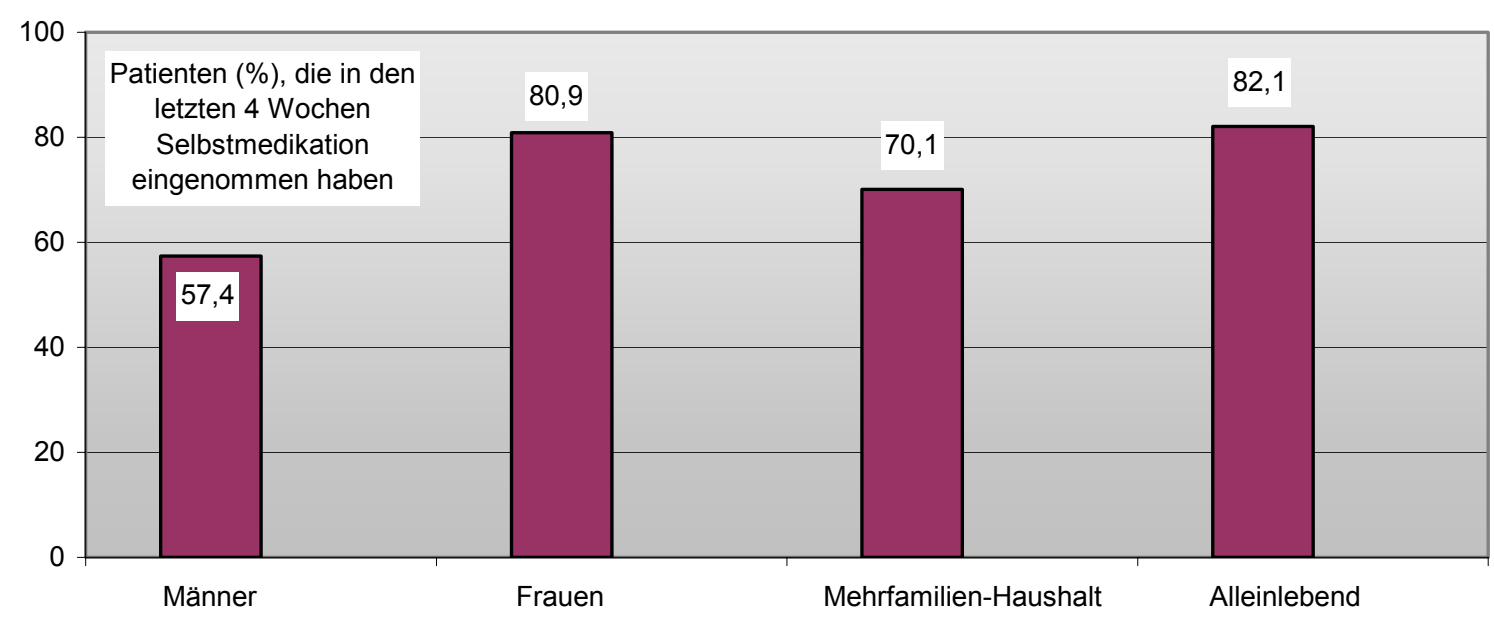

Abbildung 4: Selbstmedikation

Tabelle 10: Einnahme von Selbstmedikation in den letzten 4 Wochen*

\begin{tabular}{lc}
\hline Schulabschluss (260) & Einnahme von Selbstmedikation \\
\hline Keinen & $66,7 \%$ \\
Haupt- / Volksschule & $70,3 \%$ \\
Realschule & $75,3 \%$ \\
Fachhochschule & $72,7 \%$ \\
Abitur & $68,4 \%$ \\
\hline Gesundheitszustand (261) & \\
\hline Ausgezeichnet & $85,7 \%$ \\
Sehr gut & $58,3 \%$ \\
Gut & $69,0 \%$ \\
Weniger gut & $78,5 \%$ \\
Schlecht & $84,2 \%$ \\
\hline Einnahme Medikamente gegen chronische & \\
Beschwerden (263) & $70,0 \%$ \\
\hline Keine Einnahme & $73,0 \%$ \\
Einnahme eines oder mehrerer Medikamente & \\
\hline Zuzahlung (261) & $71,9 \%$ \\
\hline Nicht befreit & $71,4 \%$ \\
Befreit & $66,7 \%$ \\
Privat versichert & \\
\hline Chronische Krankheit (263) & $77,6 \%$ \\
\hline Nicht chronisch krank & $70,0 \%$ \\
Chronisch krank &
\end{tabular}

* In Klammern jeweils die Zahl (n) der insgesamt die Frage beantwortenden Patienten ( $\hat{=} 100 \%)$. 
OTC-Präparate gegen Schmerzen wurden vergleichsweise selten von Absolventen einer Fachhochschule genommen (27,3\%; Durchschnitt aller Befragten: 47,3\%). Auch chronisch Kranke gaben seltener den Gebrauch von OTC-Schmerzmitteln an. Patienten, die ihren Gesundheitszustand als ausgezeichnet einschätzten, berichteten mit $85,7 \%$ von einem überdurchschnittlich hohen OTC-Schmerzmittel-Konsum (Durchschnitt aller Befragten: 46,2\%). Auch Patienten unter 35 Jahren und Frauen nahmen in den letzten 4 Wochen vor der Befragung - nach eigenen Aussagen - besonders häufig selbsterworbene Schmerzmittel.

OTC-Erkältungsmittel wurden vor allem von Patienten unter 45 Jahren $(41,6 \%$; Durchschnitt aller Befragten: 34,2\%) und Alleinlebenden (41,0\%; Durchschnitt aller Befragten: $34,2 \%$ ) eingenommen.

\subsubsection{Zusammenhang zwischen der Anzahl an Dauermedikamenten und der Bereitschaft zur Einnahme von OTC-Präparaten}

Gibt es einen Zusammenhang zwischen der Anzahl an Dauermedikamenten und der Bereitschaft zur Selbstmedikation? Die folgende Auswertung bezieht sich auf die Selbstmedikation bei Schmerz, Erkältung und Verdauung in den letzten 4 Wochen vor der Befragung im Vergleich zu dauerhaft verordneten Medikamenten. Weiterhin wurde die Zahl aller im Monat vor der Befragung gekauften Selbstmedikationspräparate mit der Zahl verordneter Medikamente für chronische Krankheiten verglichen (Tabelle 11): Der Anteil Patienten, der OTC-Medikamente gegen Schmerzen einnahm, war geringfügig höher unter den Patienten, die mehr als 5 Medikamente (5 - 7 Medikamente) gegen chronische Beschwerden einnahmen. Mittel für die Verdauung dagegen wurden deutlich öfter von Patienten gekauft, die Dauermedikamente einnahmen, insbesondere von Patienten, die mehrere Dauermedikamente verordnet erhielten. Selbstmedikationspräparate gegen Erkältungskrankheiten verwendeten häufiger Patienten, die wenige oder keine Dauermedikamente einnahmen. Der Anteil mit dem stärksten Gebrauch an Selbstmedikation insgesamt fand sich in der Gruppe mit 5 bis 7 verordneten Medikamenten (83,3\%). Weder für alle Präparate der Selbstmedikation noch für einzelne Präparategruppen - mit Ausnahme der Verdauungsmittel - fand sich ein eindeutiger Zusammenhang zwischen der Einnahme von Dauermedikamenten und Selbstmedikation. Lediglich in der Gruppe der Patienten, 
die Verdauungsmittel selber erworben, ist ein stetiger Anstieg mit Zunahme der Dauermedikamente zu erkennen.

Tabelle 11: Selbstgekaufte Schmerz-, Verdauungs- und Erkältungsmittel und Anzahl "chronischer" Medikamente*

\begin{tabular}{lcccc}
\hline & \multicolumn{4}{c}{ Anzahl chronischer Medikamente } \\
\cline { 2 - 5 } Selbstmedikation & $\mathbf{0}$ & $\mathbf{1 - 2}$ & $\mathbf{3 - 4}$ & $\mathbf{5}$ und $\mathbf{~ m e h r}$ \\
$\mathbf{\%}$ & $\mathbf{\%}$ & 45,2 \\
\hline bei Schmerz & 48,0 & 45,2 & 45,7 & 54,2 \\
bei Erkältung & 35,0 & 35,5 & 23,9 & 45,8 \\
bei Verdauung & 5,0 & 9,7 & 10,9 & 16,7 \\
gesamt & 70,0 & 75,3 & 63,0 & 83,3 \\
\hline
\end{tabular}

* in den letzten 4 Wochen verwendet.

\subsubsection{Einnahme von Selbstmedikation und Einnahme anderer Medikamente}

Um heraus zu finden, ob Patienten mit bestimmten verordneten Medikamenten auch eher Präparate zur Selbstmedikation einnehmen, wurden - aus Gründen der Aussagekraft - nur Wirkgruppen betrachtet, die von über $3 \%(n \geq 9)$ der Patienten eingenommen wurden (Tabelle 12).

Alle Patienten, die Thrombozytenaggregationshemmer und / oder Anxiolytika einnahmen, betrieben auch Selbstmedikation. Auch Patienten mit stimmungsaufhellenden und schlaffördernden Mitteln (Sedativa, Antidepressiva) nahmen überdurchschnittlich häufig Präparate zur Selbstmedikation. Dies galt ebenfalls für Befragte denen - nach eigenen Angaben - Opioide, Analgetika ${ }^{2}$, topische Antirheumatika, NSAR und Medikamente gegen arteriellen Hypertonus (ACE-Hemmer, Betablocker, Kombinationen) verordnet wurden.

\footnotetext{
2 ohne NSAR, Opioide, Migränetherapeutika
} 


\subsubsection{Einnahme von Selbstmedikation, das Krankheitsbild und die Häufigkeit der Arztbesuche}

Besonders folgende Gruppen von Patienten hatten in den letzten 4 Wochen Selbstmedikation betrieben: Patienten mit Beschwerden im Bewegungsapparat (z.B. Schmerzen im Rücken oder Nacken, Gelenken etc.) (82,8\%), mit Erkrankungen der Atemwege (80\%) und mit Kreislaufbeschwerden (z.B. KHK, Herzrhythmusstörungen, Hypertonie) $(77,8 \%)$.

Tabelle 12: Einnahme bestimmter Medikamente und Selbstmedikation

\begin{tabular}{|c|c|c|}
\hline $\begin{array}{l}\text { Wirkungsbereich der } \\
\text { eingenommenen Medikamente }\end{array}$ & $\begin{array}{l}\text { Anteil am Gesamtkollektiv } \\
\qquad(n=263)\end{array}$ & $\begin{array}{c}\text { Patienten, die } \\
\text { Selbstmedikation betrieben }\end{array}$ \\
\hline & $\%$ & $\%$ \\
\hline Thrombozytenaggregationshemmer & 5,8 & 100,0 \\
\hline Anxiolytika & 4,2 & 100,0 \\
\hline Mineralstoffe* & 11,1 & 95,5 \\
\hline Laxanzien & 8,5 & 94,1 \\
\hline Opioide & 6,9 & 92,9 \\
\hline Galle-Therapeutika & 13,2 & 92,6 \\
\hline ACE-Hemmer & 5,3 & 90,9 \\
\hline Betablocker & 4,8 & 90,0 \\
\hline Hypnotika und Sedativa & 13,2 & 89,3 \\
\hline Stomatologika* & 27,5 & 88,1 \\
\hline Topische Antirheumatika & 33,9 & 86,5 \\
\hline Topische Rhinologika* & 25,9 & 86,0 \\
\hline Husten- und Erkältungsmittel ${ }^{*}$ & 65,6 & 85,5 \\
\hline Urologika & 5,3 & 83,3 \\
\hline NSAR & 24,9 & 82,5 \\
\hline Vitamine* & 12,2 & 82,1 \\
\hline Obstruktive Atemwegserkrankung & 9,5 & 81,8 \\
\hline Analgetika** & 75,1 & 81,1 \\
\hline Gastrointestinale Störungen & 4,2 & 80,0 \\
\hline Corticoide & 3,7 & 77,8 \\
\hline Antidepressiva & 5,3 & 76,9 \\
\hline $\begin{array}{l}\text { Kombination Betablocker / } \\
\text { Thiazidiuretikum }\end{array}$ & 4,2 & 72,7 \\
\hline
\end{tabular}

* Hoher Anteil an OTC-Päparaten.

** ohne NSAR, Opioide, Migränetherapeutika. 
Alle Patienten, die wegen neurologischer und psychischer Probleme den Hausarzt aufsuchten, gaben den Gebrauch von Selbstmedikation an (diese Gruppen sind allerdings mit insgesamt 6 bzw. 5 Patienten sehr klein und damit weniger aussagekräftig).

Bei den chronischen Erkrankungen sieht die Verteilung etwas anders aus. Der höchste Anteil an OTC-Nutzern fand sich in der Gruppe der Patienten mit neurologischen Problemen, wie Kopfschmerzen, Trigeminusneuralgie und multipler Sklerose (100\%). Chronische Verdauungsstörungen und Probleme mit den Atemwegen liegen mit $75 \%$ an zweiter Stelle. Patienten mit endokrinen und Stoffwechselproblemen (z.B. Schilddrüsenerkrankungen, Diabetes mellitus, Gewichtsverlust) sind mit 72,9\% an der dritten Stelle und entsprechen nahezu dem Durchschnittsgebrauch an Selbstmedikation.

Patienten, die sehr häufig ihren Hausarzt aufsuchten, kauften geringfügig seltener Präparate zur Selbstmedikation als der Durchschnitt (Abbildung 5).

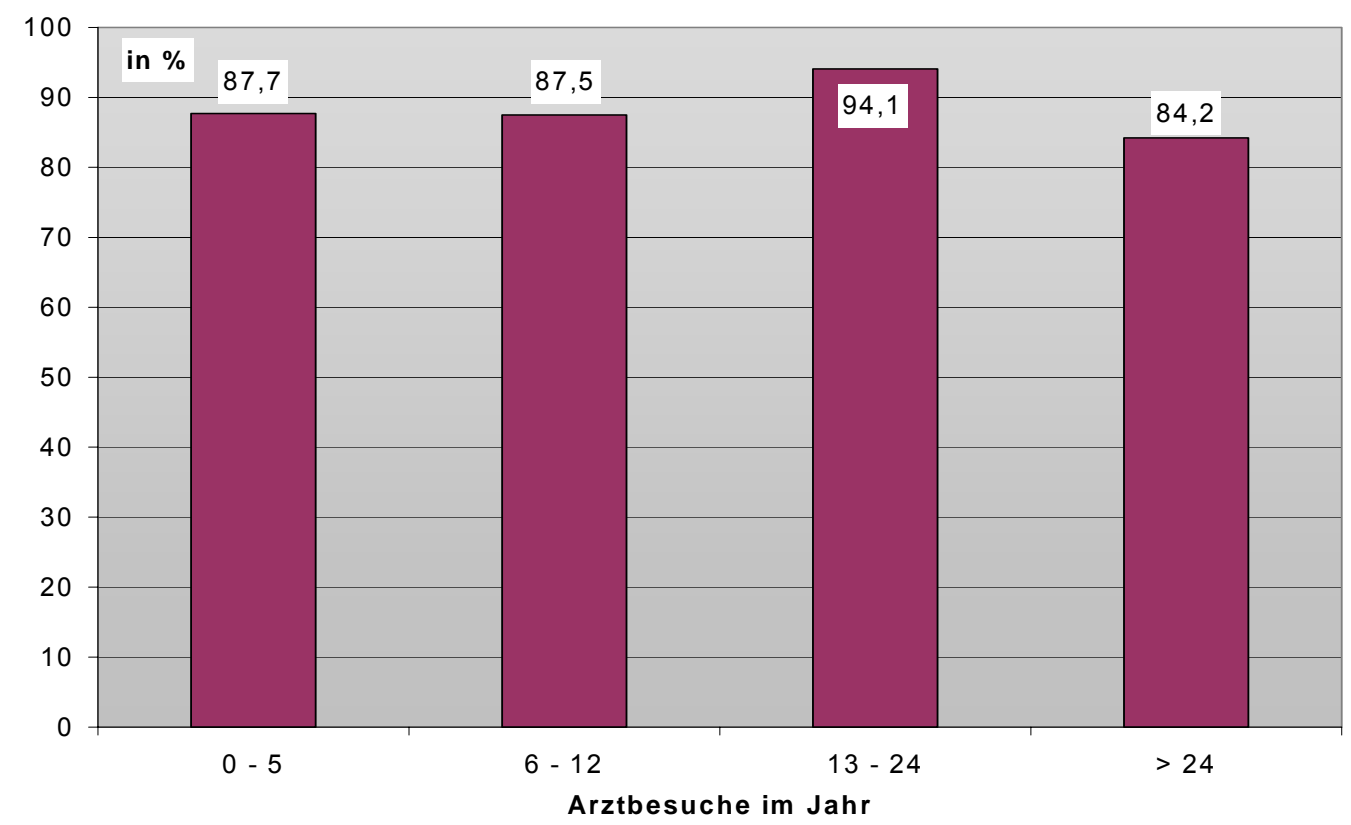

Abbildung 5: Selbstmedikation und die Häufigkeit der Arztbesuche 


\subsection{Kommunikation mit dem Hausarzt über Selbstmedikation}

Die Frage „Haben Sie sich generell schon einmal mit Ihrem Hausarzt über Medikamente, die Sie ohne Rezept gekauft haben, unterhalten?“ bejahte ein Drittel der Patienten - ohne Unterschied zwischen Männern (31 / 93) und Frauen (53 / 159). Nach Aussagen der Befragten ging in 60\% der Fälle das Gespräch von innen, in 40\% vom Arzt aus. Von den zwei Dritteln, die noch nie mit ihrem Hausarzt über Selbstmedikation gesprochen hatten, erinnerten sich 15 Befragte (8,9\%) zumindest einmal an eine Situation, in der sie dies gerne getan hätten.

Am häufigsten sprachen Patienten der Altersgruppe zwischen 36 und 45 Jahren über Selbstmedikation mit dem Hausarzt. Eine lineare Altersabhängigkeit gab es nicht (Abbildung 6). Weniger Alleinlebende als Patienten in Mehrpersonen-Haushalten hatten mit ihrem Hausarzt über Selbstmedikation gesprochen (25,6\% vs. $34,7 \%)$.

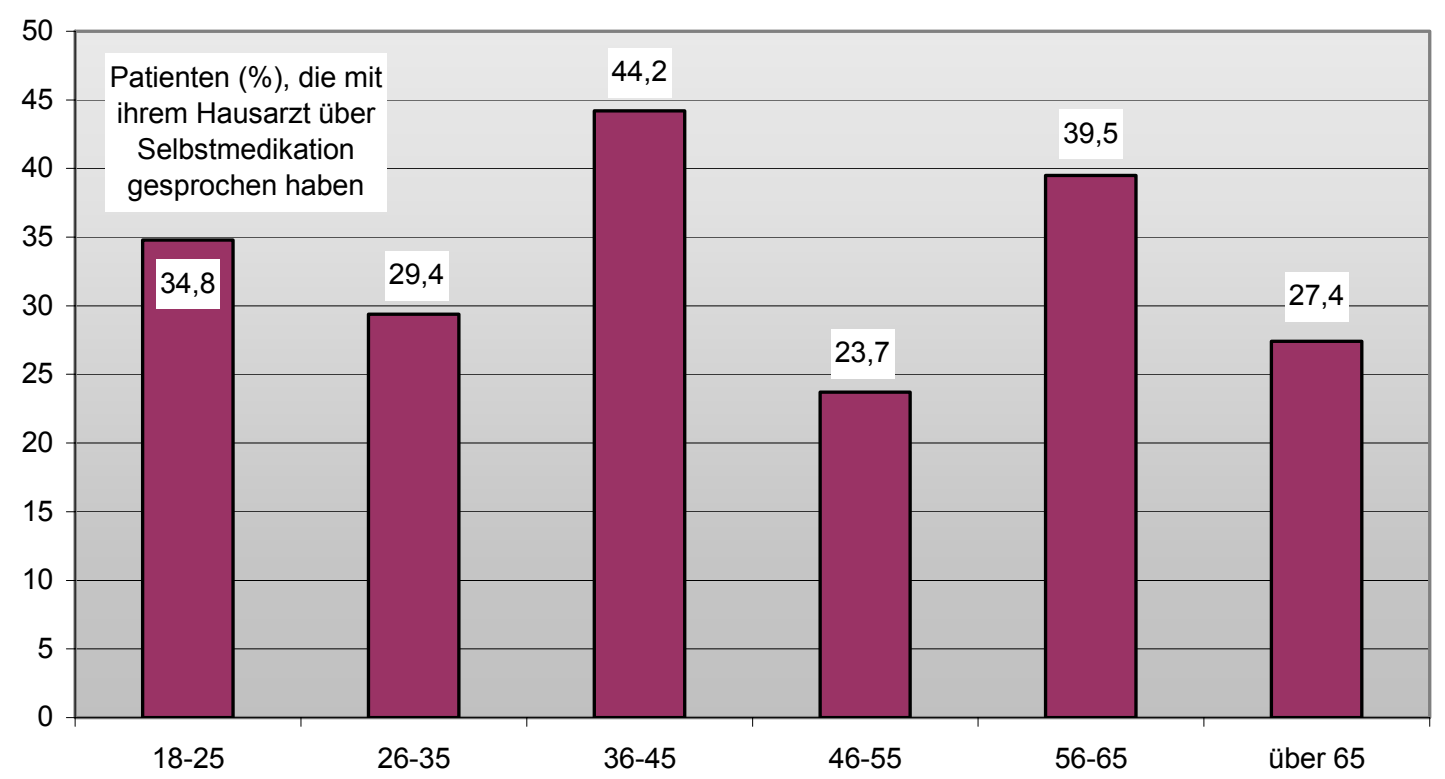

Abbildung 6: Unterhaltung mit dem Hausarzt über Selbstmedikation und Alter

Keine Unterschiede gab es in dieser Hinsicht zwischen Patienten mit Zuzahlung $(32,9 \%)$ bzw. Befreiung (33,3\%). Ein geringfügig höherer Anteil privat versicherter Patienten hatte schon einmal mit ihrem Hausarzt über Selbstmedikation gesprochen (40\%). Dies galt auch für Patienten mit einem höheren Bildungsstand (Abbildung 7), wobei beide Faktoren in wechselseitiger Abhängigkeit stehen. 


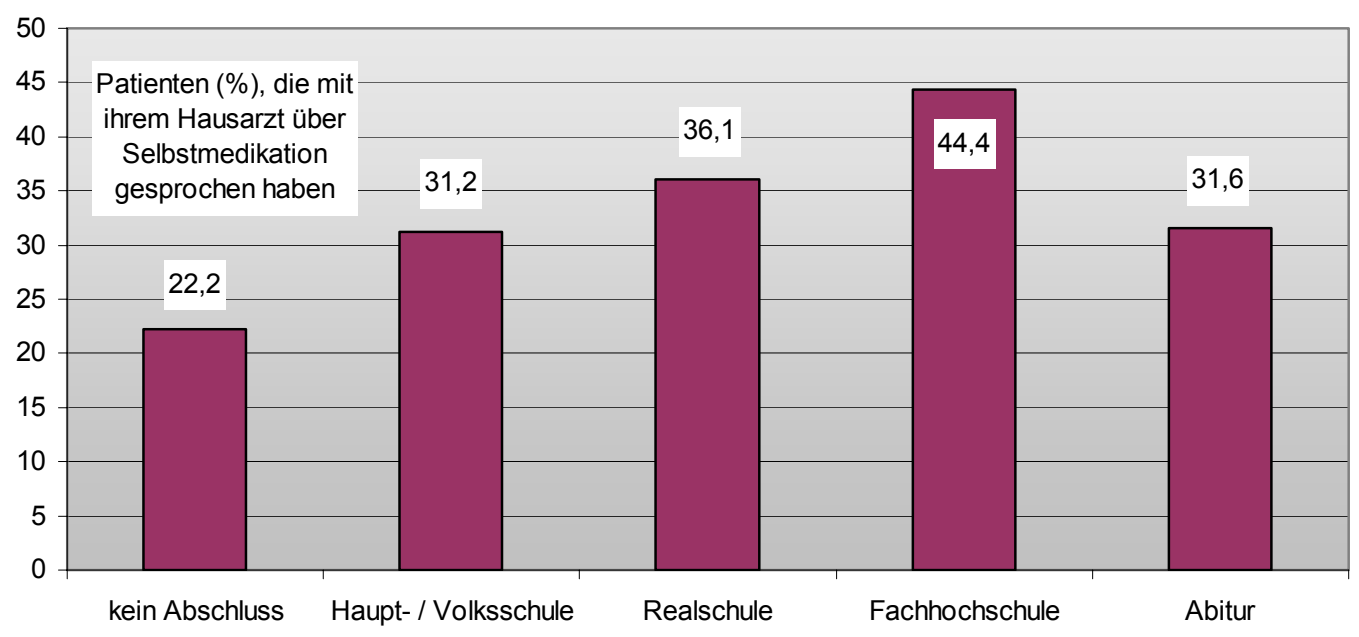

Abbildung 7: Unterhaltung über Selbstmedikation und Schulabschluss

Welche Faktoren haben nun tatsächlich einen signifikanten und eigenständigen Einfluss auf das Zustandekommen eines Gesprächs über Selbstmedikation mit dem Hausarzt? Mithilfe der multiplen logistischen Regression wurden 8 Variablen auf ihren Einfluss untersucht. Lediglich die Häufigkeit der Arztbesuche im Jahr erwies sich als Prädiktor: Patienten, die öfter als 4-mal im Jahr den Hausarzt aufsuchten, berichteten häufiger von Gesprächen über Selbstmedikation mit dem Hausarzt (Tabelle 13).

\subsection{Gründe und Themen der Unterhaltung über Selbstmedikation}

Patienten, die sich mit ihrem Hausarzt über Selbstmedikation unterhalten hatten und bei denen das Gespräch - nach eigenen Angaben - von ihnen selbst ausgegangen war $(n=48)$, nannten als Grund für die Unterhaltung vor allem Unsicherheit: Ein Viertel hatte den Arzt gefragt, ob man das betreffende Medikament ohne weiteres einnehmen könne; $20 \%$ berichteten ihrem Hausarzt von der Medikamenteneinnahme, weil sie der Meinung waren, der Arzt solle von allen Medikamenten wissen (Tabelle 14). 
Tabelle 13: Einflussfaktoren darauf, ob mit dem Hausarzt ein Gespräch über Selbstmedikation stattfindet

\begin{tabular}{|c|c|c|c|c|c|c|}
\hline \multirow[b]{2}{*}{ Variable } & \multicolumn{3}{|c|}{ unadjustiert } & \multicolumn{3}{|c|}{ adjustiert* } \\
\hline & $\mathrm{OR}^{* *}$ & $\begin{array}{c}\text { 95\%- } \\
\text { Konfidenz- } \\
\text { intervall }\end{array}$ & p & $\mathrm{OR}^{* *}$ & $\begin{array}{c}95 \%- \\
\text { Konfidenz- } \\
\text { intervall }\end{array}$ & p \\
\hline$<4$ Arztbesuche im Jahr & 1,0 & & & 1,0 & & \\
\hline$\geq 4$ Arztbesuche im Jahr & 2,5 & $1,40-4,41$ & 0,0020 & 2,5 & $1,40-4,41$ & 0,0020 \\
\hline Nicht chronisch krank & 1,0 & & & & & \\
\hline Chronisch krank ${ }^{* * *}$ & 2,7 & $1,34-5,30$ & 0,0053 & & & \\
\hline Keine Dauermedikamente & 1,0 & & & & & \\
\hline Dauermedikamente $^{* * *}$ & 2,0 & $1,14-3,52$ & 0,0158 & & & \\
\hline Männer & 1,0 & & & & & \\
\hline Frauen & 1,1 & $0,64-1,88$ & 0,7322 & & & \\
\hline Schlechte Gesundheit ${ }^{* \star * *}$ & 1,0 & & & & & \\
\hline Gute Gesundheit & 1,4 & $0,66-1,9$ & 0,6795 & & & \\
\hline Hauptschule & 1,0 & & & & & \\
\hline Realschule / Abitur & 1,3 & $0,74-2,13$ & 0,4009 & & & \\
\hline Bis 35 Jahre & 1,0 & & & & & \\
\hline Bis 55 Jahre & 1,2 & $0,52-2,50$ & 0,7704 & & & \\
\hline Ab 56 Jahre & 1,0 & $0,52-2,04$ & 0,4667 & & & \\
\hline
\end{tabular}

Variablen-Selektion durch „backward“ bei der „logistic“-Prozedur in SAS;

Level für den Verbleib der Variablen im Modell bei $p<0,05$; da keine weiteren Variablen im Modell bleiben, entsprechen sich unadjustierte und adjustierte Werte bei der einzigen verbleibenden Variablen.

** Odds Ratio.

*** Im multiplen Modell nicht mehr signifikant.

${ }^{* * * *}$ Schlechte Gesundheit wurde definiert als ein Wert von 4 und 5 auf der 5-Punkt-Likert-Skala zur Frage der selbsteingeschätzten Gesundheit. 
Tabelle 14: Grund für die Unterhaltung mit dem Hausarzt über Selbstmedikation*

\begin{tabular}{lcc}
\hline Grund (aus Patientensicht) & $\mathbf{n}$ & $\%$ \\
\hline Ist das Medikament für mich geeignet? & 10 & 25,0 \\
Kenntnis des Hausarztes erwünscht & 8 & 20,0 \\
Probleme mit dem Medikament & 6 & 15,0 \\
Angst vor Nebenwirkungen / Wechselwirkungen & 4 & 10,0 \\
Vermeidung von doppelten Verordnungen & 2 & 5,0 \\
Vertrauen (,Der Hausarzt soll von allen meinen & & 5,0 \\
Medikamenten wissen“) & 2 & 20,0 \\
Sonstiges & 8 & \\
\hline
\end{tabular}

${ }^{*} \mathrm{n}=40 ; 8$ Patienten nannten keinen Grund.

Von den 84 Patienten, die bereits über Selbstmedikation gesprochen hatten, konnten sich 63 (entspr. 100\%) an das Thema der Unterhaltung erinnern: 30,2\% sagten, es sei nur zu einem Gespräch gekommen, da der Hausarzt sich nach weiteren Medikamenten erkundigt habe; $23(36,5 \%)$ hatten sich über die Wirkung des Medikaments sowie Neben- und Wechselwirkungen unterhalten. Weitere Themen waren Rezeptfreiheit $(12,7 \%)$ sowie Wirksubstanzen und Dosierungen (je 6,4\%) (Mehrfachnennungen möglich).

Nachdem die Gründe für die Kommunikation mit dem Hausarzt über Selbstmedikation eruiert waren, wurden den Patienten zwei Meinungen vorgestellt: „Man soll mit dem Hausarzt über Selbstmedikation sprechen“ (Meinung 1) und: „Selbstmedikation ist meine eigene Sache, die geht nur mich etwas an“ (Meinung 2). 136 Personen (52,1\%) stimmten Meinung 1 und 43,3\% Meinung 2 zu. Während Frauen fast zu gleichen Teilen beiden Meinungen zuneigten, wollte die Mehrheit der Männer $(62,6 \%)$ Selbstmedikation mit dem Hausarzt besprechen. Sicherheit und Vertrauen waren wichtige Gründe für diese Ansicht; die Ungefährlichkeit von Selbstmedikation und die Möglichkeit, sich in der Apotheke beraten zu lassen, waren Gegengründe (Tabelle 15).

Vor allem Patienten unter 45 Jahren waren eher der Meinung, Selbstmedikation sei ihre eigene Sache, während ältere Patienten glaubten, es sei nötig oder sinnvoll für den Hausarzt, von der Selbstmedikation zu erfahren. Mehr Patienten mit Abitur und 
Patienten ohne Schulabschluss als Patienten mit mittlerem Bildungsstand waren der Ansicht, man solle die Selbstmedikation mit dem Hausarzt besprechen.

Tabelle 15: Gründe für oder gegen Gespräche mit dem Hausarzt über Selbstmedikation

\begin{tabular}{|c|c|c|c|c|c|}
\hline $\begin{array}{l}\text { Gründe für ein Gespräch } \\
\text { mit dem Hausarzt (Meinung 1*) }\end{array}$ & $\mathbf{n}$ & $\%$ & $\begin{array}{l}\text { Gründe gegen ein Gespräch } \\
\text { mit dem Hausarzt } \\
\text { (Meinung } 2^{* *} \text { ) }\end{array}$ & $\mathbf{n}$ & $\%$ \\
\hline Sicherheit & 34 & 26,2 & Apotheker berät & 38 & 29,9 \\
\hline Eigenes Unwissen & 32 & 24,6 & Ungefährliche Medikamente & 26 & 20,5 \\
\hline $\begin{array}{l}\text { Wechselwirkungen / } \\
\text { Nebenwirkungen }\end{array}$ & 32 & 24,6 & Zeitgründe / Bequemlichkeit & 17 & 13,4 \\
\hline $\begin{array}{l}\text { Vertrauen } \\
\text { („,der Hausarzt soll von allen } \\
\text { meinen Medikamenten wissen“) }\end{array}$ & 25 & 19,2 & Medikamente sind gut bekannt & 15 & 11,8 \\
\hline $\begin{array}{l}\text { Angst / } \\
\text { zu viele eigene Krankheiten }\end{array}$ & 3 & 2,3 & $\begin{array}{l}\text { Nicht wegen Kleinigkeiten } \\
\text { zum Hausarzt }\end{array}$ & 14 & 11,0 \\
\hline Beipackzettel nicht verständlich & 3 & 2,3 & Eigene Sache & 10 & 7,9 \\
\hline Überdosierung & 2 & 1,5 & $\begin{array}{l}\text { Seltene Einnahme } \\
\text { der Medikamente }\end{array}$ & 5 & 4,0 \\
\hline \multirow[t]{2}{*}{ Sonstiges } & 11 & 8,5 & Hausarzt hat wenig Zeit & 3 & 2,4 \\
\hline & & & Beipackzettel & 3 & 2,4 \\
\hline
\end{tabular}

(Mehrfachnennungen möglich)

* „Man soll mit dem Hausarzt über Selbstmedikation sprechen.“

** „Selbstmedikation ist meine eigene Sache, die geht nur mich etwas an.“

Das galt ebenso für Patienten mit einer chronischen Krankheit (Tabelle 16). Wohnstatus und Gesundheitszustand schienen keinen Einfluss auf die Entscheidung gehabt zu haben.

Wiederum mit Hilfe multipler logistischer Regressionen sollte - kontrolliert - untersucht werden, welche Faktoren bei Patienten die Meinung förderten, man solle die Selbstmedikation mit dem Hausarzt besprechen (Tabelle 17). Während Alter, Schulabschluss und der selbsteingeschätzte Gesundheitszustand (zum Teil differierend zu den Ergebnissen der bivariaten Analyse) keinen signifikanten Einfluss auf die Meinung hatten, war - wie bereits in den bivariaten Berechnungen - das Vorliegen einer chronischen Krankheit in hohem Maße mit der Meinung assoziiert, man solle mit dem Hausarzt über die Selbstmedikation sprechen $(O R=2,6)$. Männer vertraten ebenfalls signifikant öfter diese Meinung. Die Einnahme von Dauermedikamenten war zwar in der bivariaten Analyse signifikant mit dieser Meinung verbunden, in der multivariat kontrollierten Berechnung wirkte sich dieser Einfluss aber nicht mehr aus. Tabelle 17 stellt die ORs unadjustiert und adjustiert (multivariat kontrolliert) dar. 
Tabelle 16: Ablehnung oder Befürwortung eines Gespräches mit dem Hausarzt über Selbstmedikation*

\begin{tabular}{|c|c|c|c|}
\hline & $\begin{array}{l}\text { Für ein Gespräch mit } \\
\text { dem Hausarzt } \\
\text { (Meinung } 1^{\star *} \text { ) }\end{array}$ & $\begin{array}{l}\text { Gegen ein Gespräch } \\
\text { mit dem Hausarzt } \\
\text { (Meinung } 2^{\star * *} \text { ) }\end{array}$ & Keine Meinung \\
\hline Alter (261) & $\%$ & $\%$ & $\%$ \\
\hline $18-25$ & 37,5 & 62,5 & 0,0 \\
\hline $26-35$ & 33,3 & 58,3 & 8,3 \\
\hline $36-45$ & 50,0 & 44,2 & 5,8 \\
\hline $46-55$ & 59,0 & 41,0 & 0,0 \\
\hline $56-65$ & 56,5 & 43,5 & 0,0 \\
\hline über 65 & 62,5 & 28,1 & 9,4 \\
\hline \multicolumn{4}{|l|}{$\begin{array}{l}\text { Schulabschluss } \\
\text { (285) }\end{array}$} \\
\hline Keinen & 66,7 & 33,3 & 0,0 \\
\hline Haupt- / Volksschule & 52,7 & 43,2 & 4,1 \\
\hline Realschule & 43,8 & 50,7 & 5,8 \\
\hline Fachhochschule & 54,6 & 36,4 & 9,1 \\
\hline Abitur & 68,4 & 31,6 & 0,0 \\
\hline \multicolumn{4}{|c|}{$\begin{array}{l}\text { Chronische Krankheit } \\
\text { (261) }\end{array}$} \\
\hline $\begin{array}{l}\text { Nicht } \\
\text { krank }\end{array}$ & 34,8 & 60,6 & 4,5 \\
\hline Chronisch krank & 58,0 & 37,4 & 4,6 \\
\hline
\end{tabular}

\subsection{Empfehlung zur Selbstmedikation durch den Hausarzt aus Patientensicht}

Ein knappes Drittel der Patienten (83 / 263) berichtete, dass ihr Hausarzt ein- oder mehrmals eine Empfehlung zum Kauf eines OTC-Präparates gegeben habe. Am häufigsten geschah dies in der Altersklasse der 36- bis 45-Jährigen (39,6\%). Es gibt jedoch weder eine deutliche noch eine lineare Altersabhängigkeit. Man findet in den Altersklassen unter 35 Jahren (18-35 Jahre) seltener eine Empfehlung als in den höheren Alterklassen (Abbildung 8). 
Tabelle 17: Einflussfaktoren auf die Meinung:

„Man soll mit dem Hausarzt über Selbstmedikation sprechen“

\begin{tabular}{|c|c|c|c|c|c|c|}
\hline \multirow[b]{2}{*}{ Variable } & \multicolumn{3}{|c|}{ unadjustiert } & \multicolumn{3}{|c|}{ adjustiert* } \\
\hline & OR & $\begin{array}{c}95 \%- \\
\text { Konfidenz- } \\
\text { intervall }\end{array}$ & $\mathbf{p}$ & OR & $\begin{array}{c}\text { 95\%- } \\
\text { Konfidenz- } \\
\text { intervall }\end{array}$ & $\mathbf{p}$ \\
\hline Nicht chronisch krank & 1,0 & & & 1,0 & & \\
\hline Chronisch krank & 2,6 & $1,46-4,64$ & 0,0012 & 2,6 & $1,45-4,68$ & 0,0013 \\
\hline Frauen & 1,0 & & & 1,0 & & \\
\hline Männer & 1,9 & $1,14-3,13$ & 0,0136 & 1,9 & $1,13-3,17$ & 0,0157 \\
\hline Keine Dauermedikamente & 1,0 & & & & & \\
\hline Dauermedikamente ${ }^{* *}$ & 1,9 & $1,14-3,12$ & 0,0140 & & & \\
\hline Bis 35 Jahre & 1,0 & & & & & \\
\hline 36 bis 55 Jahre ${ }^{* *}$ & 2,1 & $1,08-4,15$ & 0,3519 & & & \\
\hline Über 55 Jahre** & 2,7 & $1,42-5,23$ & 0,0151 & & & \\
\hline Schlechte Gesundheit*** & 1,0 & & & & & \\
\hline Gute Gesundheit & 1,4 & $0,66-1,9$ & 0,6795 & & & \\
\hline$<4$ Arztbesuche im Jahr & 1,0 & & & & & \\
\hline$\geq 4$ Arztbesuche im Jahr & 1,5 & $0,88-2,38$ & 0,1463 & & & \\
\hline Realschule / Abitur & 1,0 & & & & & \\
\hline Hauptschule & 1,2 & $0,70-1,90$ & 0,5674 & & & \\
\hline
\end{tabular}

* Variablen-Selektion durch „backward“ bei der „logistic“-Prozedur in SAS;

Level für den Verbleib der Variablen im Modell bei $p<0,05$.

** Im multiplen Modell nicht mehr signifikant.

${ }^{* * *}$ Schlechte Gesundheit wurde definiert als ein Wert von 4 und 5 auf einer 5-Punkt-Likert-Skala zur Frage der selbsteingeschätzten Gesundheit.

Männer berichteten von Empfehlungen zur Selbstmedikation unabhängig vom Alter, während bei den Frauen die Empfehlungen ab einem Alter von 36 Jahren leicht zunahmen. Ein deutlicher Zusammenhang fand sich zwischen den OTC-Empfehlungen und dem Schulabschluss: Mit der Schulbildung stieg die Anzahl der Patienten, die angaben, eine Empfehlung von ihrem Hausarzt erhalten zu haben (Tabelle 18). 


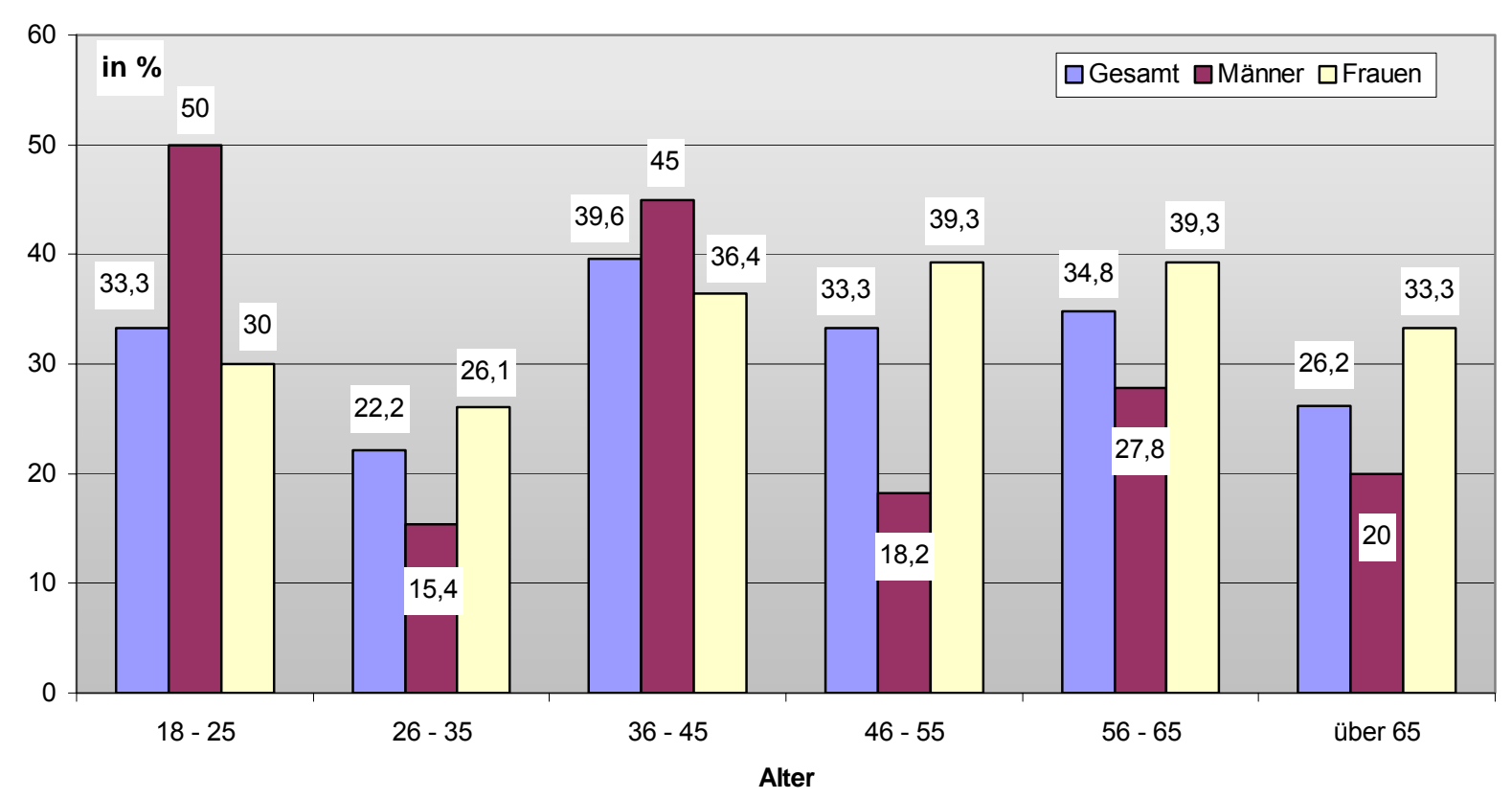

Abbildung 8: Alter der Patienten und hausärztliche Empfehlung zu Selbstmedikation

Eine Empfehlung des Hausarztes zur Selbstmedikation schien nicht von der Anzahl der chronischen Medikamente abzuhängen. Hier wurde am häufigsten eine Empfehlung an Patienten gegeben, die nur ein Medikament gegen chronische Beschwerden einnahmen, aber fast genauso häufig bei Patienten mit 5 Medikamenten. Deutlich ist jedoch, dass insgesamt häufiger Empfehlungen an Patienten mit chronischen Medikamenten ausgesprochen wurden als an diejenigen ohne Dauermedikation (Abbildung 9).

Tabelle 18: Empfehlung zur Selbstmedikation und Schulabschluss

\begin{tabular}{lrc}
\hline Schulabschluss & $\mathbf{n}$ & $\%$ \\
\hline Keinen & 1 & 11,1 \\
Haupt- / Volksschule & 41 & 27,2 \\
Realschule & 27 & 37,0 \\
Fachhochschule & 6 & 54,6 \\
Abitur & 8 & 42,1 \\
\hline
\end{tabular}


Abschließend wurde - wiederum multivariat kontrolliert - untersucht, wovon es abhängen könnte, dass Patienten - nach eigener Angabe - Selbstmedikation empfohlen wird (Tabelle 19). Von 8 untersuchten Variablen hatte nur der Schulabschluss und der selbsteingeschätzte Gesundheitszustand einen Einfluss: Je höher der Schulabschluss, desto mehr Patienten sagten, dass sie eine Empfehlung zur Selbstmedikation erhalten hatten (wie bereits bivariat angezeigt). Ebenfalls berichteten signifikant mehr Patienten, die ihre Gesundheit als eher schlecht beschrieben, über eine Empfehlung vom Hausarzt zur Selbstmedikation. Alter, Geschlecht oder das Vorliegen einer chronischen Erkrankung waren keine Prädiktoren für eine hausärztliche Empfehlung (Tabelle 19).

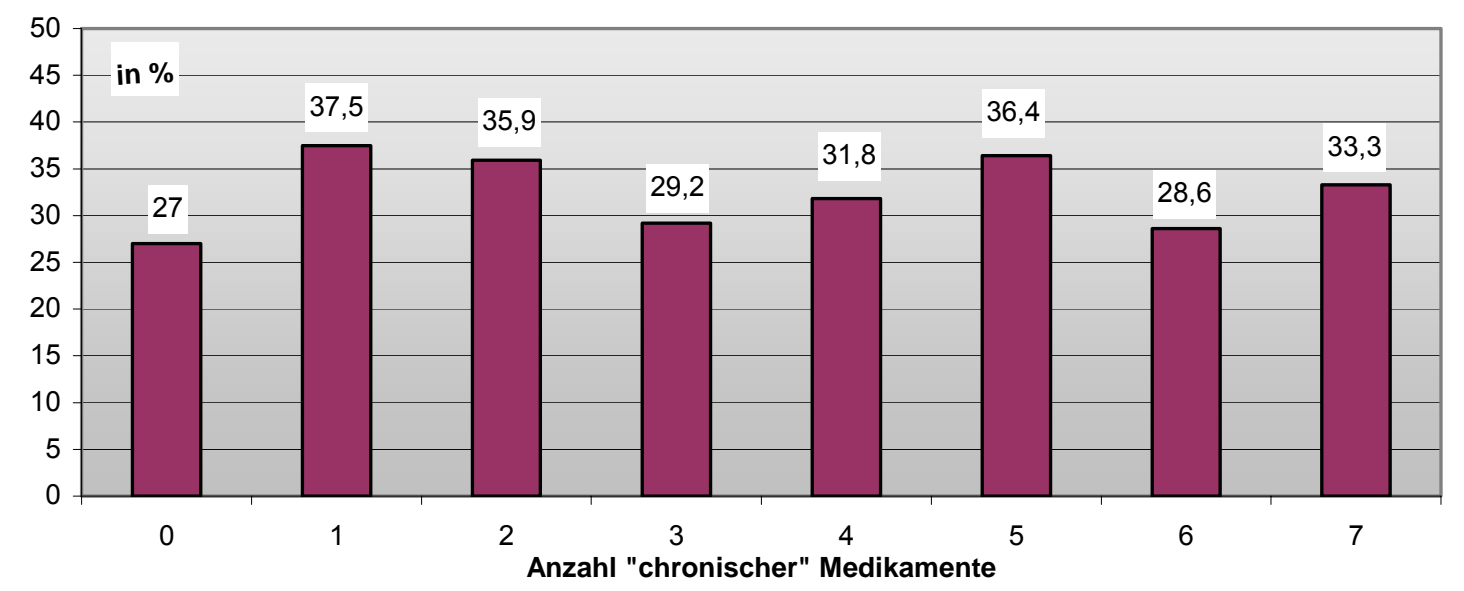
Abbildung 9: Empfehlung zur Selbstmedikation und Einnahme „chronischer“
Medikamente

\subsection{Mögliche Interaktionen zwischen Selbstmedikation und verschriebener Medikation}

Die potenzielle Gefahr von Medikamenten-Interaktionen wurde anhand der Wirkstoffe Ibuprofen und Acetylsalicylsäure (ASS) auf Seiten der Selbstmedikation und (verordneten) Betablockern, Diuretika und ACE-Hemmern antizipiert. Ibuprofen wurde 18-mal, ASS 105-mal ohne Rezept gekauft (bzw. es handelte sich um ein „Hausapotheken-Medikament"). (1) Nur in einem Fall berichteten die Befragten über die Kombination von Ibuprofen und einem Betablocker und damit potenziell die Möglichkeit einer Interaktion. Diese Kombination war dem Arzt nach Aussage des Patienten be- 
kannt. (2) Die Kombination von ASS und einem ACE-Blocker fand sich bei 8 Patienten. In 3 Fällen wusste der Hausarzt von dieser Kombination. 5 Patienten hatten das Medikament ohne Wissen des Hausarztes gekauft und eingenommen.

Tabelle 19: Einflussfaktoren auf die Empfehlung zur Selbstmedikation durch den Hausarzt

\begin{tabular}{|c|c|c|c|c|c|c|}
\hline \multirow[b]{2}{*}{ Variable } & \multicolumn{3}{|c|}{ unadjustiert } & \multicolumn{3}{|c|}{ adjustiert* } \\
\hline & OR & $\begin{array}{l}\text { 95\%-Konfidenz- } \\
\text { intervall }\end{array}$ & p & OR & $\begin{array}{l}\text { \%-Konfidenz } \\
\text { intervall }\end{array}$ & p \\
\hline Hauptschule & 1,0 & & & 1,0 & & \\
\hline Realschule / Abitur & 1,9 & $1,1-3,15$ & 0,0217 & 2,3 & $1,30-4,03$ & 0,0042 \\
\hline Gute Gesundheit & 1,0 & & & 1,0 & & \\
\hline Schlechte Gesundheit** & 1,7 & $0,97-2,88$ & 0,0659 & 2,1 & $1,19-3,83$ & 0,0114 \\
\hline$\geq 4$ Arztbesuche im Jahr & 1,0 & & & & & \\
\hline$<4$ Arztbesuche im Jahr & 1,5 & $0,86-2,56$ & 0,1587 & & & \\
\hline Männer & 1,0 & & & & & \\
\hline Frauen & 1,4 & $0,84-2,5$ & 0,1846 & & & \\
\hline
\end{tabular}

Nicht chronisch krank $\quad 1,0$

$\begin{array}{llll}\text { Chronisch krank } & 1,5 & 0,80-2,79 & 0,2087\end{array}$

Keine Dauermedikamente $\quad 1,0$

Dauermedikamente $\quad 1,4 \quad 0,82-2,45 \quad 0,2137$

Bis 35 Jahre $\quad 1,0$

$\begin{array}{llll}36 \text { bis } 55 \text { Jahre } & 1,6 & 0,79-3,29 & 0,1522 \\ \text { Über } 55 \text { Jahre } & 1,2 & 0,57-2,35 & 0,7514\end{array}$

* Variablen-Selektion durch „backward“ bei der „logistic“-Prozedur in SAS;

Level für den Verbleib der Variablen im Modell bei $p<0,05$.

${ }^{* *}$ Schlechte Gesundheit wurde definiert als ein Wert von 4 und 5 auf einer 5-Punkt-Likert-Skala zur Frage der selbsteingeschätzten Gesundheit. 


\section{7 Ärzte}

Die Datenerhebung fand, wie in Kapitel 5.1 beschrieben, in 20 Allgemeinarztpraxen statt. Insgesamt waren 58 Praxen zur Teilnahme gebeten worden (Teilnehmerrate $34,5 \%)$. In den meisten Fällen wurde kein Ablehnungsgrund genannt. Ein Arzt lehnte die Teilnahme aufgrund der erst kurz zuvor erfolgten Übernahme der Praxis ab. 5 Praxen konnte wegen Urlaubs nicht erreicht werden, eine Praxis hatte zum Zeitpunkt der Datenerhebung Urlaub. 3 Ärzte hatten zum Zeitpunkt der Datenerhebung ihre Zulassung bereits abgegeben. 5 der teilnehmenden Praxen waren Gemeinschaftspraxen mit zumeist 2, in einem Fall 3 Ärzten. Von den insgesamt 26 teilnehmenden Ärzten lehnte eine Ärztin (3,8\%) aus Zeitgründen das Interview mit mir ab. Die Interviews wurden im Gegensatz zu den Patienteninterviews in „offener Form“ geführt.

Die Hausärzte waren überwiegend Männer (20). Im Mittel waren die 26 Ärztinnen und Ärzte 49,7 Jahre alt (Min.: 39; Max: 59) und seit 14,1 Jahren niedergelassen (Min: 1; Max.: 28).

\subsection{Einschätzung der Selbstmedikation durch Hausärzte}

Die erste Frage des Arzt-Fragebogens sollte auf das Thema Selbstmedikation einstimmen. In einer Art Brainstorming konnten die Ärzte Assoziationen zur Selbstmedikation spontan äußern. Der größere Teil der Ärzte berichtete Erlebnisse und Gedanken zur Selbstmedikation. 7 Ärzte nutzen diese Einstiegsfrage, um Medikamentenklassen oder Anwendungsgebiete aufzuzählen.

Insgesamt 11 Allgemeinärzte beurteilten die Selbstmedikation vor dem Hintergrund ihrer Erfahrungen als eher positiv: 2 Ärzte wollten ihr positives Urteil auf bestimmte Mittel, hier Hausmittel und Homöopathie, beschränkt sehen. Sie verstanden Selbstmedikation als zusätzliche Medikamente, nicht als Alternative zu den verordneten Medikamenten; 2 Ärzte betonten die Eigenverantwortung des Patienten durch Selbstmedikation. Ein anderer Arzt, der die Selbstmedikation zwar als positiv einschätzte, hielt manche Patienten der Medikamentenflut "hoffnungslos ausgeliefert". Anderer Meinung war ein Arzt, der die Patienten durch den gesetzlichen Rahmen geschützt sah. 
Ein Arzt begründete seine positive Bewertung damit, dass die Patienten weniger mit Kleinigkeiten in die Sprechstunde kämen. Urteile in diese Richtung gaben auch 2 Ärzte, die gegenüber der Selbstmedikation sowohl positiv als auch negativ eingestellt waren: Kostenersparnis für den „budget-gebeutelten Arzt“, lautete ihr Argument. Beide Ärzte kamen aus einer Gemeinschafts-Dreierpraxis; 3 Ärzte brachten Selbstmedikation explizit in Zusammenhang mit der Gesundheitspolitik und / oder den Krankenkassen.

Insgesamt 6 Ärzte äußerten sich negativ: Die Einnahme zu vieler und unsinniger Medikamente sowie die mangelnde Kompetenz von Apothekern wurden als Begründungen für eine negative Beurteilung angeführt („von Apothekern abgezockt“ lautete eine Äußerung). Ein Arzt war skeptisch gegenüber der Selbstmedikation, weil ihm dadurch die Kontrolle über die Medikamenten-Einnahme entzogen sei. Anderweitig hätte er keine Einwände. Komplikationen, falsche Indikation und auch mögliche Interaktionen nannten 3 Ärzte.

8 Ärzte legten sich nicht eindeutig fest: Ein Allgemeinarzt, der weder eine positive noch negative Beurteilung zur Selbstmedikation gab, hielt das geistige Niveau des Patienten für wichtig. Dass die Patienten mit der Selbstmedikation zufriedener seien, war für einen anderen Arzt ein indirektes Kriterium der Beurteilung.

Viele der befragten Ärzte sahen ein Problem darin, dass der Hausarzt häufig nicht von der Selbstmedikation seiner Patienten wisse und dass die Patienten sie nicht in Kenntnis darüber setzten. Um mögliche Komplikationen abzuwenden, wollten 5 Ärzte gerne eine gewisse Kontrolle über die Selbstmedikation ihrer Patienten haben; 2 von ihnen würden daher die Patienten auf Selbstmedikation ansprechen. Umgekehrt berichteten 2 Ärzte, dass sie des öfteren von Patienten auf Selbstmedikation angesprochen würden - ob z.B. das eine oder andere Medikament gut für sie sei. 3 Ärzte waren der Meinung, die Patienten wüssten, was sie täten. 5 Ärzten kam im Zusammenhang mit der Selbstmedikation der Gedanke an einen Heilpraktiker. 6 Ärzte sprachen die steigende Zahl der Selbstmedikation an, wobei 4 von ihnen der Meinung waren, dass die Selbstmedikation vor allem aus Restbeständen der Medikamente bestünde, die früher verordnet wurden. 
Mehrere Ärzte sahen in der Selbstmedikation einen "Gegenspieler" zur verordneten Medikation. Oft kämen die Patienten erst, wenn die Selbstmedikation nicht gewirkt habe. Ein Problem war für 5 Ärzte die Einnahme von Medikamenten durch Familienangehörige und die Einnahme von Antibiotika, die „noch übrig waren“.

\subsection{Wichtigkeit der Kenntnis über das Selbstmedikationsverhalten der Patien- ten}

Die Frage, wie wichtig innen die Kenntnis der Selbstmedikation ihrer Patienten sei, konnten die Hausärzte auf einer 4-Punkt-Likert-Skala von „sehr wichtig“ bis „unwichtig" beantworten. Die überwiegende Anzahl, 56\% $(n=14)$, hielten diese Kenntnis für „sehr wichtig“, 28\% für „wichtig“, lediglich 2 Ärzten war es „weniger wichtig“; 2 Hausärzte gaben keine konkrete Wertung.

Gründe für die Einschätzung „sehr wichtig“ waren vor allem Vermeidung möglicher Wechsel- und Nebenwirkungen (10 Nennungen): 6 Ärzte hielten es für wichtig, von der Selbstmedikation zu wissen, um ihre eigene Therapie darauf abstimmen und beispielsweise Überdosierung und Potenzierung vermeiden zu können. Gerade darin (Überdosierung und Potenzierung) sahen 5 Ärzte eine Gefahr; 4 Ärzte beurteilten die Kenntnis von bereits eingenommener Selbstmedikation als sehr wichtig um die Krankheitszeichen richtig einschätzen zu können. Jeweils ein Arzt glaubte, durch diese Kenntnis Kontraindikationen verhindern und gegenseitiges Vertrauen aufbauen bzw. erzieherisch einwirken zu können.

Die Ärzte, die die Kenntnis über Selbstmedikation ihrer Patienten als „wichtig“ erachteten, nannten ähnliche Gründe: Jeweils 2 wollten ihre Therapie auf die bereits genommenen Medikamente abstimmen oder die Symptome besser einschätzen und deuten können. Ein Arzt sah die Gefahr von Doppelverordnungen und ein anderer nannte generell „Kontrolle“ als Grund. Ein Arzt, der es für weniger wichtig hielt, von der selbst eingenommenen Medikation seiner Patienten zu wissen, nannte als Begründung seiner Bewertung, dass Selbstmedikation sowieso „liefe“, er es aber auch jedem zutraue. 
Nur 2 Ärzte legten sich nicht fest. Ein Arzt machte seine Einschätzung vom Medikament abhängig. Er hielt aber die Kenntnis der Selbstmedikation der Patienten dann für wichtig, wenn es zu Wechselwirkungen kommen könne. Der zweite Arzt war der Meinung, dass die Patienten das Medikament sowieso bereits genommen hätten, wenn sie in der Praxis erschienen.

\subsection{Die Bereitschaft der Patienten, von ihrer Selbstmedikation zu berichten - aus Sicht der Hausärzte}

Im Sinne einer orientierenden Einschätzung bat ich die Ärzte, den Anteil der Patienten zu nennen, die trotz Nachfragen nicht von ihrer Selbstmedikation berichteten. Die offenen Antworten sind in Tabelle 20 zu Gruppen zusammengefasst.

$52 \%(n=13)$ der Hausärzte glaubten, dass der Anteil Patienten, der trotz Nachfrage ihre Selbstmedikation verschweige, bei bis zu $30 \%$ läge. 8 Hausärzte (32\%) vermuteten einen höheren Prozentsatz; 4 darunter waren der Meinung, dass 80 bis $90 \%$ ihre Selbstmedikation verschweigen würde.

Von den 4 Ärzten, die keine Angabe machten, fragten 2 die Selbstmedikationsmedikamente ihrer Patienten nicht ab, so dass sie hier keine Einschätzung geben wollten.

Tabelle 20: Anteil Patienten, die trotz Nachfrage nicht von ihrer Selbstmedikation berichten - aus Sicht der Hausärzte

\begin{tabular}{lcccccccc}
\hline \multicolumn{7}{c}{ Anteil Patienten } \\
\hline$\%$ & $0-5$ & $6-10$ & $11-20$ & $21-30$ & $31-40$ & $41-60$ & $80-90$ & Keine \\
n & 3 & 4 & 3 & 3 & 2 & 2 & 4 & 4 \\
\hline
\end{tabular}




\subsection{Einstellung des Hausarztes zur Selbstmedikation und Kommunikation mit den Patienten}

Gibt es einen Zusammenhang zwischen der Bereitschaft der Patienten, über ihre Selbstmedikation zu sprechen und der Einstellung der Hausärzte zur Selbstmedikation?

Der Anteil der Patienten, der sich schon einmal mit seinem Hausarzt über Selbstmedikation unterhalten hatte, streute in den einzelnen Praxen von 0 bis $70 \%$, im Mittel lag er bei 33,3\% (Daten aus der Patientenbefragung). Die Streuung zeigte einen gewissen Zusammenhang mit der Einstellung des Arztes zur Selbstmedikation - allerdings in anderer Richtung, als man dies erwarten würde: Insgesamt bekundeten 11 Ärzte eine positive Einstellung zur Selbstmedikation (Tabelle 21). Der Anteil Patienten, der mit diesen Ärzten über Selbstmedikation gesprochen hatte, lag im Mittel bei 19,1\% und damit sehr niedrig. Insgesamt 6 Ärzte waren mehr oder minder negativ eingestellt. Hier lag der Anteil bei 39,4\% und damit höher als bei den positiv eingestellten Ärzten. 8 Ärzte legten sich nicht fest bzw. sahen sowohl positive als auch negative Aspekte in der Selbstmedikation. Bei diesen lag der Anteil an Patienten, die über Selbstmedikation mit dem Hausarzt gesprochen hatten, bei 28,9\%.

Es zeichnete sich ein Zusammenhang ab zwischen Gesprächen über Selbstmedikation und der von den Ärzten eingeschätzten Wichtigkeit, von der Selbstmedikation ihrer Patienten zu wissen: In Praxen, in denen über ein Drittel der Patienten angab, ein Gespräch über Selbstmedikation geführt zu haben, hielten 63,6\% der Ärzte (7 / 11) es für sehr wichtig, von der Selbstmedikation der eigenen Patienten zu wissen.

Vergleicht man einzelne Aussagen der Ärzte zur Selbstmedikation aus den offenen Fragen, scheint es ebenfalls einen gewissen Zusammenhang zu den Antworten (Wahrnehmungen) der Patienten zu geben. In Praxen, in denen vergleichsweise viele Patienten von einem Gespräch mit ihrem Hausarzt über Selbstmedikation berichtet hatten, erhielt man beispielsweise folgende Aussagen (s. S. 54): 
Tabelle 21: Einstellung des Arztes zur Selbstmedikation und Kommunikation mit den Patienten

\begin{tabular}{|c|c|c|c|c|}
\hline & Praxis & $\begin{array}{l}\text { Kenntnis über die } \\
\text { Selbstmedikation der } \\
\text { Patienten }\end{array}$ & $\begin{array}{l}\text { Einstellung des Arztes } \\
\text { zur Selbstmedikation }\end{array}$ & $\begin{array}{l}\text { Patienten, die mit Hausarzt über } \\
\text { Selbstmedikation gesprochen } \\
\text { haben }\end{array}$ \\
\hline & & & & $\%$ \\
\hline & 16 & Sehr wichtig & $?$ & 70,0 \\
\hline & 18 & k.A. & + & 50,0 \\
\hline & 91 & Wichtig & $?$ & 50,0 \\
\hline & 4 & Sehr wichtig & - & 46,7 \\
\hline & 10 & Sehr wichtig & - & 43,8 \\
\hline & 14 & Sehr wichtig & - & 42,9 \\
\hline & 151 & Sehr wichtig & - & 40,0 \\
\hline & 6 & k.A. & + & 35,7 \\
\hline & 12 & Wichtig & + & 33,3 \\
\hline & 132 & Sehr wichtig & $+1-$ & 33,3 \\
\hline & 152 & Sehr wichtig & - & 33,3 \\
\hline & 1 & Sehr wichtig & - & 29,4 \\
\hline & 19 & Sehr wichtig & + & 28,6 \\
\hline & 21 & Wichtig & $+1-$ & 20,0 \\
\hline & 22 & Wichtig & $+1-$ & 20,0 \\
\hline & 133 & Sehr wichtig & $+l-$ & 20,0 \\
\hline & 17 & Weniger wichtig & + & 18,8 \\
\hline & 11 & Sehr wichtig & $?$ & 18,2 \\
\hline & 7 & Wichtig & + & 15,4 \\
\hline & 8 & Wichtig & + & 14,3 \\
\hline & 92 & Wichtig & + & 14,3 \\
\hline & 3 & Kein Arzt-Interview & & 12,5 \\
\hline & 5 & Weniger wichtig & + & 0,0 \\
\hline & 131 & Sehr wichtig & $?$ & 0,0 \\
\hline & 201 & Sehr wichtig & + & 0,0 \\
\hline & 202 & Sehr wichtig & + & 0,0 \\
\hline \multicolumn{5}{|c|}{$=$ Arzt wertete Selbstmedikation positiv } \\
\hline - & \multicolumn{4}{|c|}{$=$ Arzt wertete Selbstmedikation negativ } \\
\hline$+l-$ & \multicolumn{4}{|c|}{$=$ Arzt wertete Selbstmedikation sowohl positiv als auch negativ } \\
\hline$?$ & \multicolumn{4}{|c|}{$=$ Arzt wollte sich nicht festlegen } \\
\hline
\end{tabular}


„Ich versuche ein medizinisches Verständnis zu geben. Ich empfehle Selbstmedikation auf Privatrezept, dann haben die Patienten mehr Vertrauen" (Aussage eines Hausarztes, in dessen Praxis 50\% der Patienten von einem Gespräch mit dem Hausarzt über Selbstmedikation berichteten); „man muss Selbstmedikation immer verbinden, Vertrauen und Akzeptanz sind wichtig; lieber erklären als schimpfen; man muss die Angst nehmen, nach Selbstmedikation zu fragen" (Praxis mit $36 \%$ Gesprächsangabe); „man muss auf Selbstmedikation aufbauen“ (Praxis mit $47 \%$ Gesprächsangabe); „Selbstmedikation im Gespräch aufgreifen; ich reagiere nicht sauer“ (Praxis mit 43\% Gesprächsangabe).

\subsection{Empfehlung von Selbstmedikation durch den Hausarzt - aus Sicht der Hausärzte}

Um die Ansichten der Patienten über eine hausärztliche Empfehlung von Selbstmedikation mit denen der Ärzte vergleichen zu können, wurde den Ärzten die Frage gestellt, ob sie bei bestimmten Patientengruppen häufiger Selbstmedikation empfehlen würden (offene Frage).

Von 25 Ärzten, die am Interview teilnahmen, gaben 3 selten Empfehlungen an ihre Patienten. Ein Arzt äußerte keine Meinung. 7 Ärzte (28\%) glaubten, keine Unterschiede in der Empfehlung zu machen. Gab es Unterschiede bei der Empfehlung zur Selbstmedikation, wurde am häufigsten der „Intellekt“ der Patienten als Kriterium für oder gegen eine Medikamentenempfehlung genannt (36\%). Für jeweils 3 Ärzte spielte das Alter der Patienten bzw. die Zuverlässigkeit und die Compliance für eine Empfehlung zur Selbstmedikation eine Rolle; 2 dieser 3 Ärzte würden Selbstmedikation nicht oder seltener an Kinder und Jungendliche empfehlen. Für 8 Ärzte (32\%) war eine chronische Krankheit, vor allem Diabetes, ein Grund zur Zurückhaltung mit Empfehlungen. Schließlich hing bei 2 Ärzten die Empfehlung zur Selbstmedikation von der finanziellen Lage des Patienten ab. 


\section{Diskussion}

Hauptziel dieser Studie war es, den Bedarf von Patienten an Gesprächen über Selbstmedikation in der Hausarztpraxis aufzuzeigen. Wie schon in mehreren Studien zuvor, konnte auch hier nicht ganz geklärt werden, inwieweit das Gespräch über Selbstmedikation gewünscht wird. Es konnte jedoch deutlich gemacht werden, dass es zwei Gruppen Patienten gibt: die einen, die meinen, man solle mit dem Hausarzt über Selbstmedikation sprechen, und die anderen, die klar formulieren, es sei ihre eigene Sache.

Ein weiterer Schwerpunkt der Arbeit war die Sicht von Hausärzten auf die Kommunikation und Information über Selbstmedikation. Die Mehrzahl der befragten Ärzte stand der Selbstmedikation positiv gegenüber. Immerhin ein Viertel hatte jedoch eine negative Einstellung. Interessanterweise scheinen, nach dieser Studie, Ärzte mit eher negativer Einstellung gegenüber Selbstmedikation diese häufiger zum Thema in der Sprechstunde zu machen.

Weiterhin wurden mögliche Gefahren durch Arzneimittelinteraktionen eruiert. Diese gab es, wenn auch nur vereinzelt. Es besteht aber noch weiterer Forschungsbedarf.

\subsection{Diskussion der Methode}

Für die Datenerhebung standen 2 Möglichkeiten zur Verfügung: schriftliche oder persönliche Befragung. Aus folgenden Günden erschien die persönliche Befragung für diese Studie zweckmäßiger:

1. Es steht ein direkter Ansprechpartner für Fragen zur Verfügung (Fragen zur Studie, zur Anonymität oder Verständnisfragen).

2. Es kann eine Vertrauensbasis aufgebaut werden.

3. Die Trennung zur Praxis - und damit die Unabhängigkeit der Studie - wird deutlicher.

4. Die Antwortrate ist - im Regelfall - höher als bei einem Fragebogen (Fragebogen werden häufig nicht ausgefüllt bzw. abgegeben). 
5. Die Anzahl verwertbarer Antworten ist - im Regelfall - höher, da die Fragen weniger missverstanden werden (siehe 1.).

Vor allem ältere Patienten und Patienten in ländlichen Gegenden zeigten sich dieser Studie gegenüber eher misstrauisch. Durch das persönliche Gespräch und die Zusicherung von Vertraulichkeit konnte ich einige von innen zur Teilnahme bewegen. Fragebogen hätten bei diesen Patienten das Misstrauen sicher verstärkt. Weiterhin fällt es einigen (gerade älteren) Patienten schwer, sich schriftlich auszudrücken oder einen Fragebogen auszufüllen (Bortz und Döring 2002). Auch waren die Erinnerungslücken einiger Patienten zum Teil dadurch auszugleichen, dass ich die Patienten um Erlaubnis bitten konnte, die Medikamente bei den Arzthelferinnen zu erfragen. Von vielen Patienten wurde ich sogar hierzu gedrängt. Solche Erinnerungsprobleme bestanden bei fast jedem Patienten mit chronischen Beschwerden, so dass auf diese Weise die Validität der Datenerhebung deutlich verbessert werden konnte. In allen diesen Fällen achtete ich besonders darauf, dass mir keine persönlichen Daten über den Patienten mitgeteilt wurden und in meine Dokumentation eingingen.

Einige Fragen bezogen sich direkt auf den behandelnden Hausarzt. Für die Befragten war es daher sehr wichtig zu wissen, dass ich nicht zum Praxisteam gehörte und die Gespräche auch gegenüber dem Arzt und dem Praxisteam absolut vertraulich blieben. Gerade durch mein Auftreten (kein weißer Kittel) und eindeutige Erklärungen konnte ich die Vertraulichkeit unterstützen.

Probleme, die mit einer persönlichen Befragung einhergehen, sind folgende: Die Daten werden auf Basis der Selbstreportage erhoben. Es können also - bewusst oder unbewusst - falsche Angaben gemacht bzw. Tatsachen verschwiegen werden. Insbesondere Schamgefühle führen dazu, dass in persönlichen Befragungen wahrscheinlich häufiger sozial erwünschte Antworten gegeben werden (Bortz und Döring 2002). Weiterhin beeinflusst der Interviewer - schon durch seine Anwesenheit - indirekt und ungewollt die Antworten, und es können gegenseitige Antipathien entstehen (Bortz und Döring 2002). Schließlich ist der Zeitaufwand beim Interview höher. In der gleichen Zeit ließen sich sehr viel mehr Ergebnisse durch schriftliche Befragung erzielen. 
Die genannten Probleme wurden versucht durch folgende Maßnahmen zu minimieren: (1) Alle Interviews wurden von demselben Interviewer durchgeführt. (2) Das Thema dieser Arbeit ist kein Tabuthema und dürfte kaum die intime Privatsphäre der befragten Patienten berühren. (3) Weiterhin wurde durch die Formulierungen der Fragen versucht, Schamgefühle möglichst nicht aufkommen zu lassen. Eine Mischung aus persönlicher und patientenzugewandter Haltung einerseits und Standardisierung bei Fragen, Begrüßungstext und der Erklärung der Studie andererseits, sollte die direkte und indirekte Beeinflussung minimieren. Auf Fragen der Patienten nach der persönlichen Meinung des Interviewers wurde nicht eingegangen, aber höflich und verbindlich der Grund erklärt.

Die Antwortrate in dieser Studie ist mit fast 81\% sehr hoch (vgl. Babbie 1995; KerekBodden et al. 2000), so dass der höhere Aufwand in jedem Fall gerechtfertigt war.

\subsection{Patienten-Stichprobe}

In dieser Studie beteiligten sich mehr Frauen $(61,6 \%)$ als Männer $(38,4 \%)$. Im Vergleich mit der Gesamtbevölkerung sind die Frauen damit deutlich überrepräsentiert $(51,1 \%$ vs. $48,9 \%)$ (Statistisches Bundesamt 2004), das Verhältnis entspricht aber der im Allgemeinen häufigeren Konsultation von Ärzten durch Frauen bzw. spiegelt die höhere Lebenserwartung wider (van der Veen und Meyboom-de Jong 2004). Der Altersvergleich der befragten Patienten mit Ergebnissen des ADT-Panels des Zentralinstituts für die Kassenärztliche Versorgung (Kerek-Bodden et al. 2000) zeigt, dass die Gruppe der Patienten zwischen 50 und 59 Jahren etwas unterrepräsentiert ist (12\% vs. $16 \%)$, während die 60 - bis 69 -Jährigen über dem Vergleich liegen (22\% vs. 17\%) (Anhang 11.8, S. 102).

Durchschnittlich besuchten die Patienten dieser Studie - nach eigener Aussage - den Hausarzt 10-mal im Jahr. Dieses Ergebnis liegt etwas unter dem des ADT-Panels, in dem die Patienten im Durchschnitt 14-mal im Jahr (3,5-mal im Quartal) den Hausarzt konsultierten (Kerek-Bodden et al. 2000). Zahlen einer epidemiologischen Studie von Streich und Braun (2003) liegen mit 5,7 Besuchen (Frauen) und 4,8 Besuchen (Männer) pro Jahr unter unseren Ergebnissen. Mehr als die Hälfte der Patienten in der vorliegenden Studie gab an, 1- bis 5-mal im Jahr den Hausarzt aufzusuchen. Knapp 
$12 \%$ kamen jedoch wegen chronischer Krankheiten jeden Monat, 3,8\% sogar 2-mal im Monat, um beispielsweise Blutdruck oder Blutzucker kontrollieren zu lassen.

Der Kontakt zum aktuellen Hausarzt bestand im Mittel 11,7 Jahre und entspricht den Ergebnissen einer deutschen Telefonstudie von 2000, in der der Kontakt seit durchschnittlich 11,5 Jahren bestand (Dieterich 2000).

\section{3 Ärzte-Stichprobe}

Die Hausärzte waren im Mittel 49,7 Jahre alt (Min.: 39; Max: 59) und seit 14,1 Jahren niedergelassen (Min: 1; Max.: 28). Damit sind sie geringfügig jünger als die Allgemeinärzte in der Bundesrepublik Deutschland (50,7 Jahre) (Bundesärztekammer 2004)

\subsection{Einnahmeverhalten der Patienten}

\subsubsection{Verschriebene Medikamente}

Knapp $81 \%$ der Patienten gaben an, Medikamente einzunehmen, die innen vom Arzt verschrieben wurden, vor allem Ältere, Patienten mit niedrigerer Schulbildung und schlechter eingeschätztem Gesundheitszustand („weniger gut" und „schlecht“). Unterschiede im Geschlecht und Wohnstatus waren vernachlässigbar. Diese Ergebnisse stimmen mit einer epidemiologischen Studie aus Dänemark (Nielsen et al. 2003) überein: Auch dort nahmen Ältere signifikant häufiger Medikamente ein (45 - 66 Jahre: OR: 3,1; über 67 Jahre: OR: 9,9). Gleiches gilt für Patienten mit einer selbsteingeschätzten schlechten Gesundheit (OR: 5,8). Abweichend von vorliegender Studie verwendeten jedoch Frauen in der dänischen Untersuchung signifikant häufiger verschriebene Medikamente (OR: 1,5). 


\subsubsection{Selbstmedikation}

87,8\% der befragten Patienten nahmen Präparate zur Selbstmedikation: Frauen, Jüngere und Alleinlebende häufiger. Die Ergebnisse für Geschlecht und Wohnstatus entsprechen den Resultaten einer epidemiologischen Studie von Figueiras et al. (2000). Nielsen et al. (2003) kommen in ihrer repräsentativen Bevölkerungsstudie ebenfalls zu dem Ergebnis, dass Frauen signifikant häufiger Selbstmedikation verwenden (OR 1,8). Beide Studien kamen jedoch bezüglich des Alters zu entgegengesetzten Ergebnissen. Ebenso verhält es sich bei der epidemiologischen Studie von Sihvo et al. (2000). Sie stimmen in puncto Geschlecht mit diesen Ergebnissen überein $\left(34 \%\right.$ vs. $\left.22 \%{ }^{3}\right)$, entgegengesetzt sind jedoch ihre Ergebnisse bezüglich des Alters: Während etwas mehr als ein Viertel der Patienten zwischen 25 und 44 Jahren den Gebrauch von Selbstmedikation angaben, waren es in der Gruppe über 64 Jahren 40\%. Die Praxisstudie von Sleath et al. (2001) konnte für Erkältungs- und Allergiemedikamente zeigen, dass Frauen (OR 2,34) Selbstmedikation signifikant häufiger verwenden als Männer. Weiterhin wurde die Einnahme mit steigendem Alter signifikant seltener (OR 0,98). Auch eine Krankenhausstudie von Major et al. (1998) zeigt eine höhere Anwendung von Selbstmedikation unter Frauen (56,3\% vs. 51,4\%) und Jüngeren (57,6\% unter Patienten bis 60 Jahren vs. 48,8\% unter Patienten über 60 Jahren). Rapoport (1979) befragte 368 Patienten in Arztpraxen nach ihrem Selbstmedikationsverhalten und fand als einzige Studie, dass Männer häufiger Selbstmedikation betreiben.

Das Vorliegen eines chronischen Leidens war mit einer geringeren Einnahme von Selbstmedikation verbunden. Dies entspricht einer repräsentativen Telefonbefragung zur Selbstmedikation, in der chronisch Kranke vergleichsweise selten Selbstmedikation verwendeten (26\% vs. $40 \%$ ) (Himmel et al. 2003), während Sihvo et al. (2000) (OR 2,08), Figueiras et al. (2000) (OR 1,19) und auch Nielsen et al. (2003) (OR 1,6) jeweils multivariat kontrolliert - einen positiven Zusammenhang zwischen Langzeiterkrankung und der Einnahme von OTC-Medikamenten finden konnten. Bei allen drei Studien handelt es sich jedoch um epidemiologische Bevölkerungsstudien.

\footnotetext{
${ }^{3}$ Einnahme von OTC-Medikamenten und Vitaminen in den 2 Tagen vor der Befragung.
} 
Vor allem Schmerzmittel und Mittel gegen Erkältung kauften die Patienten in der vorliegenden Studie. Major et al. (1998) fanden ebenfalls, dass Schmerzmittel (insbesondere NSAR mit 52,7\%) die häufigsten verwendeten OTC-Medikamente sind. In ihrer Studie folgten Antibiotika, die in Deutschland der Rezeptpflicht unterliegen. An dritter Stelle standen Hypnotika und Sedativa sowie Antitussiva und Mukolytica. Im nationalen Vergleich, bezogen auf deutschlandweite Verkaufszahlen, stehen die Mittel für Erkältungsbeschwerden an erster Stelle. Erst auf dem dritten Rang folgen Schmerzmittel hinter Präparaten für Magen und Verdauung (BAH 2003; siehe oben).

\subsection{Anzahl Dauermedikamente und Bereitschaft zur Einnahme von OTC- Präparaten}

Dass Patienten, die viele Dauermedikamente nehmen, auch eher Selbstmedikation betreiben (Hypothese 3), ließ sich nicht bestätigen. Patienten, die in dieser Studie 5 und mehr Dauermedikamente einnahmen, gaben zwar am häufigsten den Gebrauch von Selbstmedikation an, boten aber mit 20 Patienten eine vergleichsweise kleine Stichprobe. Betrachtet man die Einnahme von OTC-Präparaten für Schmerz, Erkältung und Verdauung getrennt unter dieser Fragestellung, lässt sich das Ergebnis präzisieren: Während offenbar eine höhere Zahl „chronischer“ Medikamente nicht mit einer vermehrten Einnahme von Selbstmedikation bei Schmerzen und Erkältung einhergeht, nimmt Selbstmedikation bei Verdauungsproblemen mit der Anzahl an Medikamenten zu. Nach Rapoport (1979) implizieren weniger verschriebene Medikamente nicht unbedingt ein Mehr an Selbstmedikation. Major et al. (1998) fanden eine signifikant höhere Einnahme von OTC-Präparaten unter denjenigen mit weniger Medikamenten. Bei innen war der Anteil an OTC-Nutzern in der Gruppe mit 1 - 3 Medikamenten am höchsten $(58,5 \%$ vs. $41,7 \%$ unter Patienten mit mehr als 6 Medikamenten). Sie, wie auch Sihvo et al. (2000), konnten aber zeigen, dass die Einnahme von Selbstmedikation höher war unter denen, die Medikamente gegen chronische Leiden einnahmen, als unter denen, ohne jegliche Dauermedikation. Die vorliegende Studie kann auch das nicht eindeutig zeigen. 


\subsection{Selbstmedikation und Einnahme anderer Medikamente}

Schmerzmittel waren in dieser Studie häufig mit der Einnahme von Selbstmedikation verknüpft. Dies lässt sich zum einen damit erklären, dass viele Schmerzmittel als OTC-Präparate erwerbbar sind (s. oben). Interessant ist aber, dass auch Patienten, die Opioide einnahmen, zu einem hohen Anteil die Einnahme von Selbstmedikation angaben. Stimmungsaufhellende und schlaffördernde Medikamente waren ebenfalls verbunden mit hohen Selbstmedikationszahlen. Vermutlich liegt hier der gleiche Grund vor. Jedoch auch in Bereichen, in denen der Hauptteil der Medikamente der Verschreibungspflicht unterliegt (Thrombozytenaggregationshemmer, Antihypertensiva) wurde überdurchschnittlich viel (90 - 100\%) Selbstmedikation betrieben. Ein Problem gerade dieser Ergebnisse ist, dass die Stichproben zum Teil sehr klein sind. Diese Ergebnisse sind daher nur bedingt aussagefähig und müssten erst auf einer größeren Stichprobenbasis abgesichert werden.

\subsection{Einnahme von Selbstmedikation, das Krankheitsbild und die Häufigkeit der Arztbesuche}

Anschließend wurden die Beschwerden, mit denen die Patienten zum Hausarzt kamen, in Zusammenhang mit Selbstmedikation betrachtet. Patienten, die wegen Problemen des Bewegungsapparates den Hausarzt aufsuchten, verwendeten besonders häufig Präparate zur Selbstmedikation. Dies galt auch für Patienten mit Erkrankungen der Atemwege und Kreislaufbeschwerden wie KHK und Herzrhythmusstörungen. Alle Patienten, die über neurologische und psychische Probleme klagten, gaben den Gebrauch von Selbstmedikation an. Letztere Gruppe war allerdings mit nur 11 Patienten vertreten und daher nur begrenzt aussagekräftig. Vergleicht man diese Daten mit dem ADT-Panel des Zentralinstituts für die kassenärztliche Versorgung (KerekBodden et al. 2000), stellt man fest, dass die essenzielle Hypertonie und die chronisch ischämische Herzkrankheit die häufigsten Diagnosen in der Allgemeinarztpraxis darstellen, dicht gefolgt von Rückenschmerzen an dritter Stelle. Weiterhin zeigt das Panel, dass diese Diagnosen sehr häufig in Kombination auftreten, dass nämlich ein Patient mit essenzieller Hypertonie in einem Fünftel der Fälle auch an Rückenschmerzen leidet. Man könnte also postulieren, dass nicht Patienten mit essenzieller Hypertonie häufig Selbstmedikation verwenden, sondern Patienten mit Rückenbe- 
schwerden, die jedoch gleichzeitig an einer Hypertonie leiden. Dass Erkrankungen der Atemwege häufig zu Selbstmedikation führen, stimmt mit den Ergebnissen des BAH (BAH 2003) überein, die besagen, dass Husten- und Erkältungsmittel die häufigst verkauften OTC-Medikamente sind.

Zwei Studien über die Verwendung alternativer Medizin (Astin 1998, Eisenberg et al. 1998), in denen neben pflanzlichen, homöopathischen und Vitaminpräparaten auch Massagen, Selbsthilfegruppen, Relaxationstrainings u.ä. miteinbezogen wurden, berichten, dass Alternativmedizin vor allem von Patienten mit chronischen Problemen wie Rückenschmerzen, Depressionen und Kopfschmerzen angewandt wurde.

Verwenden Patienten, die seltener den Hausarzt besuchen, häufiger Selbstmedikation, wie in Hypothese 4 vermutet? Diese Frage kann durch die vorliegende Studie nicht eindeutig bejaht werden. Patienten, die weniger als 6-mal im Jahr den Hausarzt aufsuchten, berichteten in $88 \%$ über die Einnahme von Selbstmedikation während Patienten, die mehr als 2-mal im Monat zum Hausarzt gingen, in 85\% die Einnahme von Selbstmedikation angaben. Die Gruppe derer, die zwischen 13- und 24-mal im Jahr den Hausarzt aufsuchten, nahm mit $94 \%$ am häufigsten Selbstmedikation. Das Ergebnis ist also nicht eindeutig. Eine Studie von 1998 (Mclsaac et al.) untersuchte, wie viele Patienten mit Infekten der oberen Luftwege, Sinusitis, Pharyngitis und weiteren Infektionskrankheiten den Hausarzt aufsuchten: Danach war die Einnahme von OTC-Medikamenten mit weniger Besuchen beim Hausarzt wegen Erkältungskrankheiten assoziiert. Dieses Ergebnis zeigt, was mittlerweile auch viele Ärzte denken, dass nämlich Patienten sich in der Lage sehen, leichtere akute Krankheiten, wie Erkältungskrankheiten, selbst zu behandeln. Die Sichtweise niedergelassener Ärzte über Arztbesuche wegen leichter Erkrankungen zeigt eine Studie von Morris et al. (2001). Ein Großteil der Ärzte war der Meinung, dass leichte Erkrankungen zu häufig zu einem Arztbesuch führten und dass man Patienten schulen sollte, sich in solchen Fällen selbst zu helfen. Viele sahen in der Selbstmedikation eine gute Alternative. Auch 3 Ärzte dieser Studie waren der Ansicht, dass Arztbesuche wegen Bagatellerkrankungen durch Selbstmedikation minimiert würden. 


\subsection{Gespräch über Selbstmedikation mit dem Hausarzt}

Nur ein Drittel der untersuchten Patienten hatte bereits ein Gespräch über Selbstmedikation mit dem Hausarzt geführt. Damit ist die Hypothese, dass der überwiegende Teil der Patienten noch nicht mit ihrem Hausarzt über ihre Selbstmedikation gesprochen hat, bestätigt. Sleath et al. (2001) fanden dagegen, dass 58\% der von ihnen befragten Patienten, ihre Selbstmedikation mit dem Hausarzt diskutierten. Während in unserer Studie alle Patienten dazu interviewt wurden, befragten sie nur die Patienten, die zuvor angegeben hatten, Selbstmedikation zu verwenden. Stevenson et al. (2000) fanden durch Audioaufnahmen hausärztlicher Sprechstunden in Großbritannien heraus, dass in 34 von 62 Konsultationen über OTC-Medikamente gesprochen wurde. Dieses Resultat liegt ungefähr im Bereich der Studie von Sleath et al. (2001). In einer in Deutschland durchgeführten Telefonstudie von 2003 (Himmel et al.) berichteten nur $9 \%$ in den letzten 12 Monaten ein Gespräch mit dem Hausarzt über Selbstmedikation geführt zu haben. Auch wenn man die zeitliche Eingrenzung von einem Jahr im Gegensatz zu dieser Studie bedenkt, zeigen beide deutschen Studien eine deutliche Tendenz. Ein Grund für diese niedrigen Zahlen in Deutschland könnte darin liegen, dass der Hausarzt nur wenig Zeit für den einzelnen Patienten hat. Eine europäische Vergleichsstudie (van den Brink-Muinen et al. 2003) fand heraus, dass die Hausarztkonsultationen in Deutschland am kürzesten sind und deutsche Hausärzte die höchste Arbeitsbelastung haben. Auch Deveugele et al. (2002) zeigten, dass Hausarztkonsultationen in Deutschland mit durchschnittlich 7,6 Minuten die kürzesten in 6 europäischen Ländern sind. In einer Studie von Streich und Braun (2003) bewertete ein Fünftel der Patienten das aktive Informationsverhalten ihres Hausarztes (Informationen über den Gesundheitszustand, die eigene Krankheit, die medizinische Behandlung), als nur „teilweise genügend“. Jeder Achte hielt die dafür angewandte Zeit für zu kurz. Die meisten Patienten berichteten zwar, dass sie alle ihre Fragen stellen konnten, doch wenige sagten aus, der Hausarzt habe sie zu Fragen ermuntert. Cape und McCulloch (1999) befragten Patienten in Allgemeinpraxen danach, warum emotionale Probleme häufig nicht in der Arztkonsultation erwähnt werden: 48\% waren der Meinung, der Arzt habe nicht genügend Zeit, 22\% hielten ihr Problem für „zu klein“, als damit dem Arzt Zeit zu rauben, immerhin ein Viertel hatte das Gefühl, der Arzt sei nicht interessiert. Wenn der Patient kein Problem mit seinen selbst gekauften Medikamenten hat oder beim Hausarzt die Frage nach Selbstmedi- 
kation nicht zur Routine gehört, wird es in der Kürze der Zeit im Regelfall nicht zu einem Gespräch kommen. Auch die vorliegende Ärztebefragung deutet in diese Richtung. So gaben lediglich 4 von 25 Ärzten an, sich häufiger nach der Selbstmedikation ihrer Patienten zu erkundigen.

Sleath et al. (2001) befragten die Patienten, die nicht mit ihrem Hausarzt über ihre Selbstmedikation gesprochen hatten, nach dem Grund dafür. Knapp ein Viertel sagte, der Hausarzt habe sie nicht danach gefragt, und 13\% hatten es einfach vergessen. In der bereits zitierten Studie von Himmel et al. (2003) befanden fast $70 \%$ der Befragten Selbstmedikation als kein besonders wichtiges Thema in der Sprechstunde. Beachten muss man hier, dass es sich um eine epidemiologische Studie handelt und nicht nur Patienten befragt wurden. Auf die Frage nach dem Grund, das Thema Selbstmedikation anzusprechen, nannten 50\% der Patienten in der vorliegenden Studie eine konkrete Fragen zu einem bestimmten Medikament („Kann man es einnehmen“; „ich hatte Probleme mit dem Medikament“; „Angst vor Nebenwirkungen“; etc.). Nur ein Drittel informierte den Hausarzt über seine Medikamente ohne ein spezielles Problem („Hausarzt soll von allen Medikamenten wissen“; „Hausarzt soll nicht doppelt verordnen“; „Vertrauen“). Ein Drittel der Patienten gab an, dass es zu einem Gespräch über Selbstmedikation gekommen sei, weil sich der Hausarzt nach weiteren Medikamenten erkundigt habe.

Es deuten sich hier zwei differierende Erklärungen für den geringen Anteil an Gesprächen über Selbstmedikation an. Auf der einen Seite scheint es, dass Patienten kein großes Interesse an solchen Gesprächen in der Hausarztpraxis haben, auf der anderen Seite ist das Gespräch durchaus gewünscht und wird das Kommunikationsverhalten des Hausarztes kritisiert. Deutlich wird dieser Zwiespalt auch bei der Frage, ob man Selbstmedikation mit dem Hausarzt besprechen sollte oder sie die eigene Sache sei. Hier stimmten Patienten fast zu gleichen Teilen beiden Meinungen zu.

Auch mehr als die Hälfte der Ärzte in dieser Studie hielt es für „sehr wichtig“ von der Selbstmedikation der Patienten zu wissen. $28 \%$ hielten es immerhin für "wichtig“. Gründe für diese Einschätzung waren für die Ärzte vor allem Interaktionen mit verschriebener Medikation und die mögliche Verschleierung der Krankheitssymptome durch Selbstmedikation. Potenzierung durch Doppelverordnung und Abstimmen der 
Therapie waren weitere Gründe. Auch für Bradley und Bond (1995) sind dies die entscheidenden Gründe dafür, warum Hausärzte von der Selbstmedikation ihrer Patienten wissen sollten. Daher propagieren sie die Frage nach bereits eingenommenen Medikamenten als Routinefrage in der Anamnese.

Als weitere Hypothese sollte geprüft werden, ob bestimmte Patientenkollektive (Frauen, Ältere, Personen in Mehrpersonen-Haushalten und mit geringerer Schulbildung) vergleichsweise häufiger mit ihrem Hausarzt über Selbstmedikation sprechen (Hypothese 6). Vor allem Patienten aus dem Alterskollektiv der 36- bis 45-Jährigen und Patienten ab 56 Jahren sprachen über Selbstmedikation. Patienten aus Mehrpersonen-Haushalten sprachen häufiger über Selbstmedikation, nicht aber Patienten mit geringerem Schulabschluss. Ein Geschlechterunterschied war nicht festzustellen. Multivariat kontrolliert blieb jedoch nur die Anzahl der Arztbesuche im Jahr ein Prädiktor für das Zustandekommen eines Gesprächs über Selbstmedikation. Sleath et al. (1999) untersuchten das Kommunikationsverhalten über Medikamente von Ärzten und Patienten in der Sprechstunde und fanden, dass Ältere häufiger Fragen über Medikamente stellen (OR 1,02). Ein Unterschied zwischen Männern und Frauen konnten auch sie nicht feststellen. Ältere Patienten nehmen häufig mehrere Dauermedikamente ein und sind sich daher evtl. unsicher, ob es zu Interaktionen kommen kann. Unsicherheit und Angst vor Neben- und Wechselwirkungen waren in vorliegender Studie häufige Antworten auf die Frage, warum es zu einem Gespräch über Selbstmedikation kam.

Entgegengesetzt zu hier präsentierten Ergebnissen stellten bei Sleath et al. (2001) Patienten mit niedrigerem Schulabschluss häufiger Fragen über Selbstmedikation. Dass Patienten mit höherem Schulabschluss in vorliegender Studie häufiger über Selbstmedikation sprechen, könnte daran liegen, dass diese mehr Einfluss auf ihre Therapie nehmen möchten. Sie wollen besser informiert und stärker involviert sein. In einer Studie von Lupton (1997) waren Ältere und Patienten mit höherem Schulabschluss besonders an ihrer Therapie interessiert und kritischer dem Arzt gegenüber als Jüngere und Patienten mit niedrigerem Schulabschluss.

Nur 9\% der befragten Patienten, die sich - nach eigenen Angaben - noch nicht mit ihrem Hausarzt über Selbstmedikation unterhalten hatten, waren schon einmal in 
einer Situation, in der sie dies gerne getan hätten - ein deutlicher Unterschied zu der Studie von Bradley et al. (1998). Dort waren 52\% an einem Gespräch über Selbstmedikation interessiert. Möglicherweise provozierte Bradley mit der Formulierung seiner Frage einen hohen Anteil „erwünschter“ Antworten („How would you feel about your doctor enquiring about the medicines you have taken before consulting him / her?"). Die in meiner Studie weitaus indirektere Frage dürfte den tatsächlichen Informationsbedarf von Patienten angemessener widerspiegeln.

Die Autoren vermuteten, dass die Bereitschaft von Patienten, über die eigene Selbstmedikation zu sprechen, von der Haltung des Arztes zur Selbstmedikation mitgeprägt ist. Dies war der Fall - allerdings in entgegengesetzter Richtung: So wurde in Praxen, deren Ärzte eine vergleichsweise positive Meinung über Selbstmedikation hatten, zum Teil nur selten über Selbstmedikation gesprochen. Eine negative Einstellung der Ärzte hielt Patienten nicht davon ab, das Thema anzusprechen.

Weiterhin wurde deutlich, dass der Arzt dem Patienten nicht suggeriert, ein Gespräch über Selbstmedikation sei unerwünscht. Dafür spricht auch, dass die am Ende des Interviews gestellte Frage: „Gibt es bestimmte Selbstmedikation über die Sie in keinem Fall mit Ihrem Hausarzt sprechen würden?" nur von 9,5\% bejaht wurde. Eine weitere Interpretationsmöglichkeit wäre, dass die Ärzte mit einer negativen Einstellung z. B. wegen schlechter Erfahrungen mehr um die Patienten besorgt sind.

Die These, dass nach Patientenangaben das Gespräch über Selbstmedikation häufiger vom Patienten ausgeht als vom Hausarzt, konnte bestätigt werden: $60 \%$ der Patienten berichteten, das Gespräch über Selbstmedikation hätten sie initiiert. Einschränkend ist zu beachten, dass der Hausarzt diese Angaben nicht verifiziert hat bzw. aus seiner Sicht kommentierte. Dies hätte als Misstrauen und Kontrolle ausgelegt werden können. Stevenson et al. (2000) fanden hingegen, dass das Gespräch über Selbstmedikation häufiger vom Hausarzt ausging. Nur 16 von 34 Gespräche $(47,1 \%)$ über OTC-Medikamente, wurden von Patienten begonnen. An dieser Stelle ist nicht zu entscheiden, ob die Differenz in den Ergebnissen eher aus einer ungenauen Wahrnehmung der Patienten unserer Studie resultiert oder auf Unterschieden zwischen beiden Ländern beruht. So könnte z.B. der oben bereits erwähnte stärkere 
Zeitdruck in deutschen Praxen (van den Brink-Muinen et al. 2003) Ärzte vor der Frage nach Selbstmedikation zurückhalten.

\subsection{Empfehlung zur Selbstmedikation durch den Hausarzt}

Ca. 30\% aller befragten Patienten gab an, eine Empfehlung zur Selbstmedikation erhalten zu haben. Dieses Ergebnis liegt deutlich über der Studie von Bradley et al. von 1996. Hier gaben Ärzte eine Empfehlung zu einem OTC-Präparat an nur knapp $16 \%$ aller Patienten. Bradley und Mitarbeiter befragten allerdings die Ärzte nach jeder Konsultation, ob sie Selbstmedikation empfohlen hatten. Es wurden daher keine Empfehlungen erfasst, die evtl. bereits früher an den Patienten gegeben wurden.

Die vorliegenden Ergebnisse standen weder in Zusammenhang mit dem Alter der Patienten noch mit der Einnahme rezeptpflichtiger Medikamente. Pradel et al. (1999) untersuchten in ihrer Studie die Häufigkeit, mit der Ärzte Medikamente während des Patientengesprächs erwähnten. Gegenüber Frauen im Alter zwischen 16 und 34 Jahren wurden am häufigsten OTC-Medikamente erwähnt. Bei den Männern gab es keine Altersunterschiede. In der vorliegenden Studie erhielten vor allem Männer unter 45 Jahren und Frauen ab 36 Jahren Empfehlungen zur Selbstmedikation. Signifikant war der Zusammenhang einer Empfehlung zu Selbstmedikation und einem höheren Schulabschluss, sowie einem als schlecht eingeschätzten Gesundheitszustand. Dieses Ergebnis deckt sich gut mit den Aussagen der Ärzte, die häufig den "Intellekt“ als ein Kriterium für die Empfehlung zur Selbstmedikation sahen. Ein Drittel der Ärzte gab an, keine Unterschiede in der Empfehlung zu machen.

Weiterhin betrachteten Pradel et al. (1999) die Erwähnung von OTC-Medikamenten im Zusammenhang mit der Hauptdiagnose: OTC-Medikamente wurden besonders häufig bei Schwangerschaft, Diabetes mellitus, nicht-infektiöser Gastroenteritis / Colitis, akutem Infekt der oberen Luftwege und KHK genannt. Zumindest Patienten, die an Diabetes mellitus und KHK leiden, könnten als "medikamentenerfahren" bezeichnet werden. In der vorliegenden Studie wurde direkt der Zusammenhang zwischen Patienten, die eine Empfehlung zur Selbstmedikation von ihrem Hausarzt bekommen hatten, und der Anzahl an chronischen Medikamenten, die die betreffenden Personen einnahmen, untersucht. Es konnte kein Zusammenhang festgestellt werden, die 
Hypothese 11 ist abzulehnen. Deutliche Unterschiede in der Methode zwischen beiden Studie müssen jedoch berücksichtigt werden: Pradel et al. zählten direkt die Erwähnung des Medikamentes während der Sprechstunde mit Hilfe von Bandaufnahmen. Sie waren daher nicht vom Erinnerungsvermögen der Patienten abhängig. Weiterhin gingen in der zitierten Studie alle Erwähnungen eines Medikamentes in die Berechnung ein. Es wurde nicht zwischen der reinen Nennung, der Empfehlung oder einer Verschreibung unterschieden. Auch wurde die Studie bei unterschiedlichen Fachärzten durchgeführt, während diese Studie allein Hausärzte betrachtet.

\subsection{Interaktionen zwischen Selbstmedikation und verschriebener Medikation}

Mehrere zum Teil Einzelfallstudien (z.B. Johne et al. 1999; Ruschitzka et al. 2000) machen auf gefährliche Interaktionen zwischen Selbstmedikation und verschriebenen Medikamenten aufmerksam. Im Gegensatz dazu wurde in der vorliegenden Studie nach potenziellen Wechselwirkungen zwischen Medikamentenkombinationen, die in der Hausarztpraxis häufig auftreten, gesucht. Es fanden sich in 9 Fällen Kombinationen, die das Potenzial hatten, Interaktionen auszulösen. In der Hälfte der Fälle waren dem Hausarzt diese Kombinationen bekannt. Sihvo et al. (2000) zeigten, dass $4 \%$ aller befragten OTC-Nutzer Medikamentenkombinationen einnahmen, die potenziell Interaktionen auslösen konnten. In ihrer epidemiologischen Studie erfragten sie die Einnahme verschriebener und selbst erworbener Medikamente 2 Tage vor dem Interview. Es scheint folglich so zu sein, dass potenziell gefährliche Interaktionen zwischen OTC-Präparaten und verordneter Medikation in der Hausarztpraxis auftreten. Es gilt daher zu beachten, dass vor allem ältere Patienten mit vielen Dauermedikamenten von dieser Gefahr betroffen sein könnten. Dieses Ergebnis kann aber nur richtungweisend sein. Es besteht Bedarf an weiteren Studien über Medikamenteninteraktionen in der Hausarztpraxis. Studien über die klinische Relevanz potenzieller Interaktionen sollten folgen. 


\subsection{Hausärztliche Beurteilung der Selbstmedikation}

40\% der interviewten Ärzte hatten aufgrund ihrer Erfahrungen eine tendenziell positive Meinung zur Selbstmedikation. Fast ein Viertel war Selbstmedikation gegenüber eher negativ eingestellt. Die Übrigen wollten sich nicht festlegen. Studien, die konkret zu bestimmten Medikamenten fragen, kommen hier zum Teil zu positiveren Beurteilungen. So begrüßten Allgemeinärzte und Gynäkologen, in einer Studie von Sihvo et al. (1999) generell die Reklassifikation von Medikamenten in den OTC-Status. Wie aber auch in der vorliegenden Studie, war diese Einstellung medikamentenabhängig. Erwin et al. (1997) befragten britische Hausärzte über deren Einstellung zur Entlassung von H2-Antagonisten aus der Rezeptpflicht. Hier befürworteten zwar 54\% der Befragten den „Switch“, ca. ein Drittel war jedoch gegen die Entlassung. Lam und Lam (2001) befragten Hausärzte nach ihrer Meinung über die Behandlung von Infektionen der oberen Luftwege. Fast alle Befragten (95\%) plädierten für eine Schulung der Patienten im Umgang mit einfachen OTC-Präparaten, wie Paracetamol und Antihistaminika.

Gründe für eine positive Einstellung der Ärzte dieser Studie zur Selbstmedikation waren die vermutete Stärkung der Eigenverantwortung der Patienten, weniger Arztbesuche wegen sogenannter Bagatellerkrankungen und Kostenersparnis. Diese Gründe wurden ebenfalls in anderen europäischen Studien (Morris et al. 2001, Sihvo et al. 1999) als positive Aspekte der Selbstmedikation genannt. Morris et al. (2001) untersuchten die Einstellung der Hausärzte zu geringfügigen Krankheiten in der Hausarztpraxis. Hier stimmte die Mehrzahl der Ärzte darin überein, dass Selbstmedikation und größere Eigenverantwortung der Patienten die Zahl der Arztbesuche reduzieren könnte.

Gefahren der Selbstmedikation sahen die Ärzte dieser Studie in der Einnahme zu vieler und unsinniger Medikamente, durch Kontrollverlust und durch die (unterstellte) mangelnde Kompetenz der Apotheker bei der Beratung zur Selbstmedikation. Komplikationen und Wechselwirkungen waren weitere geäußerte Bedenken. Auch hier ähneln die Aussagen der Hausärzte den Aussagen von Allgemeinärzten europäischer Studien. Verlust der Kontrolle für Hausärzte, falsche Diagnose und Nebenwirkungen sind auch hier die meistgenannten Nachteile (Erwin et al. 1997; Sihvo et al. 
1999). Während in der vorliegenden Studie die Kompetenz mancher Pharmazeuten angezweifelt wurde und ein Arzt gar von „Abzockerei“ durch Apotheker sprach, hielten es die Ärzte einer Studie von Spencer und Edwards (1992) in Teilbereichen für sinnvoll, die Aufgaben der Apotheker auszuweiten, z. B. bei geringfügigen Erkrankungen zu beraten oder auch Hausärzte auf Nebenwirkungen aufmerksam zu machen. Viele Ärzte sprachen sich jedoch gegen eine Verschreibung von Medikamenten durch den Pharmazeuten aus. Fast ein Drittel war auch der Meinung, Apotheker seien zu stark durch kommerzielle Reize beeinflusst. Ein Drittel war der Ansicht, die Rolle der Pharmazeuten sollte auf das Aushändigen von Medikamenten beschränkt bleiben.

Wie hoch ist der Prozentsatz Patienten, die trotz Nachfragen nicht über ihre Selbstmedikation berichten würden? Diese Schätzfrage wurde den Ärzten gestellt, um ihre Meinung zu eruieren, ob die Frage nach Selbstmedikation überhaupt zu wahrheitsgetreuen Antworten führen würde. Überraschend viele Ärzte $(n=8)$ waren der Meinung, dass mehr als ein Drittel der Patienten ihre Selbstmedikation bei Nachfragen nicht zugeben würde. Immerhin 4 dieser Ärzte glaubten, dass mehr als $80 \%$ der Patienten auch bei Nachfrage ihre Selbstmedikation nicht angeben würde. $52 \%$ gingen davon aus, dass der Anteil 30\% nicht überschreite. Diese Angaben zeigen, dass Hausärzte zwar mehrheitlich davon ausgehen, dass das Erfragen von Selbstmedikation durchaus von den Patienten angenommen würde. Fast ein Drittel gab jedoch eher pessimistische Einschätzungen. Dem entsprechend zeigte eine dänische Krankenhausstudie von 2004 (Glintborg et al.), dass viele OTC-Medikamente häufig nicht in der Krankenakte stationärer Patienten auftauchen. Nur 54\% aller eingenommenen OTC-Medikamente waren in den Krankenakten aufgeführt. Ob allerdings diese Medikamente vom Arzt nicht abgefragt oder aber vom Patienten verschwiegen wurden, ist nicht nachvollziehbar. Patientenaussagen lassen auf eine größere Bereitschaft zu Gesprächen über Selbstmedikation schließen. Nur 9,5\% sagten, dass sie bestimmte Medikamente in keinem Fall dem Hausarzt mitteilen würden. Auch in der Literatur findet man eine positive Haltung von Patienten zu Fragen des Hausarztes zu ihrer Selbstmedikation (Bradley et al. 1998). Eine Londoner Studie (Cape und McCulloch 1999) zeigte, dass Patienten sich häufig wünschen, dass der Arzt die Initiative ergreift und Patienten zu gewissen Themen befragt. 


\subsection{Einstellung des Hausarztes zur Selbstmedikation und Kommunikation mit den Patienten}

Die Hypothese, dass in Praxen, deren Hausarzt negativ gegenüber Selbstmedikation eingestellt ist, weniger über Selbstmedikation gesprochen wird, konnte nicht angenommen werden. Es wurde in Praxen, in denen der Arzt eine negative Einstellung zur Selbstmedikation hatte, sogar geringfügig häufiger über Selbstmedikation gesprochen. Mehr Einfluss scheint die Meinung des Arztes zu haben, wie man mit Selbstmedikation umgehen sollte. Ärzte, die es für sehr wichtig hielten, die Selbstmedikation ihrer Patienten zu kennen, hatten - nach Patientenangaben - häufiger ein Gespräch über Selbstmedikation geführt. Dieses Ergebnis könnte dahingehend gedeutet werden, dass Hausärzte, die der Selbstmedikation negativ gegenüberstehen, sich möglicherweise eher der Gefahren von Selbstmedikation bewusst sind und daher häufiger die Kommunikation auf das Thema Selbstmedikation lenken. Umgekehrt werteten 4 Ärzte, die keine konkreten Angaben zur Wichtigkeit der Kenntnis über Selbstmedikation der Patienten geben wollten oder diese Kenntnis „weniger wichtig“ fanden, Selbstmedikation positiv. Dies könnte implizieren, dass Ärzte, die Selbstmedikation gutheißen, sich letztlich weniger darum kümmern und die Gefahren, die mit ihr einhergehen könnten, für nicht schwerwiegend erachten.

Auch hier wurden die Angaben der Patienten nicht verifiziert. Weiterhin wurde nicht untersucht, wann es in den einzelnen Praxen zu einem Gespräch auf Initiative des Patienten kam und wann der Arzt selber auf das Thema Selbstmedikation zu spreche kam. 


\section{Schlussfolgerungen}

Die Frage, in welchem Maße Patienten das Gespräch über Selbstmedikation wirklich wünschen, konnte nicht eindeutig geklärt werden. Die Ergebnisse dieser Studie könnte man dahingehend deuten, dass viele Patienten nicht an einem Gespräch interessiert sind. Auf der anderen Seite zeigen die Gründe für eine Unterhaltung über Selbstmedikation, dass es offenbar häufiger zu Problemen und Unsicherheit im Umgang mit Selbstmedikation kommt. Auch die Ergebnisse in der Literatur sind hier uneindeutig. Es scheint zwei Gruppen Patienten zu geben: die einen, die meinen, man solle mit dem Hausarzt über Selbstmedikation sprechen, und die anderen, die klar formulieren, es sei ihre eigene Sache. Es sieht nicht so aus, als könne man eine klare Empfehlung an Hausärzte geben, Selbstmedikation in der Sprechstunde zu thematisieren. Hier muss sich der Hausarzt individuell am Patienten orientieren. Möglicherweise ist die Form, in der nach den Ergebnissen der Studie vergleichsweise häufig die Kommunikation geführt wurde - nämlich vom Patienten induziert -, die richtige Strategie. Vielleicht sind Patienten nicht nur in der Einnahme von Medikamenten, sondern auch in der Kommunikation über diese Medikamente selbstbewusster geworden?

Unter Beachtung der schmalen Datenbasis dieser Studie scheinen potenzielle Gefahren für Interaktionen zwischen Dauermedikamenten und Selbstmedikation selten aufzutreten. Diese Ergebnisse können jedoch nur richtungweisend sein. Umfangreichere Studien zu dieser Problematik wären wünschenswert. 


\section{Zusammenfassung}

Hintergrund. Zunehmend mehr Medikamente werden in Deutschland (und vielen anderen Ländern) aus der Rezeptpflicht entlassen. Dem entspricht eine gesellschaftliche Tendenz zu größerer Patientenautonomie. Dies könnte aber umgekehrt den Wunsch und den Bedarf von Patienten / Verbrauchern nach verlässlicher Information über Medikamente verstärken.

Fragestellung. Wie groß ist der Bedarf an Gesprächen über Selbstmedikation in der Allgemeinpraxis aus Sicht von Patienten und Ärzten und wie häufig finden solche Gespräche während der Konsultation statt?

Methode. In der Grafschaft Bentheim wurden 263 Patienten (Teilnehmerrate: 80,9\%) und 26 Ärzte (Teilnehmerrate: 34,5\%) mittels standardisierter Fragebogen bzw. eines Leitfaden-Interviews zum Thema Selbstmedikation persönlich befragt.

Ergebnisse. Von 263 Patienten hatten 72\% Erfahrungen mit Selbstmedikation; $80,6 \%$ hatten in den letzten 2 Wochen vor der Befragung ein vom Arzt verschriebenes Medikament eingenommen. Frauen, Alleinlebende und Jüngere nahmen häufiger Medikamente zur Selbstmedikation. Ein Drittel (84 / 252) der Patienten hatte, laut eigener Aussage, mit dem Hausarzt über Selbstmedikation gesprochen, in $60 \%$ der Fälle auf Initiative des Patienten. Als Grund für ein Gespräch nannten Patienten vor allem Unsicherheit in der Selbstmedikation. Von den übrigen Befragten bedauerten 9\% (15/168), noch keine Gelegenheit zu einem Gespräch über Selbstmedikation gehabt zu haben. Patienten, die öfter als 4 Mal im Jahr zum Hausarzt kamen, sprachen häufiger mit ihrem Arzt über Selbstmedikation (Odds Ratio [OR]: 2,5; 95\%Konfidenzintervall: 1,4 - 4.4). Dass der Hausarzt über ihre Selbstmedikation informiert ist, wünschten signifikant häufiger Männer (OR: 1,9; 1,1 - 3,2) und chronisch Kranke (OR: 2,6; 1,5 - 4,7). Empfehlungen zur Selbstmedikation erhielten häufiger Patienten mit höherer Schulbildung (OR: 2,4; 1,3 - 4,0). Medikamentenkombinationen zwischen Selbst- und verschriebener Medikation mit potenzieller Gefahr für Interaktionen wurden in 9 Fällen gefunden. Bei 4 dieser Patienten war der Hausarzt über die Selbstmedikation informiert. Viele Ärzte (84\%) hielten es im Interview zwar für (sehr) wichtig, von der Selbstmedikation der Patienten zu wissen, wenige fragten aber regelmäßig nach Selbstmedikation.

Schlussfolgerung. Unsicherheit bei der Selbstmedikation ist für einen Teil der Patienten ein Grund für Gespräche mit dem Hausarzt. Nicht wenige aber sehen Selbst- 
medikation explizit und ausschließlich als ihre Sache. Potenziell gefährliche Kombinationen zwischen Selbstmedikation und verschriebenen Medikamenten fanden sich vergleichsweise selten und waren im Regelfall dem Hausarzt bekannt. Eine generelle Empfehlung für den Hausarzt, mit seinen Patienten über Selbstmedikation (nicht) zu sprechen, lässt sich aus diesen Ergebnissen nicht ableiten. 


\section{Literatur}

Abbott FV, Fraser MI (1998):

Use and abuse of over-the-counter analgesic agents.

J Psychiatry Neurosci $\underline{23}, 13-34$

ABDA Bundesvereinigung Deutscher Apothekerverbände, Berlin:

persönliche Mitteilung am 27.10.2003

AESGP Association of the European Self-Medication Industry (1997):

Selbstmedikation in Europa. Wo geht's lang?

Dtsch Apoth Ztg $137 / 10,31$ - 33

AESGP Association of the European Self-Medication Industry (1999):

http://www.aesgp.be/self-medication/colonne.html, 1999 (zugegriffen in 05/2003)

AESGP Association of the European Self-Medication Industry (2002):

http://www.aesgp.be/Figures02/toc02.html (zugegriffen in 05/2003)

AESGP Association of the European Self-Medication Industry (2004):

http://www.aesgp.be (zugegriffen am 27.09.2004)

Andersen M (1994):

Are $\mathrm{H}_{2}$ receptor antagonists safe over the counter drugs?

BMJ $\underline{309}, 493$ - 494

Anonym (1997):

Selbstmedikation kein Buch mit sieben Siegeln.

Medikament \& Meinung, Sept. 1997, 8

Anonym (1998):

Folgen der Zuzahlung. Weniger Arztbesuche, mehr Selbstmedikation.

Dtsch Apoth Ztg 138/17, 22 - 24

Astin JA (1998):

Why patients use alternative medicine. Results of a national study.

JAMA $\underline{279}, 1548$ - 1553 
Babbie E:

The Practice of Social Research.

7. Auflage, Wadsworth Publishing Company, Belmont 1995

BAH (1997):

Der Selbstmedikationsmarkt in der Bundesrepublik Deutschland in Zahlen, 1996.

http://www.bah-bonn.de/forum/zahlen/index.html (zugegriffen am 31.08.2004)

BAH (1998):

Der Selbstmedikationsmarkt in der Bundesrepublik Deutschland in Zahlen,1997.

http://www.bah-bonn.de/forum/zahlen/index.html (zugegriffen am 31.08.2004)

BAH (1999):

Der Selbstmedikationsmarkt in der Bundesrepublik Deutschland in Zahlen, 1998.

http://www.bah-bonn.de/forum/zahlen/index.html (zugegriffen am 31.08.2004)

BAH (2001):

Der Arzneimittelmarkt in Deutschland in Zahlen 2000 - unter besonderer Berücksichtigung der Selbstmedikation -, 2000.

http://www.bah-bonn.de/forum/zahlen/index.html (zugegriffen am 31.08.2004)

BAH (2000):

Pressemitteilung Nr. 03/2000. 1999 wieder Wachstum bei der Selbstmedikation.

http://www.bah-bonn.de/news/index.html (zugegriffen am 31.08.2004)

BAH (2002):

Der Arzneimittelmarkt in Deutschland in Zahlen 2001 - unter besonderer Berücksichtigung der Selbstmedikation -, 2001.

http://www.bah-bonn.de/forum/zahlen/index.html (zugegriffen am 31.08.2004)

BAH (2003):

Der Arzneimittelmarkt in Deutschland in Zahlen 2002 - unter besonderer Berücksichtigung der Selbstmedikation -, 2002.

http://www.bah-bonn.de/forum/zahlen/index.html (zugegriffen am 31.08.2004)

Baines DL, Whynes DK (1997):

Over-the-counter drugs and prescribing in general practice.

Br J Gen Pract $\underline{47}, 221$ - 224 
Barat I, Andreasen F, Damsgaard EMS (2000):

The consumption of drugs by 74-year-old individuals living in their own homes.

Eur J Clin Pharmacol $\underline{56}, 501$ - 509

Barat I, Andreasen F, Damsgaard EMS (2001):

Drug therapy in the elderly: what doctors believe and patients actually do.

$\mathrm{Br} \mathrm{J}$ Clin Pharmacol $\underline{51}, 615$ - 622

Berg C (1994):

Selbstmedikation: Was denkt der Verbraucher?

Pharm Ztg 139, 36 - 38

Blanc PD, Kuschner WG, Katz PP, Smith S, Yelin EH (1997):

Use of herbal products, coffee or black tea, and over-the-counter medications as self-treatments among adults with asthma.

J Allergy Clin Immunol 100, 789 - 791

Blenkinsopp A, Bradley C (1996):

Patients, society, and the increase in self medication.

BMJ $\underline{312}, 629-632$

Bortz J, Döring N:

Forschungsmethoden und Evaluation für Human- und Sozialwissenschaftler.

3. Auflage; Springer Verlag, Berlin 2002

BPI (Bundesverband der Pharmazeutischen Industrie e.V.):

Pharma-Daten 2001.

BPI Berlin, Oktober 2001

BPI (Bundesverband der Pharmazeutischen Industrie e.V.):

Pharma-Daten 2002.

BPI Berlin, Oktober 2002

BPI (Bundesverband der Pharmazeutischen Industrie e.V.):

Pharma-Daten 2003.

BPI Berlin, Oktober 2003

Bradley C, Blenkinsopp A (1996):

Over the counter drugs. The future for self medication.

BMJ $\underline{312}, 835$ - 837 
Bradley C, Bond C (1995):

Increasing the number of drugs available over the counter: arguments for and against.

Br J Gen Pract $\underline{45}, 553$ - 556

Bradley C, Kenkre J, Tobias R, Dassu D, Riaz A (1996):

Over the counter drugs. GPs' rate of recommending over the counter drugs varies.

BMJ $\underline{313}, 115-116$

Bradley C, Riaz A, Tobias RS, Kenkre JE, Dassu DY (1998):

Patient attitudes to over-the-counter drugs and possible professional responses to self-medication.

Fam Pract $\underline{15}, 44$ - 50

Bundesärztekammer (2003):

Ergebnisse der Ärztestatistik zum 31. Dezember 2003. Auswertung der statistischen Zahlen. http://www.bundesaerztekammer.de/30/Aerztestatistik/ (zugegriffen am 03.08.2004)

Bundesärztekammer (2004):

persönliche Mitteilung am 18.08.2004

Cape J, McCulloch Y (1999):

Patients' reasons for not presenting emotional problems in general practice consultations.

Br J Gen Pract $\underline{49}, 875$ - 879

Ceaser S, Wurtz R (2000):

"Leftover" antibiotics in the medicine cabinet.

Ann Intern Med $\underline{133}, 74$

Deveugele M, Derese A, van den Brink-Muinen A, Bensing J, De Maeseneer J (2002):

Consultation length in general practice: cross sectional study.

BMJ $\underline{325}, 472$ - 474

Dieterich AC:

Modelle hausärztlicher Versorgung im Meinungsbild der Bevölkerung, eine wissenschaftliche Telefonbefragung.

Med. Diss. Göttingen 2000

Doyle G, Furey S, Berlin R, Cooper S, Jayawardena S, Ashraf E, Baird L (1999):

Gastrointestinal safety and tolerance of ibuprofen at maximum over-the-counter dose.

Aliment Pharmacol Ther $\underline{13}, 897$ - 906 
Editorial Lancet (1994):

Over-the-counter drugs.

Lancet $\underline{343}, 1374-1375$

Eisenberg DM, Davis RB, Ettner SL, Appel S, Wilkey S, Van Rompay M, Kessler RC (1998):

Trends in alternative medicine use in the united states, 1990 - 1997: results of a follow-up national survey.

JAMA 2으, 1569 - 1575

EMNID-Institut:

Einfluss der hohen Patientenzuzahlung auf die Selbstmedikation.

(Im Auftrag des Bundesfachverbands der Pharmazeutischen Industrie e.V. Frankfurt/Main)

EMNID Bielefeld 1998

Ernst E (1998):

Harmless herbs? A review of the recent literature.

Am J Med 104, 170 - 178

Erwin J, Britten N, Jones R (1997):

General practitioners' views on the over-the-counter availability of $\mathrm{H}_{2}$-antagonists.

Br J Gen Pract 4ㄱ, 99 - 102

Esser B, Rehm S (1996):

Gesund ohne Arzt?

Focus $\underline{1996 / 45}, 201-210$

Figueiras A, Caamano F, Gestal-Otero JJ (2000):

Sociodemographic factors related to self-medication in Spain.

Eur J Epidemiol 16, 19 - 26

Forschungsgruppe Wahlen Online (2004):

Der OTC-Markt und das Internet.

Forschungsgruppe Wahlen Online $\mathrm{GmbH}$; Mannheim

www.forschungsgruppe.de/Aktuelles/newsletter_1.pdf (zugegriffen am 04.12.2004)

Fox JM (1997):

Zahlen zur Selbstmedikation. Analgetikamißbrauch: Risiko überschätzt?

Münch Med Wochenschr 139, 11 - 12 
Friedrich C (1990):

Selbstmedikation und Apotheke aus historischer Sicht.

Pharm Prax $\underline{45}, 73$ - 76

Glintborg B, Andersen SE, Spang-Hanssen E, Dalhoff K (2004):

The use of over-the-counter drugs among surgical and medical patients.

Eur J Clin Pharmacol $\underline{60}, 431$ - 437

Gysling E (1998):

Selbstmedikation.

Pharma-Kritik $\underline{20}, 65$ - 68

Hassell K, Noyce PR, Rogers A, Harris J, Wilkinson J (1997):

A pathway to the GP: the pharmaceutical "consultation" as a first port of call in primary health care.

Fam Pract 14, 498 - 502

Himmel W (2000):

Selbstmedikation - ökonomische, sozialpharmakologische und toxikologische Aspekte.

Dtsch Med Wochenschr $\underline{125}, 401$ - 407

Himmel W, Bardeck MA, Kochen MM (2003):

Selbstmedikation und die Rolle des Hausarztes - eine Telefonbefragung.

Z Gesundheitswiss 11, 324 - 335

Hitzenberger G (1999):

Obstipation und Laxanzien. Kosensuspapier.

Z Allgemeinmed $\underline{75}, 862$ - 866

Holden MD (1992):

Over-the-counter medications. Do you know what your patients are taking?

Postgrad Med 91, 191-4, 199 - 200

I + G Infratest und GfK Gesundheits- und Pharmamarkt-Forschung:

Selbstmedikation in der Bundesrepublik Deutschland; Ergebnisse einer Bevölkerungsumfrage.

(Im Auftrag des Bundesfachverbands der Arzneimittel-Hersteller e.V (BAH) Bonn)

I + G Infratest und GfK Gesundheits- und Pharmamarkt-Forschung, München o. J.

Johne A, Brockmöller J, Bauer S, Maurer A, Langheinrich M, Roots I (1999):

Pharmacokinetic interaction of digoxin with an herbal extract from St John's wort (Hypericum perforatum).

Clin Pharmacol Ther $\underline{66}, 338$ - 345 
Kennedy JG (1996):

Over the counter drugs. Changing the roles of doctors and pharmacists.

BMJ $\underline{312}, 593-594$

Kerek-Bodden H, Koch H, Brenner G, Flatten G (2000):

Diagnosespektrum und Behandlungsaufwand des allgemeinärztlichen Patientenklientels. Ergebnisse des ADT-Panels des Zentralinstituts für die kassenärztliche Versorgung

Z Arztl Fortbild Qualitatssich 94, 21 - 30

Knopf H, Braemer-Hauth M, Melchert HU, Thefeld W (1995):

Ergebnisse der Nationalen Untersuchungs-Surveys zum Laxanzienverbrauch.

Bundesgesundheitsblatt $1995 / 12,459$ - 467

Lam TP, Lam KF (2001):

Family doctor's attitudes towards patient self-management of upper respiratory tract infections

Hong Kong Med J ㄱ, 146 - 149

Lupton D (1997):

Consumerism, reflexivity and the medical encounter.

Soc Sci Med $\underline{45}, 373$ - 381

Major S, Badr S, Bahlawan L, Hassan G, Khogaoghlanian T, Khalil R, Melhem A, Richani R, Younes F, Yeretzian J (1998):

Drug-related hospitalization at a tertiary teaching center in Lebanon: incidence, associations, and relation to self-medicating behaviour.

Clin Pharmacol Ther $\underline{64}, 450$ - 461

Mclsaac WJ, Levin N, Goel V (1998):

Visits by adults to family physicians for the common cold.

J Fam Pract 47, 366 - 369

Miller LG (1998):

Herbal medicinals: selected clinical considerations focusing on known or potential drug-herb interactions.

Arch Intern Med 158, 2200 - 2211

Morris CJ, Cantrill JA, Weiss MC (2001):

GPs' attitudes to minor ailments.

Fam Pract 18, 581 - 585 
Myers WC, Otto TA, Harris E, Diaco D, Moreno A (1992):

Acetaminophen overdose as a suicidal gesture: a survey of adolescents' knowledge of its potential for toxity.

J Am Acad Child Adolesc Psychiatry $\underline{31}, 686$ - 690

Nielsen MW, Hansen EH, Rasmussen NK (2003):

Prescription and non-prescription medicine use in Denmark: association with socio-economic position.

Eur J Clin Pharmacol $\underline{59}, 677$ - 684

Pommer W, Bronder E, Klimpel A, Molzahn M, Helmert U, Greiser E (1989):

Regular intake of analgesic mixtures and risk of end-stage renal failure.

Lancet $\underline{1989 / 1}, 381$

Pradel FG, Hartzema AG, Mutran EJ, Hanson-Divers C (1999):

Physician over-the-counter drug prescribing patterns: an analysis of the National Ambulatory Medical Care Survey.

Ann Pharmacother $\underline{33}, 400-405$

Pschyrembel:

Klinisches Wörterbuch

259. Auflage, de Gruyter, Berlin 2002, s.v. Arzneimittel

Rapoport J (1979):

Patient's expectations and intention to self-medicate.

J R Coll Gen Pract $\underline{29}, 468$ - 472

Ruschitzka F, Meier PJ, Turina M, Lüscher TF, Noll G (2000):

Acute heart transplant rejection due to Saint John's wort.

Lancet $\underline{355}, 548$ - 549

SAS Institute Inc 1999 SAS / STAT. User's Gide Version 8.

SAS Institute, Cary, NC 1999

Schroeder K, Fahey T (2002):

Systematic review of randomised controlled trials of over the counter cough medicines for acute cough in adults.

BMJ $\underline{324}, 329$ - 331

Schwabe U:

ATC-Code. Anatomisch-therapeutisch-chemische Klassifikation für den deutschen Arzneimittelmarkt.

1. Auflage, Wissenschaftliches Institut der AOK (WidO), Bonn 1995 
Sihvo S, Hemminki E, Ahonen R (1999):

Physicians' attitudes toward reclassifying drugs as over-the-counter.

Med Care $\underline{37}, 518$ - 525

Sihvo S, Klaukka T, Martikainen J, Hemminki E (2000):

Frequency of daily over-the-counter drug use and potential clinically significant over-the-counterprescription drug interactions in the Finnish adult population.

Eur J Clin Pharmacol 트, 495 - 499

Sleath B, Roter D, Chewning B, Svarstad B (1999):

Asking questions about medication: analysis of physician-patient interactions and physician perceptions.

Med Care $\underline{37}, 1169$ - 1173

Sleath B, Rubin RH, Campbell W, Gwyther L, Clark T (2001):

Physician-patient communication about over-the-counter medications.

Soc Sci Med $\underline{53}, 357$ - 369

Smith MB, Feldman W (1993):

Over-the-counter cold medications. A critical review of clinical trials between 1950 and 1991.

JAMA $\underline{269}, 2258$ - 2263

Spencer JA, Edwards C (1992):

Pharmacy beyond the dispensary: general practitioners' views.

BMJ $\underline{304}, 1670-1672$

Statistisches Bundesamt (2004):

Statistisches Jahrbuch 2003 für die Bundesrepublik Deutschland.

http://www.destatis.de/allg/d/veroe/proser217_d.htm (zugegriffen am 15.08.04)

Stevenson FA, Barry CA, Britten N, Barber N, Bradley CP (2000):

Doctor-patient communication about drugs: the evidence for shared decision making.

Soc Sci Med $\underline{50}, 829$ - 840

Stieve G (1996):

Selbstmedikation im Spiegel von Zahlen, Trends und Meinungen.

Pharm Ztg $141 / 36,11$ - 16 
Streich W, Braun B (2003):

Versorgungsqualität in der hausärztlichen Praxis - Ergebnisse einer bundesweiten Bevölkerungsbefragung in $2001 / 2002$.

Z Allg Med $\underline{79}, 270-277$

van den Brink-Muinen A, Verhaak PF, Bensing JM, Bahrs O, Deveugele M, Gask L, Mead N, LeivaFernandez F, Perez A, Messerli V (2003):

Communication in general practice: differences between European countries.

Fam Pract $\underline{20}, 478-485$

van der Veen W, Meyboom-de Jong B:

Age and gender; in: Oxford Textbook of Primary Medical Care

hrgs. v. Jones R, Britten N, Culpepper, Gass D, Grol R, Mant D, Silagy C

Volume 1; Oxford University Press, New York 2004, 153 - 161

Walluf-Blume D (1997):

Neues Bewusstsein für die eigene Gesundheit.

Pharm Ztg 142 / 41, 30 - 34

Walluf-Blume D (1998):

Selbstmedikation: Entwicklung der EU-Märkte.

Pharm Ztg 143 / 8, 28 - 31

Walluf-Blume D (1999):

Selbstmedikation. Quo vadis?

Pharm Ind 61, 403 - 405

Wick B, Schulz M, Braun R (1998):

Selbstmedikation bei Obstipation! Beratung durch den Apotheker.

Pharm Ztg 143 / 16, 11 - 15

Winkelmann $\mathrm{HJ}$ :

Selbst hilft sich der Mann/die Frau: Tendenz zur Selbstmedikation.

in: Arzneimittel im sozialen Wandel; hrgs. v. Baier $\mathrm{H}$;

Springer Verlag, Berlin 1988, 77 - 99

WONCA International Classification Committee:

Internationale Klassifizierung der medizinischen Primärversorgung ICPC-2. Ein Codiersystem der Allgemeinmedizin.

Springer Verlag, Wien 2001 


\section{Anhang}

10.1 Patienten-Fragebogen

Fragebogen - Patienten

(Studie „Selbstmedikation“)

Nr:

Praxis:

1. Ist Dr. ..Ihr Hausarzt /lhre Hausärztin?

$\square$ Ja

$\square_{2}$ Nein

2. Seit wann ist er Ihr Hausarzt?

seit ca. Jahren

3. Wie oft im Jahr suchen Sie ihn / sie durchschnittlich auf? ca. mal / Jahr

4. Aus welchem Grund suchen Sie heute Dr. auf?

5. Waren Sie in den letzten sechs Monaten auch schon wegen anderer Beschwerden hier?

$\square$ Ja, welche $\quad \square_{2}$ Nein

6. Leiden Sie an weiteren chronischen Krankheiten,

z. B.: Zucker

Bluthochdruck

Asthma

$\square_{1}$ Ja, welche $\quad \square_{2}$ Nein
Rheuma

Magenbeschwerden

Herzschwäche 
7. Sind Sie wegen dieser Krankheiten auch bei Dr. in Behandlung?
$\square$ 1 Ja
$\square_{2}$ Nein

8. Sind Sie wegen dieser Krankheiten auch bei anderen Ärzten in Behandlung?

$\square_{1} \mathrm{Ja} \quad \square_{2}$ Nein [genauer nachfragen]

\section{Ich möchte Sie nun gerne nach einigen Medikamenten fragen}

9. Nehmen Sie im Moment Medikamente gegen Ihre aktuellen Beschwerden, also gegen. (s. Frage 4.) ein oder haben Sie deswegen bereits einmal Medikamente eingenommen?
$\square$ Ja
$\square_{2}$ Nein* $^{*}$
*weiter mit 11.

Welche davon in den letzten vier Wochen?

10. Können Sie mir die Namen dieser Medikamente nennen, bzw. [bei Erinnerungsproblemen] evtl. gegen welche Beschwerden Sie diese einnehmen?

Liste aktuelle Beschwerden [vorlesen, s. S. VII]

11. Nehmen Sie im Moment Medikamente gegen Ihre chronischen Beschwerden, also.............(s. Frage 5. u. 6.) ein oder haben Sie in den letzten vier Wochen deswegen Medikamente genommen?
$\square_{1} \mathrm{Ja}$
$\square_{2}$ Nein* $^{*}$
*weiter mit 12.

Liste chronische Beschwerden [vorlesen, s. S. VII] 
12. Nehmen Sie im Moment Medikamente gegen (Kopf)Schmerzen ein oder haben sie in den letzten vier Wochen deswegen Medikamente eingenommen?
$\square{ }_{1} \mathrm{Ja}$
$\square_{2}$ Nein* $^{*}$
*weiter mit 13.

Liste Kopfschmerzen [vorlesen, s. S. VII]

13. Nehmen Sie im Moment Medikamente gegen Verdauungsprobleme oder haben sie in den letzten vier Wochen deswegen Medikamente eingenommen?
$\square$ Ja
$\square_{2}$ Nein* $^{*}$
*weiter mit 14.

Liste Verdauungsprobleme [vorlesen, s. S. VII]

14. Nehmen Sie im Moment Medikamente gegen Erkältung oder haben sie in den letzten vier Wochen deswegen Medikamente eingenommen?
$\square$ Ja
$\square_{2}$ Nein* $^{*}$
*weiter mit 15.

\section{Liste Erkältung [vorlesen, s. S. VII]}

15. Nehmen Sie im Moment andere Medikamente oder haben sie in den letzten vier Wochen andere Medikamente eingenommen?
$\square{ }_{1} \mathrm{Ja}$
$\square_{2}$ Nein* $^{*}$
*weiter mit 16.

\section{Liste andere Medikamente [vorlesen, s. S. IX]}

16. Haben Sie sich generell schon einmal mit Ihrem Hausarzt über Medikamente, die Sie ohne Rezept gekauft haben, unterhalten?
$\square$ Ja
$\square_{2}$ Nein* $^{*}$
${ }^{*}$ weiter mit $17 \mathrm{~b}$

17a.Haben Sie inn darauf angesprochen oder hat er Sie gefragt?
$\square$ ich* $^{*}$
$\square$ er
*weiter mit 18.

17b.Gab es schon einmal eine Situation oder einen Moment, in der/dem sie sich gerne mit Ihrem Hausarzt über die Medikamente, die sie sich selber gekauft haben, unterhalten hätten? 
$\square_{1} \mathrm{Ja} \quad \square_{2}$ Nein

aus welchem Grund?

18. Was war für Sie der Grund Ihren Hausarzt deswegen anzusprechen?

Hausarzt soll alle meine Medikamente kennen

Apotheker hat mir empfohlen, mit dem Hausarzt darüber

zu sprechen

ich hatte Probleme mit dem Medikament

ich hatte Angst vor Nebenwirkungen oder Wechselwirkungen

sonstiges

19. Können Sie sich daran erinnern, um welche Themen es dabei ging?

$\square_{1} \mathrm{Ja}$, welche $\square_{2}$ Nein

20.Wir stellen immer wieder fest, dass es generell zwei Meinungen in der Bevölkerung zu der Frage gibt, ob man über die eigene Selbstmedikation mit dem Hausarzt sprechen sollte oder nicht. Die einen sind eher der Meinung, man solle mit dem Hausarzt darüber sprechen (Meinung 1), während die anderen sagen : "Nein, das ist meine eigene Sache und Verantwortung, das geht nur mich etwas an“ (Meinung 2).

Welcher Meinung würden Sie sich eher anschließen?

$\square$ Meinung $1 \quad \square$ Meinung 2 
21. Können Sie dies kurz begründen?

\section{Bei Meinung 1}

22. Gibt es trotzdem bestimmte Selbstmedikation über die Sie in keinem Fall mit Ihrem Hausarzt sprechen würden?

$\square_{1}$ Ja* $^{*} \quad \square_{2}$ Nein

*würden Sie mir diese evtl. nennen?

Mit der eigentlichen Befragung sind wir nun fertig. Darf ich Ihnen nun noch 5 Fragen für die Statistik stellen? Auch diese Fragen werden natürlich absolut anonym behandelt.

23. Wie würden Sie Ihren Gesundheitszustand im Allgemeinen beschreiben?
ausgezeichnet
sehr gut
gut
$\square$ weniger gut
$\checkmark$ schlecht

24. [Geschlecht]
$\mathrm{m}$ 
26. Welchen Schulabschluss haben Sie?

$\square$ keinen $\quad \square$ Hauptschule

$\square$ Realschule (entspr. Mittlerer Reife)

$\square$ Abitur $\quad \square$ (Fach) Hochschulabschluss

27. Leben Sie allein in Ihrem Haushalt?

$\square_{1} \mathrm{Ja}^{*} \quad \square_{2}$ Nein $\quad$ *weiter mit 28.

28. Mit wie vielen Erwachsenen und Kindern leben Sie in einem Haushalt?

29. Sind Sie von der Zuzahlung von Medikamenten befreit?
$1 \mathrm{Ja}$
$\square_{2}$ Nein

Herzlichen Dank für Ihre Mitarbeit! 


\subsection{Arzt-Fragebogen}

\section{Fragebogen - Arzt}

Praxis

( Studie „Selbstmedikation“)

1. Wenn Sie an die Patienten in Ihrer Praxis denken, was fällt Ihnen dann zu dem Stichwort „Selbstmedikation“ ein?

2. Vor diesem Hintergrund: Würden Sie die Selbstmedikation Ihrer Patienten eher als positiv oder als negativ beurteilen?
$\square$ positiv*
$\square$ negativ
* weiter mit 3.

2a. In welcher Hinsicht „negativ“? Falscher Gebrauch

Missbrauch

Fehlende Kontrolle

WWs und Interaktionen

etc.

3. Machen Sie in dieser Richtung immer wieder Erfahrungen mit Ihren Patienten? Können Sie vielleicht ein, zwei Beispiele nennen?

4. Wie wichtig ist es Ihrer Meinung nach, dass der Hausarzt von der Selbstmedikation seiner Patienten weiß?
$\square$ sehr wichtig
wichtig
weniger wichtig
unwichtig 
5. Was sind Ihre Gründe für diese Einschätzung?

6. Wie hoch schätzen Sie den Prozentsatz Ihrer Patienten, die auch bei Nachfragen nicht über ihre Selbstmedikation berichten?

7. In der Literatur findet man, dass Hausärzte Selbstmedikation nicht allen ihrer Patienten gleichermaßen empfehlen. Können Sie, im Rückblick, auch bestimmte „Patientengruppen“ benennen, denen Sie häufiger Selbstmedikation empfehlen (z.B. Männer-Frauen, Junge-Alte, chronisch Kranke,...)?

8. Was veranlasst Sie zu diesem Vorgehen?

Ich komme nun nur noch zu ein paar kurzen allgemeinen Fragen.

9. Wenn Sie an die verschiedenen Informationsquellen für die Arzneitherapie denken und deren Qualität mit den Schulnoten von 1 bis 6 beurteilen sollen, welche Noten würden Sie dann den nachfolgenden Quellen geben?

1. Fortbildungsveranstaltungen $\quad \begin{array}{lllllll}1 & 2 & 3 & 4 & 5 & 6\end{array}$

2. Kollegen

$\begin{array}{llllll}1 & 2 & 3 & 4 & 5 & 6\end{array}$

3. Pharma-Vertreter

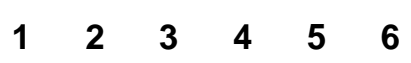

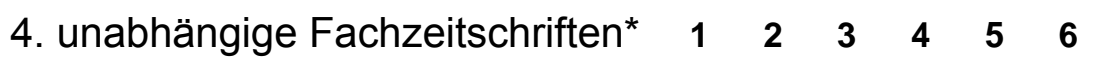

5. „übliche" Fachzeitschriften $\quad \begin{array}{llllllll} & & 2 & 3 & 4 & 5 & 6\end{array}$

* Arznei-Telegramm, Arzneimittelbrief, Pharmakritik 
10. Welche Zeitschriften schätzen Sie am meisten, wenn es um Arzneitherapie geht?

11. Wie viele Fälle rechnen Sie pro Quartal ungefähr ab:

$\square$ Unter 500

$\square 500-1000$

$\square 1000-1500$

$\square$ über 1500

12. Wie lange sind Sie als Hausarzt niedergelassen tätig?

13. Wie alt sind Sie?

14. [Geschlecht ]

Herzlichen Dank für Ihre Mitarbeit! 


\subsection{Gesamtauszählung Patienten, N (\%)}

1. Ist Dr. Ihr Hausarzt / Ihre Hausärztin? $\mathrm{Ja}$

Nein

2. Seit wann ist er Ihr Hausarzt?

3. Wie oft im Jahr suchen Sie ihn / sie durchschnittlich auf?

4. Waren Sie in den letzten sechs Monaten auch schon wegen anderer Beschwerden hier?

Ja

Nein

5. Leiden Sie an chronischen Krankheiten?

$\mathrm{Ja}$

Nein

6. Sind Sie wegen dieser Krankheiten auch bei Dr. in Behandlung? $\mathrm{Ja}$

7. Sind Sie wegen dieser Krankheiten auch bei anderen Ärzten in Behandlung? Ja Nein

8. Nehmen Sie im Moment Medikamente gegen ihre aktuellen Beschwerden oder haben sie deswegen bereits einmal Medikamente eingenommen? $\mathrm{Ja}$

Nein

10.[ Name der Medikamente ]

11. Nehmen Sie im Moment Medikamente gegen ihre chronischen Beschwerden oder haben Sie in den letzten vier Wochen deswegen Medikamente eingenommen? Ja

Nein $101 \quad(36,9)$ 
12. Nehmen Sie im Moment Medikamente gegen (Kopf)Schmerzen oder haben Sie in den letzten vier Wochen deswegen Medikamente eingenommen? $\mathrm{Ja}$

Nein

13. Nehmen Sie im Moment Medikamente gegen Verdauungsprobleme ein oder haben Sie in den letzten vier Wochen deswegen Medikamente eingenommen? $\mathrm{Ja}$

Nein

14. Nehmen Sie im Moment Medikamente gegen Erkältung oder haben Sie in den letzten vier Wochen deswegen Medikamente eingenommen? $\mathrm{Ja}$

Nein

15. Nehmen Sie im Moment andere Medikamente oder haben Sie in den letzten vier Wochen andere Medikamente eingenommen?

$\mathrm{Ja}$

Nein

16. Würden Sie eine Empfehlung zur Selbstmedikation von Ihrem Hausarzt gutheißen?

Ja

Nein

Eventuell

9

17. Haben Sie sich generell schon einmal mit lhrem Hausarzt über Medikamente, die Sie ohne Rezept gekauft haben, unterhalten? Ja

Nein

17a Haben Sie ihn darauf angesprochen oder hat er Sie gefragt?

Ich

Er

17b Gab es schon einmal eine Situation oder einen Moment, in der / dem Sie sich gerne mit ihrem Hausarzt über die Medikamente, die Sie sich selber gekauft haben, unterhalten hätten?

$\mathrm{Ja}$ 
Aus welchem Grund?

Wollte Informationen über Wechsel- und

Nebenwirkungen

Wollte Informationen über die ständige

$\begin{array}{ll}\text { Einnahme dieses Medikamentes } & 1\end{array}$

Sonstiges

$7 \quad(50,0)$

18. Was war für Sie der Grund ihren Hausarzt deswegen anzusprechen?

Kann man das Medikament einnehmen?

$11(26,8)$

Ich hatte Probleme mit dem Medikament

$6(\mathbf{1 4 , 6 )}$

Ich hatte Angst vor Wechsel- und Neben-

wirkungen

4

$(9,8)$

Der Hausarzt soll nicht doppelt verordnen

2

Vertrauen

Der Hausarzt soll von allen meinen Medi-

kamenten wissen

$8(19,5)$

Sonstiges

19. Können Sie sich daran erinnern, um welche Themen es dabei ging?

Ja

Nein

Welche?

Der Hausarzt hat sich nach den Medi-

kamenten erkundigt

Wechsel- und Nebenwirkungen

Wirksubstanzen

$4 \quad(5,9)$

Dosierung

$4 \quad(5,9)$

Rezeptfreiheit

8

$(11,8)$

Sonstiges

20. Welcher Meinung würden Sie sich eher anschließen?

0

Meinung 1

Meinung 2 
22. Gibt es trotzdem bestimmte Selbstmedikation über die Sie in keinem Fall mit Ihrem Hausarzt sprechen würden?

Ja

Nein

23. Wie würden Sie ihren Gesundheitszustand im Allgemeinen beschreiben?

Ausgezeichnet

$\begin{array}{rr}7 & (\mathbf{2}, 6) \\ 27 & (\mathbf{1 0 , 0 )} \\ 1 & (\mathbf{0 , 3 )} \\ 150 & (\mathbf{5 5}, 4) \\ 5 & (\mathbf{1}, 9) \\ 62 & (\mathbf{2 2 , 9 )} \\ 19 & (\mathbf{7}, 0)\end{array}$

24. Geschlecht

Männlich 105

Weiblich $169(\mathbf{6 1 , 7 )}$

25. Wie alt sind Sie?

Min:18;Max:87

26. Welchen Schulabschluss haben Sie?

Keinen

Haupt-(Volks)schule

Realschule 78 $(28,9)$

Abitur

(Fach)Hochschulabschluss

27. Leben Sie allein in ihrem Haushalt?

Ja

Nein

28. Mit wie vielen Erwachsenen leben Sie in einem Haushalt?

Min: 0; Max: 8

Mit wie vielen Kindern leben Sie in einem Haushalt?

Min: 0; Max: 5

28. Sind Sie von der Zuzahlung von Medikamenten befreit?

Ja

Nein

Privat versichert 
10.4 Aushang

Liebe Patientinnen und Patienten,

heute ist Frau Sajogo von der Universitätsklinik Göttingen bei uns.

Sie führt eine wissenschaftliche Studie in Hausarztpraxen durch. In diesem Zusammenhang finden Patientenbefragungen statt, zu denen vielleicht einige von Ihnen eingeladen werden.

Der Praxisablauf wird dadurch nicht gestört.

Für Sie entsteht kein Nachteil. 


\subsection{Informed consent}

Informationen zur Studie

\section{„Selbstmedikation in der Allgemeinarztpraxis“}

\section{Sehr geehrte Patientin, sehr geehrter Patient,}

die Abteilung der Allgemeinmedizin der Universität Göttingen führt eine Studie bei Hausärzten durch. Wir möchten im Rahmen einer Doktorarbeit erfahren, wie Patienten und Patientinnen das Angebot der Selbstmedikation, also den Kauf von Medikamenten ohne Rezept, nutzen. Selbstmedikation meint die eigenverantwortliche Einnahme von nicht-verschreibungspflichtigen Arzneimitteln ohne vorausgegangene ärztliche Verordnung. Diese kann mit oder ohne beratende Unterstützung durch einen Apotheker oder anderen Heilberufler erfolgen.

\section{Dazu brauchen wir Ihre Hilfe!}

Wir haben Sie, wie auch andere Patienten dieser Praxis, per Zufall ausgewählt und bitten Sie, uns einige Fragen zu beantworten. Selbstverständlich gilt die ärztliche Schweigepflicht. Ihre Antworten werden vertraulich behandelt und anonym ausgewertet. Wenn Sie an der Befragung nicht teilnehmen wollen, wird dies weder Ihr Arzt erfahren noch werden Sie irgendwelche Nachteile haben.

Durch Ihre Mitarbeit können Sie dazu beitragen, dass die Wünsche und Bedürfnisse von Patienten in Erfahrung gebracht und damit besser in die ärztliche Praxis umgesetzt werden können!

Vielen Dank für Ihre Aufmerksamkeit

Ines Sajogo

\section{Betreuung:}

Die Informationen zur Studie haben Sie von mir (Ines Sajogo, Studierende der Universität Göttingen) erhalten. Sollten Sie noch Fragen haben, so wenden Sie sich doch bitte an mich.

Verantwortlich für diese Studie ist:

Priv.-Doz. Dr. Wolfgang Himmel (Abteilung Allgemeinmedizin) 


\subsection{Arzneimittelgesetz}

§ 49 Abs.4 Nr. 3 Arzneimittelgesetz

„Das Bundesministerium wird ermächtigt, durch Rechtsverordnung, die nicht der Zustimmung des Bundesrates bedarf, die Verschreibungspflicht aufzuheben, wenn nach Nummer 1 auf Grund der bei der Anwendung des Arzneimittels gemachten Erfahrungen feststeht, dass die Voraussetzungen des $\S 48$ Abs. 2 Nr. 1 nicht vorliegen."

§ 48 Abs. 2 Nr.1 Arzneimittelgesetz

„Das Bundesministerium wird ermächtigt, im Einvernehmen mit dem Bundesministerium für Wirtschaft nach Anhörung von Sachverständigen durch Rechtsverordnung mit Zustimmung des Bundesrates Stoffe, Zubereitungen aus Stoffen oder Gegenstände zu bestimmen,

a) die die Gesundheit des Menschen (...) oder der Umwelt auch bei bestimmungsgemäßem Gebrauch unmittelbar oder mittelbar gefährden können, wenn sie ohne ärztliche, zahnärztliche (...) Überwachung angewendet werden, oder

b) die häufig in erheblichem Umfange nicht bestimmungsgemäß gebraucht werden, wenn dadurch die Gesundheit von Mensch (...) unmittelbar oder mittelbar gefährdet werden kann.“ 
10.7 Aus der Rezeptpflicht entlassene Medikamente (Stand: 2003)

\begin{tabular}{l|l}
\hline Medikament & Medikament \\
\hline Ibuprofen & Beclometason \\
Oxiconazol & Acelastin \\
Astemizol & Famotidin \\
Naftifin & Ranitidin \\
Ketoconazol & Diclofenac \\
Tioconazol & Nedocromil \\
Aciclovir & D-Glucosamin \\
Selen-(IV)-sulfid & Pirenoxin \\
Loperamid & Levocabastin \\
Nicotin & Croconazol \\
Acetylcystein & Amorolfin \\
Clotrimazol & Sertoconazol \\
Etofenamat & Terbinafin \\
Piroxicam & Lufenuron \\
Loratadin & Naproxen \\
Lactitol & Lodoxamid \\
Felbinac & Icodextrin \\
Flufenaminosäure & Permethrin \\
Dimethysulfoxid & Triamcinolon \\
Citirizin & \\
Indometazin & \\
Hydrocortison & \\
Miconazol & \\
Ibuprofen & \\
Fenticonazol & \\
\hline
\end{tabular}

(nach ABDA 2003, nach Walluf-Blume 1999) 
10.8 Altersverteilung der teilnehmenden Patienten

\begin{tabular}{lcc}
\hline $\begin{array}{l}\text { Alter } \\
\text { in Jahren }\end{array}$ & $\begin{array}{c}\text { Eigene Studie }(\mathbf{n}=\mathbf{2 5 7}) \\
\%\end{array}$ & $\begin{array}{c}\text { ADT-Panel }(\mathbf{n}=\mathbf{6 1 2 4 0})^{*} \\
\text { 20-29 }\end{array}$ \\
$30-39$ & 12,1 & 13,0 \\
$40-49$ & 18,7 & 18,9 \\
$50-59$ & 17,1 & 15,4 \\
$60-69$ & 12,1 & 16,1 \\
über 69 & 22,2 & 17,1 \\
\hline
\end{tabular}

* Kerek-Bodden et al. 2000 


\section{Danksagung}

Ich danke Herrn Priv.-Doz. Dr. Wolfgang Himmel für die freundliche Überlassung des Themas der Dissertation sowie die hervorragende Betreuung und viele wichtige Anregungen.

Herrn Prof. Dr. Michael M. Kochen, MPH, FRCGP danke ich für die konstruktive Kritik. Weiterhin danke ich den Mitarbeitern der Abteilung Allgemeinmedizin der Universität Göttingen für die stets kollegiale Unterstützung.

Mein besonderer Dank gilt den Ärzten und Patienten, die sich bereit erklärt haben, an der Studie teilzunehmen. 


\section{Lebenslauf}

Am 14. Mai 1978 wurde ich als zweites von vier Kindern von Beatrix und Dr. med. Subagio Sajogo in Haltern geboren. Von 1984 bis 1988 besuchte ich die Grundschule zu Nordhorn. Nach zwei Jahren an der Freiherr-vom-Stein-Orientierungsstufe wechselte ich 1990 zum Gymnasium Nordhorn, wo ich 1997 mein Zeugnis über die Hochschulreife erhielt.

Von 1997 bis 2004 studierte ich Humanmedizin an der Georg-August-Universität zu Göttingen, unterbrochen von einem Semester an der Universität Basel, Schweiz. Im Rahmen des Praktischen Jahres leistete ich ein halbes Tertial im Mercy Hospital for Women in Melbourne, Australien ab sowie ein Tertial in der Klinik Gut in St. Moritz, Schweiz.

Am 11. Mai 2004 bestand ich die Ärztliche Prüfung und erhielt im Oktober die Approbation zur Ärztin. Seit dem 1. Januar 2005 arbeite ich als Assistenzärztin in der Frauenklinik des Marienhospitals zu Osnabrück. 


\section{Abstract}

Background. In Germany and many other countries, an increasing number of drugs switch into non-prescription-status and are available over the counter (OTC). This development corresponds a social tendency to more patient autonomy, but could also increase the need for reliable information. Aim of study. To examine (1) the need for communication about self medication in general practice consultations, seen from the patient's and the doctor's view, and (2) the reasons as well as the initiative for conversations about self medication. Methods. Data were collected from 26 general practitioners (GPs) and 263 patients surveyed on basis of standardized questionnaires. Participation rate was $80,9 \%$ in patients and $34,5 \%$ in doctors. Results. $72 \%$ (189 / 263) of the patients reported to have used self medication products; $80,6 \%$ had used prescription only medicines (POMs) during the last four weeks prior to the interview. Women, singles, younger patients were more likely to use OTCproducts. One-third (84 / 252) remembered a conversation with their GP about self medication - more than half $(60 \%)$ initiated by the patients themselves. Uncertainty was the most often cited reason for these conversations. Of those who never talked about self medication with their GP, only $9 \%(15 / 168)$ would like to do so. Patients who visited their GP more often than four times a year were more likely to talk with their doctor about self medication (Odds Ratio [OR]: 2,5; 95\%-confidence interval: 1,4 - 4,4). Significantly more men (OR: 1,9; $1,1-3,2$ ) and chronically ill patients (OR: 2,6; $1,5-4,7$ ) wanted their GP to be informed about their self medication. Doctors' recommendations for self medication were more commonly given to higher educated patients (OR: 2,4; 1,3 - 4,0). Drug-combinations with potential dangerous interactions between OTC-drugs and POMs were found in nine cases. In four of these cases the GP was informed about the drugs. Although $84 \%$ of the GPs considered it (very) important to know about their patients' self medication, only a few asked regularly about it. Conclusions. Uncertainty in drug-taking is the strongest motive for patients to talk about self medication with their GP. However, only few patients feel a strong need for conversations about self medication and many patients consider self medication their private affair. In any case, dangerous combinations between OTC-drugs and POMs seem to be a rare event. The results of our study do not justify a general proposal for GPs to ask - or not to ask - patients about their self medication. 